\title{
Modelos de regressão beta e simplex para análise de proporções
}

\author{
Eliane Shizue Miyashiro
}

DissertaÇÃo APRESENTADA

AO

Instituto de Matemática e Estatística

DA

Universidade De SÃo Paulo

PARA

OBTENÇÃO DO TÍTULO

DE

Mestre em CiÊnCias

Área de Concentração: Estatística

Orientadora: Profa. Dra. Denise Aparecida Botter

São Paulo, abril de 2008. 


\title{
Modelos de regressão beta e simplex para análise de proporções
}

\author{
Este exemplar corresponde à redação final da \\ dissertação de mestrado devidamente corrigida \\ e defendida por Eliane Shizue Miyashiro \\ e aprovada pela comissão julgadora.
}

São Paulo, 24 de abril de 2008.

Banca Examinadora:

- Profa. Dra Denise Aparecida Botter (orientadora) - IME - USP

- Prof. Dr. Rinaldo Artes - IBMEC - SP

- Prof. Dr. Gauss Moutinho Cordeiro - UFRPE 


\section{Agradecimentos}

Durante esses anos de estudo, contei com a ajuda de muitas pessoas, entre as quais expresso minha imensa gratidão:

Às professoras Denise Aparecida Botter e Mônica Carneiro Sandoval, pela orientação, paciência e confiança dedicadas a mim na elaboração desta dissertação.

Aos meus pais Nelson e Marie, pelo amor e educação que sempre me ofereceram.

Ao meu irmão Ivan, pelo carinho e apoio que me acompanha desde a infância.

Aos demais familiares, que sempre me deram carinho, me apoiaram e entenderam minha ausência em determinados momentos.

Aos amigos de infância, que de perto ou de longe sempre estiveram ao meu lado.

Aos amigos do mestrado, em especial às amigas que estudaram comigo desde a graduação no IME-USP, pela amizade e incentivo sem os quais não teria enfrentado os momentos difíceis de estudo.

Aos amigos do trabalho, que sempre me incentivaram e compreenderam meu horário dividido com os estudos.

Aos demais amigos, que sempre me apoiaram.

Aos participantes da banca examinadora, pelas valiosas contribuições. 


\section{Resumo}

Diversos estudos compreendem a análise de variáveis definidas no intervalo $(0,1)$, como porcentagens ou proporções. Os modelos mais adequados são os de regressão baseados nas distribuições beta e simplex. Neste trabalho, apresentamos o modelo de regressão beta proposto por Ferrari \& Cribari-Neto (2004) e desenvolvemos o modelo de regressão simplex. Definimos um resíduo para o modelo de regressão simplex, muito útil na análise de diagnóstico, a partir do trabalho de Espinheira, Ferrari \& Cribari-Neto (2008). Apresentamos uma forma geral para algumas medidas de diagnóstico, que podem ser aplicadas para os dois modelos. Avaliamos os modelos de regressão beta e simplex por meio de duas aplicações a dados reais, utilizando essas medidas. 


\section{Abstract}

Many studies consider the analysis of variables restricted to the interval $(0,1)$, as percentages and proportions. The most recommended models are based upon the beta and simplex distributions. In this work, we present the beta regression model proposed by Ferrari and Cribari-Neto (2004) and develop the simplex regression model. We propose a residual for the simplex regression model, which is very useful for the diagnostic analysis, based upon the work of Espinheira et al. (2008). We generalize some diagnostic techniques that can be applied to both models. We evaluate the beta and simplex models by two applications to real data, using those techniques. 


\section{Sumário}

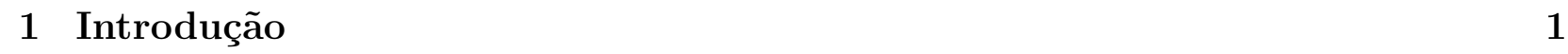

1.1 Organização da Dissertação $\ldots$. . . . . . . . . . . . . . . . . . . . . . . 2

1.2 Suporte Computacional . . . . . . . . . . . . . . . . . . . 3

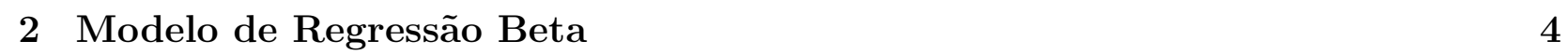

2.1 Introdução . . . . . . . . . . . . . . . . . . . . . . . . 4

2.2 Distribuição Beta . . . . . . . . . . . . . . . . . . . . . . . 4

2.3 Definição do Modelo de Regressão Beta . . . . . . . . . . . . . . . . . . . . . . 5

$2.3 .1 \quad$ Função Escore e Matriz de Informação de Fisher . . . . . . . . . . . . . 8

2.3 .2 Estimação dos parâmetros . . . . . . . . . . . . . . . . . . . 10

2.4 Intervalos de Confiança . . . . . . . . . . . . . . . . . . . . . . . . . 11

2.5 Resíduos . . . . . . . . . . . . . . . . . . . . . . . . . . . . . 12

2.5.1 Resíduo ordinário . . . . . . . . . . . . . . . . . . . . . 12

2.5 .2 Resíduo ponderado padronizado 1 . . . . . . . . . . . . . . . . 12

2.5 .3 Resíduo ponderado padronizado 2 . . . . . . . . . . . . . . 13

3 Modelo de Regressão Simplex 15

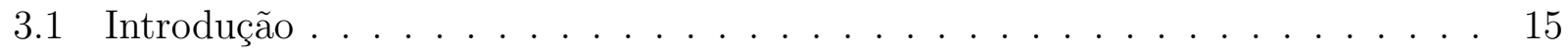

3.2 Distribuição Simplex . . . . . . . . . . . . . . . . . . . . . . . . . . . . 15 
3.3 Definição do Modelo de Regressão Simplex . . . . . . . . . . . . . . . . . . . . 16

$3.3 .1 \quad$ Função Escore e Matriz de Informação de Fisher . . . . . . . . . . . . . 16

$3.3 .2 \quad$ Estimação dos parâmetros . . . . . . . . . . . . . . . . . . . . . . . 20

3.4 Resíduos . . . . . . . . . . . . . . . . . . . . . . . . . . . . . . . . 21

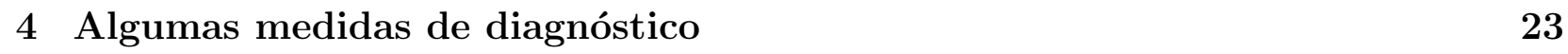

4.1 Introdução . . . . . . . . . . . . . . . . . . . . . . . . . . . 23

4.2 Ponto Alavanca . . . . . . . . . . . . . . . . . . . . . . . . . . . . . . . 24

4.3 Ponto Aberrante . . . . . . . . . . . . . . . . . . . . . . . . . . . . 25

4.4 Distância de Cook . . . . . . . . . . . . . . . . . . . . . . . . . . . . 25

4.5 Critério de Informação de Akaike (AIC) $\ldots$. . . . . . . . . . . . . . . . 26

4.6 Gráfico de Probabilidade Meio-Normal com Envelope Simulado. . . . . . . . . 26

$\begin{array}{llr}5 & \text { Aplicações } & 28\end{array}$

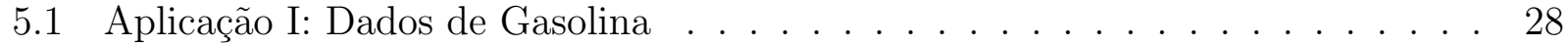

$5.2 \quad$ Aplicação II: Dados de Oxidação de Amônia . . . . . . . . . . . . . . . . . . . 37

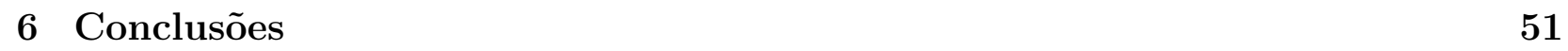

A Função Escore e Matriz de Informação de Fisher na Regressão Beta 53

\begin{tabular}{|lc}
\hline B Propriedades da Distribuição Simplex & 58
\end{tabular}

\begin{tabular}{|l|l|}
\hline C Conjunto de Dados & 61
\end{tabular}

\begin{tabular}{|lr}
\hline D Programas & 64 \\
\hline
\end{tabular}

D.1 Programas para ajuste do Modelo de Regressão Beta . . . . . . . . . . . . . . 64

D.2 Programas para ajuste do Modelo de Regressão Simplex ～. . . . . . . . . . . 68 
viii

\begin{tabular}{|l|l|}
\hline Referências Bibliográficas & 75
\end{tabular} 


\section{Capítulo 1}

\section{Introdução}

Diversas situações compreendem a análise de variáveis como proporções, percentagens ou frações. Podemos citar, por exemplo a proporção de casas que têm TV a cabo, porcentagem da renda gasta com alimentação, etc. Muitos modelos são estudados para analisar variáveis contínuas definidas no intervalo $(0,1)$, em função de outras variáveis, desde o modelo de regressão linear a modelos com distribuições mistas.

Kieschnick \& McCullough (2003) separam esse tipo de variável em duas categorias. Os dados da primeira categoria podem ser modelados usando uma distribuição contínua, pois estão definidos no intervalo aberto $(0,1)$, enquanto que na segunda, são definidos no intervalo fechado $[0,1]$ e seguem uma distribuição mista discreta-contínua. Aqui, vamos estudar variáveis que pertencem à primeira categoria. Nesta categoria, os autores apresentam sete modelos que identificaram como os mais utilizados para modelar essas variáveis. Esses sete modelos são os seguintes: o modelo normal linear, o modelo logito, o modelo normal censurado, o normal não linear, os modelos de regressão baseados na distribuição beta, distribuição simplex (Barndorff-Nielsen \& Jørgensen (1991)) e o de quasi-verossimilhança (ver Cox (1996) e Papke \& Wooldridge (1996)). Uma comparação desses modelos feita por Kieschnick \& McCullough (2003) indica que os modelos de regressão baseados na distribuição beta, na distribuição simplex ou o de quasi-verossimilhança são os que melhor se adequam aos dados de proporções e apresentam resultados inferenciais semelhantes. Dentre esses três modelos, uma variação do critério de seleção $A I C$ evidencia a superioridade do modelo de regressão beta. 
Neste trabalho, vamos apresentar o modelo de regressão beta definido por Ferrari \& Cribari-Neto (2004) e o modelo de regressão simplex. Vamos aplicar estes modelos a conjuntos de dados reais, avaliando-os por meio de algumas medidas de diagnóstico.

\section{$1.1 \quad$ Organização da Dissertação}

No Capítulo 2, apresentamos a definição da distribuição beta e o modelo de regressão beta definido por Ferrari \& Cribari-Neto (2004). Apresentamos, também, os resíduos propostos por Espinheira et al. (2008).

No Capítulo 3, apresentamos a distribuição simplex e desenvolvemos o modelo de regressão simplex utilizando a mesma metodologia do Capítulo 2. Baseados nos resíduos propostos por Espinheira et al. (2008), construímos um resíduo para esse modelo.

No Capítulo 4, propomos uma forma geral para algumas medidas de diagnóstico para a modelagem através da regressão beta ou da regressão simplex.

No Capítulo 5, aplicamos os modelos discutidos nos Capítulos 2 e 3 a dois conjuntos de dados reais. Avaliamos os modelos de regressão beta e simplex, por meio das medidas de diagnóstico apresentadas no Capítulo 4. A primeira aplicação refere-se a um estudo do processo de transformação do petróleo em gasolina. A segunda é sobre um estudo químico de oxidação de amônia.

No Capítulo 6, apresentamos as conclusões deste trabalho.

O Apêndice A apresenta alguns detalhes da obtenção da função escore e da matriz de informação de Fisher para o modelo de regressão beta. No Apêndice B, encontram-se algumas propriedades da distribuição simplex e, no Apêndice C, estão os conjuntos de dados utilizados nas aplicações do Capítulo 5. O Apêndice D apresenta algumas rotinas computacionais desenvolvidas para este trabalho. 


\subsection{Suporte Computacional}

Os programas utilizados para as aplicações do Capítulo 5 foram desenvolvidos no software R na versão 2.6.1. Esse pacote computacional encontra-se disponível gratuitamente em http://www.r-project.org.br e é muito utilizado, pois apresenta diversas funções implementadas, que também podem ser obtidas nesse endereço. Mais detalhes sobre R podem ser encontrados em R Development Core Team (2007).

Neste trabalho, os programas foram desenvolvidos com base em algumas funções dos pacotes betareg (Simas $(2006))$ e rmutil (Lindsey $(2007))$. 


\section{Capítulo 2}

\section{Modelo de Regressão Beta}

\subsection{Introdução}

Ferrari \& Cribari-Neto (2004) propuseram o modelo de regressão beta para situações em que a variável resposta é contínua e restrita ao intervalo $(0,1)$ e está relacionada a outras variáveis através de uma estrutura de regressão. O modelo proposto é baseado na suposição de que a resposta tem distribuição beta utilizando uma parametrização da lei beta que é indexada pela média e um parâmetro de dispersão.

\subsection{Distribuição Beta}

A distribuição beta é muito flexível em situações em que variável dependente é contínua e restrita ao intervalo $(0,1)$, pois sua função densidade pode assumir diferentes formas dependendo dos valores dos dois parâmetros que a compõem. A função densidade da distribuição beta, indexada pelos parâmetros $p$ e $q$, é definida como

$$
f(y ; p, q)=\frac{\Gamma(p+q)}{\Gamma(p) \Gamma(q)} y^{p-1}(1-y)^{q-1}, \quad 0<y<1, \quad p>0, \quad q>0,
$$


onde $\Gamma(p)$ é a função gama avaliada no ponto $p$

$$
\Gamma(p)=\int_{0}^{\infty} y^{p-1} e^{-y} d y
$$

A média e a variância de y são, respectivamente, dados por

$$
\mathrm{E}(y)=\frac{p}{p+q} \text { e } \operatorname{Var}(y)=\frac{p q}{(p+q)^{2}(p+q+1)}
$$

A distribuição uniforme é um caso particular de (2.1) quando $p=q=1$. A moda da distribuição beta é dada por

$$
y_{m}=\frac{p-1}{p+q-2}
$$

quando $p>1$ e $q>1$.

\subsection{Definição do Modelo de Regressão Beta}

O modelo de regressão beta proposto por Ferrari \& Cribari-Neto (2004) sugere uma reparametrização da distribuição beta, considerando-se a média da resposta e um parâmetro de dispersão. Sejam $\mu=p /(p+q)$ e $\phi=p+q$, isto é, $p=\mu \phi$ e $q=(1-\mu) \phi$. Assim, a densidade de $y$, em 2.1), pode ser escrita como

$$
f(y ; \mu, \phi)=\frac{\Gamma(\phi)}{\Gamma(\mu \phi) \Gamma((1-\mu) \phi)} y^{\mu \phi-1}(1-y)^{(1-\mu) \phi-1}, \quad 0<y<1
$$

em que $0<\mu<1$ e $\phi>0$. Assim, de (2.2), a média e a variância são dadas por

$$
\mathrm{E}(y)=\mu \quad \mathrm{e} \operatorname{Var}(y)=\frac{V(\mu)}{1+\phi}
$$

em que $V(\mu)=\mu(1-\mu)$ é a função de variância, $\mu$ é a média da variável resposta e $\phi$ pode ser interpretado como um parâmetro de precisão (ou dispersão) no sentido que, para $\mu$ fixado, quanto maior for o valor de $\phi$, menor a variância de $y$.

Com isso, a densidade beta pode ter diferentes formas de acordo com os valores dos parâmetros $(\mu, \phi)$, apresentando simetria (quando $\mu=1 / 2$ ) ou assimetria (quando $\mu \neq 1 / 2$ ). 

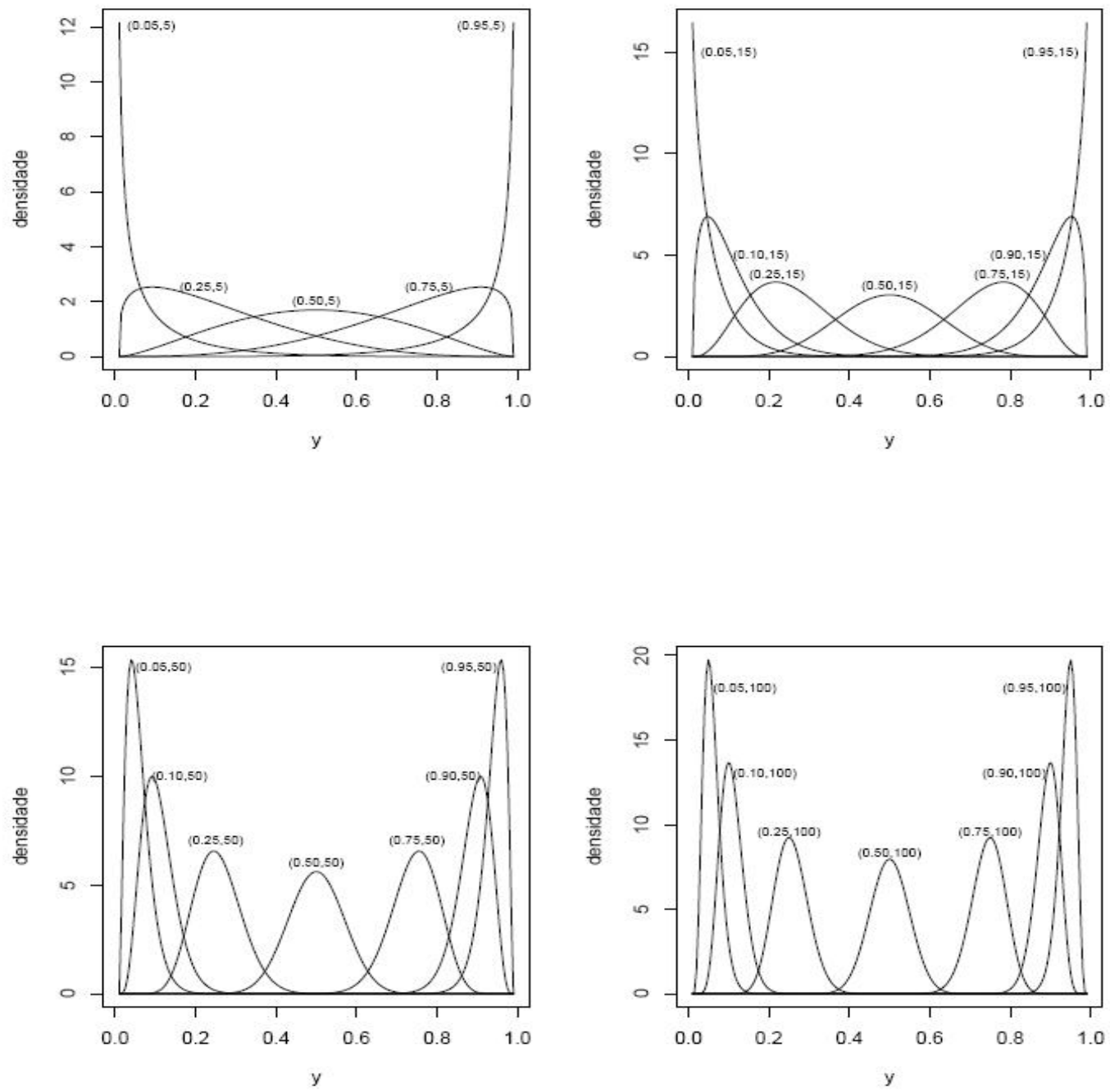

Figura 2.1: Densidades beta para diferentes combinações de $(\mu, \phi)$.

A Figura 2.1. extraída de Ferrari \& Cribari-Neto (2004), mostra, por exemplo, alguns formatos como $J, J$ invertido e $U$. Além disso, para $\mu$ fixado, a dispersão da distribuição decresce quando $\phi$ cresce. A distribuição uniforme ocorre quando $\mu=0,5$ e $\phi=2$.

O modelo proposto também pode ser utilizado quando a variável resposta não está restrita ao intervalo unitário padrão $(0,1)$, em situações em que a resposta é restrita ao intervalo $(a, b)$, onde $a$ e $b$ são constantes conhecidas, $a<b$. Neste caso, modelamos $(y-a) /(b-a)$ no lugar de modelar $y$ diretamente. 
Sejam $y_{1}, \ldots, y_{n}$ variáveis aleatórias independentes, onde cada $y_{t}, t=1, \ldots, n$, segue uma distribuição beta com média $\mu_{t}$ e parâmetro de precisão desconhecido $\phi$ (constante para todo t), cuja densidade é dada por (2.3). O modelo de regressão beta é definido por (2.3) e pelo componente sistemático

$$
g\left(\mu_{t}\right)=\sum_{i=1}^{k} x_{t i} \beta_{i}=\eta_{t}
$$

em que $\eta_{t}=x_{t}^{\top} \beta$ é o preditor linear, $\beta=\left(\beta_{1}, \ldots, \beta_{k}\right)^{\top}$ é um vetor de parâmetros desconhecidos a serem estimados $\left(\beta \in \Re^{k}\right), x_{t}^{\top}=\left(x_{t 1}, \ldots, x_{t k}\right)$ representa os valores de $k(k<n)$ variáveis explicativas que são assumidos fixos e conhecidos. A funçao $g(\cdot)$ é chamada de função de ligação, uma função estritamente monótona e duplamente diferenciável que transforma valores do intervalo $(0,1)$ em $\Re$.

Podemos notar que a variância de $y_{t}$ é uma função de $\mu_{t}$ e, como conseqüência, das variáveis explicativas $x_{t}$. Portanto, variáveis respostas com variâncias não constantes são naturalmente acomodadas no modelo.

Uma função de ligação muito utilizada é a logito, pois permite interpretação simples para os parâmetros de regressão. Em (2.4), segue que

$$
g\left(\mu_{t}\right)=\log \left(\frac{\mu_{t}}{1-\mu_{t}}\right)=x_{t}^{\top} \beta, t=1, \ldots, n .
$$

Então,

$$
\frac{\mu_{t}}{1-\mu_{t}}=\exp \left(x_{t}^{\top} \beta\right)
$$

e podemos escrever

$$
\mu_{t}=\frac{\exp \left(x_{t}^{\top} \beta\right)}{1+\exp \left(x_{t}^{\top} \beta\right)}
$$

A expressão (2.5) é a função inversa de $g\left(\mu_{t}\right)$. Quando a função logito é usada, os parâmetros de regressão podem ser interpretados em termos da razão de chances (odds ratio). Suponha que o valor da $i$-ésima variável regressora é aumentado por $c$ unidades, enquanto que as demais variáveis independentes permanecem inalteradas. Denotemos $\mu^{\dagger}$ como a média de $y$ sob este novo valor das covariadas, sendo $\mu$ a média de $y$ sob o valor original das covariadas, dessa forma temos

$$
\frac{\mu^{\dagger}}{1-\mu^{\dagger}}=\exp \left(x_{t 1} \beta_{1}+\ldots+\left(x_{t i}+c\right) \beta_{i}+\ldots+x_{t k} \beta_{k}\right) .
$$


A razão de chances é dada por

$$
\exp \left(c \beta_{i}\right)=\frac{\mu^{\dagger} /\left(1-\mu^{\dagger}\right)}{\mu /(1-\mu)}
$$

Um estudo sobre a escolha da função de ligação e os efeitos de sua especificação incorreta foi feita por Andrade (2007). A utilização da função logito mesmo quando está especificada incorretamente resulta em estimativas para as respostas médias com vieses pequenos. Mas, se não há interesse na interpretação dos parâmetros, a escolha da função de ligação de ArandaOrdaz pode ser mais adequada.

\subsubsection{Função Escore e Matriz de Informação de Fisher}

Nesta seção, apresentamos a função escore e a matriz de informação de Fisher para o vetor de parâmetros $\beta$ e, também, para o parâmetro de precisão $\phi$, que são obtidas através das derivadas do logaritmo natural da função de verossimilhança com respeito a $\beta$ e $\phi$.

O logaritmo natural da função de verossimilhança baseada em uma amostra de $n$ observações independentes é

$$
\ell(\beta, \phi)=\sum_{t=1}^{n} \ell_{t}\left(\mu_{t}, \phi\right)
$$

em que

$$
\begin{aligned}
\ell_{t}\left(\mu_{t}, \phi\right) & =\log \Gamma(\phi)-\log \Gamma\left(\mu_{t} \phi\right)-\log \Gamma\left(\left(1-\mu_{t}\right) \phi\right)+\left(\mu_{t} \phi-1\right) \log y_{t} \\
& +\left\{\left(1-\mu_{t}\right) \phi-1\right\} \log \left(1-y_{t}\right)
\end{aligned}
$$

com $\mu_{t}=g^{-1}\left(\eta_{t}\right)$ definido em 2.4.

Sejam $y_{t}^{*}$ e $\mu_{t}^{*}$ dados por

$$
y_{t}^{*}=\log \left\{\frac{y_{t}}{\left(1-y_{t}\right)}\right\} \quad \text { e } \quad \mu_{t}^{*}=\psi\left(\mu_{t} \phi\right)-\psi\left(\left(1-\mu_{t}\right) \phi\right)
$$

sendo $\psi($.$) a função digama definida como$

$$
\psi(a)=\frac{\mathrm{d} \log \Gamma(a)}{\mathrm{d} a}=\frac{\Gamma^{\prime}(a)}{\Gamma(a)},
$$


onde $\Gamma^{\prime}(a)=\mathrm{d} \Gamma(a) / \mathrm{d} a, a>0$.

A partir da primeira derivada do logaritmo da função de verossimilhança em relação a $\beta_{i}, i=1, \ldots, k$, obtemos a função escore para $\beta$, que matricialmente é dada por

$$
U_{\beta}(\beta, \phi)=\phi X^{\top} T\left(y^{*}-\mu^{*}\right)
$$

em que $X$ é uma matriz $n \times k$, cuja $t$-ésima linha é $x_{t}^{\top}, T=\operatorname{diag}\left\{1 / g^{\prime}\left(\mu_{1}\right), \ldots, 1 / g^{\prime}\left(\mu_{n}\right)\right\}$, com $g^{\prime}\left(\mu_{t}\right)=\mathrm{d} \eta_{t} / \mathrm{d} \mu_{t}, y^{*}=\left(y_{1}^{*}, \ldots, y_{n}^{*}\right)^{\top}$ e $\mu^{*}=\left(\mu_{1}^{*}, \ldots, \mu_{n}^{*}\right)^{\top}$.

Analogamente, a função escore para o parâmetro de precisão $\phi$ pode ser obtida da primeira derivada de $\ell_{t}(\beta, \phi)$ em relação a $\phi$

$$
U_{\phi}(\beta, \phi)=\sum_{t=1}^{n}\left\{\mu_{t}\left(y_{t}^{*}-\mu_{t}^{*}\right)+\log \left(1-y_{t}\right)-\psi\left(\left(1-\mu_{t}\right) \phi\right)+\psi(\phi)\right\}
$$

sendo $U_{\phi}(\beta, \phi)$ um escalar.

A matriz de informação de Fisher para $(\beta, \phi)$, obtida através das derivadas de segunda ordem de $\ell(\beta, \phi)$ em relação a $\beta_{i}$ e $\beta_{j}$, a $\beta_{i}$ e $\phi$, e com respeito a $\phi$, é dada por

$$
K=K(\beta, \phi)=\left(\begin{array}{cc}
K_{\beta \beta} & K_{\beta \phi} \\
K_{\phi \beta} & K_{\phi \phi}
\end{array}\right)
$$

onde $K_{\beta \beta}=\phi X^{\top} W X, K_{\beta \phi}=K_{\phi \beta}=X^{\top} T c$ e $K_{\phi \phi}=\operatorname{tr}(D)$, sendo

$$
\begin{aligned}
W & =\operatorname{diag}\left\{w_{1}, \ldots, w_{n}\right\}, \operatorname{com} w_{t}=\phi\left\{\psi^{\prime}\left(\mu_{t} \phi\right)+\psi^{\prime}\left(\left(1-\mu_{t}\right) \phi\right)\right\} \frac{1}{\left\{g^{\prime}\left(\mu_{t}\right)\right\}^{2}}, \\
c & =\left(c_{1}, \ldots, c_{n}\right)^{\top}, \operatorname{com} c_{t}=\phi\left\{\psi^{\prime}\left(\mu_{t} \phi\right) \mu_{t}-\psi^{\prime}\left(\left(1-\mu_{t}\right) \phi\right)\left(1-\mu_{t}\right)\right\}, \\
D & =\operatorname{diag}\left\{d_{1}, \ldots, d_{n}\right\}, \operatorname{com} d_{t}=\psi^{\prime}\left(\mu_{t} \phi\right) \mu_{t}^{2}+\psi^{\prime}\left(\left(1-\mu_{t}\right) \phi\right)\left(1-\mu_{t}\right)^{2}-\psi^{\prime}(\phi),
\end{aligned}
$$

em que $\psi^{\prime}(\cdot)$ é a função trigama definida como $\psi^{\prime}(a)=\mathrm{d} \psi(a) / \mathrm{d} a, a>0$.

Sob condições gerais de regularidade (ver Sen \& Singer (1993)), quando o tamanho da amostra é grande, a distribuição aproximada dos estimadores de máxima verossimilhança é 
dada por

$$
\left(\begin{array}{l}
\widehat{\beta} \\
\widehat{\phi}
\end{array}\right) \sim N_{k+1}\left(\left(\begin{array}{l}
\beta \\
\phi
\end{array}\right), K^{-1}\right)
$$

onde $\widehat{\beta}$ e $\widehat{\phi}$ são, respectivamente, os estimadores de máxima verossimilhança de $\beta$ e $\phi$. Para obtermos os erros padrões assintóticos para as estimativas de máxima verossimilhança, é útil escrevermos $K^{-1}$ de forma explícita. Utilizando as expressões para as inversas de matrizes subdivididas dadas por Rao (1973), obtém-se

$$
K^{-1}=K^{-1}(\beta, \phi)=\left(\begin{array}{cc}
K^{\beta \beta} & K^{\beta \phi} \\
K^{\phi \beta} & K^{\phi \phi}
\end{array}\right),
$$

onde

$$
K^{\beta \beta}=\frac{1}{\phi}\left(X^{\top} W X\right)^{-1}\left\{I_{k}+\frac{X^{\top} T c c^{\top} T^{\top} X\left(X^{\top} W X\right)^{-1}}{\gamma \phi}\right\},
$$

com $\gamma=\operatorname{tr}(D)-\phi^{-1} c^{\top} T^{\top} X\left(X^{\top} W X\right)^{-1} X^{\top} T c$, e $I_{k}$ é a matriz identidade de ordem $k$,

$$
K^{\beta \phi}=\left(K^{\phi \beta}\right)^{\top}=-\frac{1}{\gamma \phi}\left(X^{\top} W X\right)^{-1} X^{\top} T c \text { e } K^{\phi \phi}=\gamma^{-1}
$$

\subsubsection{Estimação dos parâmetros}

Os estimadores de máxima verissimilhança de $\beta$ e $\phi$ são obtidos das equações $U_{\beta}(\beta, \phi)=0$ e $U_{\phi}(\beta, \phi)=0$, contudo não apresentam uma solução analítica com forma fechada. Logo, os estimadores precisam ser obtidos através de maximização numérica do logaritmo da função de verossimilhança, utilizando algum algoritmo de otimização, como o de Newton-Raphson, quasi-Newton (método BFGS) ou escore de Fisher. Nesta seção, descreveremos sucintamente os métodos de Newton-Raphson e BFGS, este utilizado na library betareg do software $R$ usado nas aplicações.

Denotemos $\theta=\left(\beta^{\top}, \phi\right)^{\top}$ como o vetor de parâmetros e $U(\theta)=\left(U_{\beta}(\beta, \phi)^{\top}, U_{\phi}(\beta, \phi)\right)^{\top}$, o vetor das funções escore de dimensão $(k+1) \times 1$. O processo iterativo de Newton-Raphson é dado por

$$
\theta^{(m+1)}=\theta^{(m)}+\left\{-U^{\prime}\left(\theta^{(m)}\right)\right\}^{-1} U\left(\theta^{(m)}\right), \quad m=0,1, \ldots
$$

O método de quasi-Newton é mais conhecido como método BFGS, pois foi desenvolvido 
por Broyden, Fletcher, Goldfarb e Shanno (ver Nocedal \& Wright (1999)). Este método, com o mesmo princípio do algoritmo de Newton-Raphson, utiliza uma seqüência de matrizes simétricas e positivas definidas $B^{(m)}$ no lugar da matriz $U^{\prime}\left(\theta^{(m)}\right)^{-1}$. A forma iterativa é dada por

$$
\theta^{(m+1)}=\theta^{(m)}-\alpha^{(m)} B^{(m)} U\left(\theta^{(m)}\right), \quad m=0,1, \ldots
$$

onde $\alpha^{(m)}$ é um escalar determinado por algum procedimento de busca linear a partir de $\theta^{(m)}$ na direção $-B^{(m)} U\left(\theta^{(m)}\right)$ de forma que $f\left(y ; \theta^{(m)}\right)$ cresça nessa direção. Outros detalhes sobre $B^{(m)}$ e sobre o método também podem ser encontrados em Oliveira (2004).

Os procedimentos iterativos são sensíveis à estimativa inicial $\theta^{(0)}$. Nas aplicações da regressão beta, foi utilizado a estimativa inicial sugerida por Ferrari \& Cribari-Neto (2004).

\subsection{Intervalos de Confiança}

Nesta seção apresentamos alguns intervalos de confiança para os parâmetros de interesse, considerando a distribuição assitóntica dos estimadores de máxima verossimilhança $\widehat{\beta}$ e $\widehat{\phi}$, dada por 2.11). Um intervalo de confiança de aproximadamente $(1-\alpha) \times 100 \%$ para $\beta_{i}, i=$ $1, \ldots, k$ e $0<\alpha<1$, tem limites dado por

$$
\widehat{\beta}_{i} \pm \Phi^{-1}(1-\alpha / 2) \operatorname{se}\left(\widehat{\beta}_{i}\right)
$$

em que $s e\left(\widehat{\beta}_{i}\right)$ é o erro padrão para $\widehat{\beta}_{i}$ obtido pela raiz quadrada do $i$-ésimo elemento da diagonal da matriz $\hat{K}^{-1}$. Similarmente para $\phi$, um intervalo de confiança aproximado de $(1-\alpha) \times 100 \%$ pode ser obtido por

$$
\widehat{\phi} \pm \Phi^{-1}(1-\alpha / 2) \operatorname{se}(\widehat{\phi})
$$

em que $\operatorname{se}(\widehat{\phi})=\widehat{\gamma}^{-1 / 2}$ é o erro padrão para $\widehat{\phi}$ obtido pelo $(k+2)$-ésimo elemento da diagonal da matriz $\hat{K}^{-1}$. Finalmente, um intervalo de confiança de aproximadamente $(1-\alpha) \times 100 \%$ para $\mu_{t}$ tem limites

$$
\left[g^{-1}\left(\widehat{\eta}_{t}-\Phi^{-1}(1-\alpha / 2) s e\left(\widehat{\eta}_{t}\right)\right), g^{-1}\left(\widehat{\eta}_{t}+\Phi^{-1}(1-\alpha / 2) s e\left(\widehat{\eta}_{t}\right)\right)\right]
$$


em que $\widehat{\eta}_{t}=x_{t}^{\top} \widehat{\beta}$ e o erro padrão de $\widehat{\eta}_{t}$ é dado por $\operatorname{se}\left(\widehat{\eta}_{t}\right)=\sqrt{x_{t}^{\top} \widehat{\operatorname{cov}}(\widehat{\beta}) x_{t}}$, sendo $\operatorname{cov}(\widehat{\beta})=K^{\beta \beta}$, uma submatriz de $K^{-1}$ definida em (2.12).

\subsection{Resíduos}

Os resíduos são a base da análise de diagnóstico descrita no Capítulo 4, uma etapa importante na análise de regressão para a validação do modelo.

Nesta seção, apresentamos os resíduos propostos por Espinheira et al. (2008) e Ferrari \& Cribari-Neto (2004). Para a definição desses resíduos foi considerado o modelo definido em (2.3) com $\phi$ conhecido.

\subsubsection{Resíduo ordinário}

O resíduo ordinário padronizado foi definido por Ferrari \& Cribari-Neto (2004) como

$$
r_{t}=\frac{y_{t}-\widehat{\mu}_{t}}{\sqrt{\widehat{\operatorname{Var}}\left(y_{t}\right)}}
$$

em que $\widehat{\operatorname{Var}}\left(y_{t}\right)=\widehat{\mu}_{t}\left(1-\widehat{\mu}_{t}\right) /(1+\widehat{\phi}), \widehat{\mu}_{t}=g^{-1}\left(x_{t}^{\top} \widehat{\beta}\right)$, e $\widehat{\beta}$ e $\widehat{\phi}$ são os estimadores de máxima verossimilhança de $\beta$ e $\phi$, respectivamente.

\subsubsection{Resíduo ponderado padronizado 1}

Este resíduo foi baseado no método iterativo scoring de Fisher para estimação de $\beta$, com $\phi$ conhecido. O processo iterativo é dado por

$$
\beta^{(m+1)}=\beta^{(m)}+\left(X^{\top} W^{(m)} X\right)^{-1} X^{\top} T^{(m)}\left(y^{*}-\mu^{*(m)}\right),
$$

com $y^{*}, \mu^{*}, T$ e $W$ definidos em (2.7), 2.8) e 2.10).

Reescrevendo o processo iterativo 2.13 em termos de regressão de mínimos quadrados ponderados: $\beta^{(m+1)}=\left(X^{\top} W^{(m)} X\right)^{-1} X^{\top} W^{(m)} z^{(m)}$, onde $z^{(m)}=\eta^{(m)}+W^{-1(m)} T^{(m)}\left(y^{*}-\mu^{*(m)}\right)$, 
com $\eta=\left(\eta_{1}, \ldots, \eta_{n}\right)^{\top}=X \beta$. Na convergência, segue que

$$
\widehat{\beta}=\left(X^{\top} \widehat{W} X\right)^{-1} X^{\top} \widehat{W} z
$$

com

$$
z=\widehat{\eta}+\widehat{W}^{-1} \widehat{T}\left(y^{*}-\widehat{\mu}^{*}\right)
$$

onde $\widehat{W}$ e $\widehat{T}$ são as matrizes $W$ e $T$ avaliadas no estimador de máxima verossimilhança.

Assim, usando as definições de $T$ e $W$ em (2.8) e (A.8), Espinheira et al. (2008) definem o resíduo ponderado como

$$
r_{t}^{*}=\frac{y_{t}^{*}-\widehat{\mu}_{t}^{*}}{\sqrt{\phi v_{t}}}
$$

$\operatorname{com} v_{t}=\psi^{\prime}\left(\widehat{\mu}_{t} \phi\right)+\psi^{\prime}\left(\left(1-\widehat{\mu}_{t}\right) \phi\right)$

Como $\operatorname{Var}\left(y_{t}^{*}\right)=\psi^{\prime}\left(\mu_{t} \phi\right)+\psi^{\prime}\left(\left(1-\mu_{t}\right) \phi\right)$, o resíduo ponderado padronizado 1 definido por Ferrari \& Cribari-Neto (2004) é dado por

$$
r_{t}^{p}=\phi^{1 / 2} r_{t}^{*}=\frac{y_{t}^{*}-\widehat{\mu}_{t}^{*}}{\sqrt{v_{t}}} .
$$

\subsubsection{Resíduo ponderado padronizado 2}

Outro resíduo definido por Espinheira et al. (2008) se baseia na variância de z. Para isso, reescrevemos 2.14) como $\left(X^{\top} \widehat{W} X\right) \widehat{\beta}=X^{\top} \widehat{W} z$. Como $\operatorname{cov}(\widehat{\beta})=\phi^{-1}\left(X^{\top} W X\right)^{-1}$, segue que $\operatorname{cov}(z) \approx \phi^{-1} \widehat{W^{-1}}$. Assim, obtemos $\operatorname{cov}\left(r^{*}\right) \approx \phi^{-1}\left(I_{n}-H\right)$, onde $I_{n}$ é a matriz identidade de ordem $n$ e $H=\widehat{W}^{1 / 2} X\left(X^{\top} \widehat{W} X\right)^{-1} X^{\top} \widehat{W}^{1 / 2}$. O resíduo ponderado padronizado 2 é dado por

$$
r_{t}^{p p}=\frac{r_{t}^{*}}{\sqrt{\phi^{-1}\left(1-h_{t t}\right)}}=\frac{r_{t}^{p}}{\sqrt{\left(1-h_{t t}\right)}}=\frac{y_{t}^{*}-\widehat{\mu}_{t}^{*}}{\sqrt{v_{t}\left(1-h_{t t}\right)}}
$$

em que $h_{t t}$ é o $t$-ésimo elemento da diagonal de $H$.

Note que foi considerado $\phi$ fixo. Na prática, para calcularmos esses resíduos, devemos substituir $\phi$ pela sua estimativa de máxima verossimilhança $\widehat{\phi}$.

O resíduo mais eficiente na identificação de observações que têm maior influência nas estimativas dos parâmetros é o resíduo ponderado padronizado 2. Através de um estudo com 
dados simulados, em que foi incluído um valor atípico, Espinheira et al. (2008) mostram que o resíduo ponderado padronizado 2 é capaz de identificar melhor uma observação claramente atípica, sendo, ao mesmo tempo, um ponto leverage e outlier. 


\section{Capítulo 3}

\section{Modelo de Regressão Simplex}

\subsection{Introdução}

Outra distribuição que, também, pode ser utilizada para estudar uma variável resposta contínua e restrita ao intervalo $(0,1)$ é a distribuição simplex (Barndorff-Nielsen \& Jørgensen (1991)). A distribuição simplex faz parte dos modelos de dispersão (Jørgensen (1997)), que estendem os modelos lineares generalizados.

Neste capítulo será apresentado o modelo de regressão baseado na distribuição simplex, as funções escore e a matriz de informação de Fisher para os parâmetros da regressão, os estimadores dos parâmetros e algumas propriedades.

\subsection{Distribuição Simplex}

Uma variável aleatória $y$ que segue uma distribuição simplex com média $\mu \in(0,1)$ e parâmetro de dispersão $\sigma^{2}>0$ tem função densidade dada por

$$
p\left(y ; \mu, \sigma^{2}\right)=\left[2 \pi \sigma^{2}\{y(1-y)\}^{3}\right]^{-1 / 2} \exp \left\{-\frac{1}{2 \sigma^{2}} d(y ; \mu)\right\}, y \in(0,1),
$$


em que

$$
d(y ; \mu)=\frac{(y-\mu)^{2}}{y(1-y) \mu^{2}(1-\mu)^{2}} .
$$

Denotamos a distribuição de $y$ por $S^{-}\left(\mu, \sigma^{2}\right)$. A variância de $y$ é dada por

$$
\operatorname{var}(y)=\mu(1-\mu)-\frac{1}{\sqrt{2} \sigma} \exp \left\{\frac{1}{\sigma^{2} \mu^{2}(1-\mu)^{2}}\right\} \Gamma\left\{\frac{1}{2}, \frac{1}{2 \sigma^{2} \mu^{2}(1-\mu)^{2}}\right\}
$$

em que $\Gamma(a, b)$ é a função gama incompleta definida por $\Gamma(a, b)=\int_{b}^{\infty} t^{a-1} e^{-t} d t$. Definimos a função de variância de $y$ como $V(\mu)=\mu^{3}(1-\mu)^{3}$.

\subsection{Definição do Modelo de Regressão Simplex}

Sejam $y_{1}, \ldots, y_{n}$ variáveis aleatórias independentes, sendo cada $y_{t} \sim S^{-}\left(\mu_{t}, \sigma^{2}\right), t=$ $1, \ldots, n$. O modelo de regressão simplex é definido pela densidade da forma (3.1), sendo as médias $\mu_{t}$ modeladas por

$$
g\left(\mu_{t}\right)=\sum_{i=1}^{k} x_{t i} \beta_{i}=x_{t}^{\top} \beta=\eta_{t}
$$

em que $g($.$) é a função de ligação, estritamente monótona e duplamente diferenciável que$ transforma valores do intervalo $(0,1)$ em $\Re, \beta=\left(\beta_{1}, \ldots, \beta_{k}\right)^{\top}$ é o vetor dos parâmetros da regressão $\left(\beta \in \Re^{k}\right), x_{t}^{\top}=\left(x_{t 1}, \ldots, x_{t k}\right)$ são os valores conhecidos de $k$ covariáveis e $\eta_{t}$ é o preditor linear.

Podemos observar que o modelo de regressão simplex também acomoda variáveis respostas com variâncias não constantes, assim como o modelo de regressão beta, pois a variância de $y_{t}$ é uma função de $\mu_{t}$ (definida em (3.2)).

\subsubsection{Função Escore e Matriz de Informação de Fisher}

Nesta seção, apresentamos a função escore e a matriz de informação de Fisher para o modelo de regressão simplex. Estas quantidades são obtidas da mesma forma que no modelo de regressão beta, ou seja, a partir da função de verossimilhança. 
Baseado em uma amostra de $n$ observações independentes, o logaritmo da função de verossimilhança é dado por

$$
\ell\left(\beta, \sigma^{2}\right)=\sum_{t=1}^{n} \ell_{t}\left(\mu_{t}, \sigma^{2}\right)
$$

em que

$$
\begin{aligned}
\ell_{t}\left(\mu_{t}, \sigma^{2}\right) & =-\frac{1}{2} \log \left[2 \pi \sigma^{2}\left\{y_{t}\left(1-y_{t}\right)\right\}^{3}\right]-\frac{1}{2 \sigma^{2}} d\left(y_{t} ; \mu_{t}\right) \\
& =-\frac{1}{2} \log (2 \pi)-\frac{1}{2} \log \sigma^{2}-\frac{3}{2} \log \left\{y_{t}\left(1-y_{t}\right)\right\}-\frac{1}{2 \sigma^{2}} d\left(y_{t} ; \mu_{t}\right) .
\end{aligned}
$$

Derivando $\ell\left(\beta, \sigma^{2}\right)$ em relação a $\beta_{i}, i=1, \ldots, k$, temos

$$
\frac{\partial \ell\left(\beta, \sigma^{2}\right)}{\partial \beta_{i}}=\sum_{t=1}^{n} \frac{\partial \ell_{t}\left(\mu_{t}, \sigma^{2}\right)}{\partial \mu_{t}} \frac{\mathrm{d} \mu_{t}}{\mathrm{~d} \eta_{t}} \frac{\partial \eta_{t}}{\partial \beta_{i}}
$$

onde

$$
\frac{\partial \ell_{t}\left(\mu_{t}, \sigma^{2}\right)}{\partial \mu_{t}}=-\frac{1}{2 \sigma^{2}} d^{\prime}\left(y_{t} ; \mu_{t}\right), \quad \text { sendo } \quad d^{\prime}\left(y_{t} ; \mu_{t}\right)=\frac{\partial d\left(y_{t} ; \mu_{t}\right)}{\partial \mu_{t}} .
$$

Definindo

$$
u_{t}=-\frac{1}{2} d^{\prime}\left(y_{t} ; \mu_{t}\right), t=1, \ldots, n,
$$

temos que

$$
\frac{\partial \ell_{t}\left(\mu_{t}, \sigma^{2}\right)}{\partial \mu_{t}}=\sigma^{-2} u_{t}
$$

Temos em (3.4) que

$$
\frac{\partial \ell\left(\beta, \sigma^{2}\right)}{\partial \beta_{i}}=\sum_{t=1}^{n} \sigma^{-2} u_{t} \frac{1}{g^{\prime}\left(\mu_{t}\right)} x_{t i} \text {, em que } \frac{\mathrm{d} \mu_{t}}{\mathrm{~d} \eta_{t}}=\frac{1}{g^{\prime}\left(\mu_{t}\right)} \text { e } \frac{\partial \eta_{t}}{\partial \beta_{i}}=x_{t i} \text {. }
$$

Em notação matricial, a função escore para $\beta$ é dada por

$$
U_{\beta}\left(\beta, \sigma^{2}\right)=\sigma^{-2} X^{\top} T u
$$

onde $X$ é um matriz $n \times k$, cuja $t$-ésima linha é $x_{t}^{\top}, T=\operatorname{diag}\left\{1 / g^{\prime}\left(\mu_{1}\right), \ldots, 1 / g^{\prime}\left(\mu_{n}\right)\right\}$ e $u^{\top}=\left(u_{1}, \ldots, u_{n}\right)^{\top}$. 
Para obtermos a função escore para $\sigma^{2}$, derivamos $\ell\left(\beta, \sigma^{2}\right)$ em relação a $\sigma^{2}$,

$$
\frac{\partial \ell\left(\beta, \sigma^{2}\right)}{\partial \sigma^{2}}=\sum_{t=1}^{n} \frac{\partial \ell_{t}\left(\mu_{t}, \sigma^{2}\right)}{\partial \sigma^{2}}
$$

em que

$$
\frac{\partial \ell_{t}\left(\mu_{t}, \sigma^{2}\right)}{\partial \sigma^{2}}=-\frac{1}{2 \sigma^{2}}+\frac{1}{2 \sigma^{4}} d\left(y_{t} ; \mu_{t}\right)
$$

Logo, a função escore para $\sigma^{2}$ é dada por

$$
U_{\sigma^{2}}\left(\beta, \sigma^{2}\right)=\sum_{t=1}^{n}\left[-\frac{1}{2 \sigma^{2}}+\frac{1}{2 \sigma^{4}} d\left(y_{t} ; \mu_{t}\right)\right]=-\frac{n}{2 \sigma^{2}}+\frac{1}{2 \sigma^{4}} \sum_{t=1}^{n} d\left(y_{t} ; \mu_{t}\right) .
$$

A partir das segundas derivadas do logaritmo da função de verossimilhança definido em (3.3), obtemos a matriz de informação de Fisher. De (3.4), a segunda derivada de $l\left(\beta, \sigma^{2}\right)$ em relação a $\beta_{i}$ e $\beta_{j}$ é dada por

$$
\begin{aligned}
\frac{\partial^{2} \ell\left(\beta, \sigma^{2}\right)}{\partial \beta_{i} \partial \beta_{j}} & =\sum_{t=1}^{n} \frac{\partial}{\partial \mu_{t}}\left(\frac{\partial \ell_{t}\left(\mu_{t}, \sigma^{2}\right)}{\partial \mu_{t}} \frac{\mathrm{d} \mu_{t}}{\mathrm{~d} \eta_{t}}\right) \frac{\mathrm{d} \mu_{t}}{\mathrm{~d} \eta_{t}} \frac{\partial \eta_{t}}{\partial \beta_{j}} x_{t i} \\
& =\sum_{t=1}^{n}\left\{\frac{\partial^{2} \ell_{t}\left(\mu_{t}, \sigma^{2}\right)}{\partial \mu_{t}^{2}} \frac{\mathrm{d} \mu_{t}}{\mathrm{~d} \eta_{t}}+\frac{\partial \ell_{t}\left(\mu_{t}, \sigma^{2}\right)}{\partial \mu_{t}} \frac{\partial}{\partial \mu_{t}}\left(\frac{\mathrm{d} \mu_{t}}{\mathrm{~d} \eta_{t}}\right)\right\} \frac{\mathrm{d} \mu_{t}}{\mathrm{~d} \eta_{t}} x_{t i} x_{t j}
\end{aligned}
$$

Como $\mathrm{E}\left(\partial \ell_{t}\left(\mu_{t}, \sigma^{2}\right) / \partial \mu_{t}\right)=0$, temos que

$$
\mathrm{E}\left(\frac{\partial^{2} \ell\left(\beta, \sigma^{2}\right)}{\partial \beta_{i} \partial \beta_{j}}\right)=\sum_{t=1}^{n} \mathrm{E}\left(\frac{\partial^{2} \ell_{t}\left(\mu_{t}, \sigma^{2}\right)}{\partial \mu_{t}^{2}}\right)\left(\frac{\mathrm{d} \mu_{t}}{\mathrm{~d} \eta_{t}}\right)^{2} x_{t i} x_{t j}
$$

Da equação 3.5, obtemos

$$
\frac{\partial^{2} \ell_{t}\left(\mu_{t}, \sigma^{2}\right)}{\partial \mu_{t}^{2}}=-\frac{1}{2 \sigma^{2}} d^{\prime \prime}\left(y_{t} ; \mu_{t}\right), \quad \text { sendo } \quad d^{\prime \prime}\left(y_{t} ; \mu_{t}\right)=\frac{\partial^{2} d\left(y_{t} ; \mu_{t}\right)}{\partial \mu_{t}^{2}} .
$$

Pela Propriedade (B.4), temos que

$$
\frac{1}{2} \mathrm{E}\left[d^{\prime \prime}\left(y_{t} ; \mu_{t}\right)\right]=\frac{3 \sigma^{2}}{\mu_{t}\left(1-\mu_{t}\right)}+\frac{1}{\mu_{t}^{3}\left(1-\mu_{t}\right)^{3}} .
$$


Logo,

$$
\mathrm{E}\left(\frac{\partial^{2} \ell\left(\beta, \sigma^{2}\right)}{\partial \beta_{i} \partial \beta_{j}}\right)=-\frac{1}{\sigma^{2}} \sum_{t=1}^{n} a_{t} x_{t i} x_{t j}
$$

com

$$
a_{t}=\left\{\frac{3 \sigma^{2}}{\mu_{t}\left(1-\mu_{t}\right)}+\frac{1}{\mu_{t}^{3}\left(1-\mu_{t}\right)^{3}}\right\} \frac{1}{\left\{g^{\prime}\left(\mu_{t}\right)\right\}^{2}} .
$$

Matricialmente,

$$
\mathrm{E}\left(\frac{\partial^{2} \ell\left(\beta, \sigma^{2}\right)}{\partial \beta \partial \beta^{\top}}\right)=-\frac{1}{\sigma^{2}} X^{\top} A X, \operatorname{com} A=\operatorname{diag}\left\{a_{1}, \ldots, a_{n}\right\} .
$$

De (3.4), a derivada de segunda ordem de $\ell\left(\beta, \sigma^{2}\right)$ com respeito a $\beta$ e $\sigma^{2}$ é dada por

$$
\frac{\partial^{2} \ell\left(\beta, \sigma^{2}\right)}{\partial \beta_{i} \partial \sigma^{2}}=\sum_{t=1}^{n} \frac{\partial}{\partial \sigma^{2}}\left[\sigma^{-2} u_{t} \frac{1}{g^{\prime}\left(\mu_{t}\right)} x_{t i}\right]=-\frac{1}{\sigma^{4}} \sum_{t=1}^{n} u_{t} \frac{1}{g^{\prime}\left(\mu_{t}\right)} x_{t i} .
$$

Como $\mathrm{E}\left(u_{t}\right)=0($ Propriedade $(\mathrm{B} .5))$, segue-se

$$
\mathrm{E}\left(\frac{\partial^{2} \ell\left(\beta, \sigma^{2}\right)}{\partial \beta \partial \sigma^{2}}\right)=0
$$

Finalmente, derivando 3.8 em relação a $\sigma^{2}$, obtemos

$$
\begin{aligned}
\frac{\partial^{2} \ell\left(\beta, \sigma^{2}\right)}{\partial \sigma^{4}} & =\sum_{t=1}^{n} \frac{\partial \ell_{t}^{2}\left(\mu_{t}, \sigma^{2}\right)}{\partial \sigma^{4}}=\sum_{t=1}^{n} \frac{\partial}{\partial \sigma^{2}}\left(-\frac{1}{2 \sigma^{2}}+\frac{1}{2 \sigma^{4}} d\left(y_{t} ; \mu_{t}\right)\right) \\
& =\frac{n}{2 \sigma^{4}}-\frac{1}{\sigma^{6}} \sum_{t=1}^{n} d\left(y_{t} ; \mu_{t}\right) .
\end{aligned}
$$

Sendo $\mathrm{E}\left[d\left(y_{t} ; \mu_{t}\right)\right]=\sigma^{2}($ Propriedade $\mathrm{B} .3)$, temos

$$
\mathrm{E}\left(\frac{\partial^{2} \ell\left(\beta, \sigma^{2}\right)}{\partial \sigma^{4}}\right)=-\frac{n}{2 \sigma^{4}}
$$


Assim, a matriz informação de Fisher para $\left(\beta, \sigma^{2}\right)$ é dada por

$$
K=K\left(\beta, \sigma^{2}\right)=\left(\begin{array}{cc}
K_{\beta \beta} & 0 \\
0 & K_{\sigma^{2} \sigma^{2}}
\end{array}\right),
$$

em que

$$
K_{\beta \beta}=\frac{1}{\sigma^{2}} X^{\top} A X, \quad \text { com } A \text { definido em } 3.11 \text { e } K_{\sigma^{2} \sigma^{2}}=\frac{n}{2 \sigma^{4}}
$$

Quando o tamanho da amostra é grande, sob condições gerais de regularidade ((Sen \& Singer (1993)), a distribuição dos estimadores de máxima verossimilhança é, aproximadamente, dada por

$$
\left(\begin{array}{l}
\widehat{\beta} \\
\widehat{\sigma}^{2}
\end{array}\right) \sim N_{k+1}\left(\left(\begin{array}{l}
\beta \\
\sigma^{2}
\end{array}\right), K^{-1}\right),
$$

onde $\widehat{\beta}$ e $\widehat{\sigma}^{2}$ são, respectivamente, os estimadores de máxima verossimilhança de $\beta$ e $\sigma^{2}$, e $K^{-1}=K^{-1}\left(\beta, \sigma^{2}\right)$ é a inversa da matriz de informação de Fisher definida em (3.12).

\subsubsection{Estimação dos parâmetros}

Os estimadores de máxima verossimilhança dos parâmetros $\beta$ e $\sigma^{2}$ são obtidos através das equações $U_{\beta}\left(\beta, \sigma^{2}\right)=0$ e $U_{\sigma^{2}}\left(\beta, \sigma^{2}\right)=0$. Entretanto, apenas o estimador para $\sigma^{2}$ possui uma forma fechada.

Assim, para estimação de $\beta$, será necessário utilizar algum algoritmo de maximização numérica do logaritmo da função de verossimilhança (definido em 3.3), como o método de Newton-Raphson, escore de Fisher ou quasi-Newton. Nesta seção, descreveremos o método de quasi-Newton.

Denotando $U(\beta)=U_{\beta}\left(\beta, \sigma^{2}\right)^{\top}$, o método de quasi-Newton ou BFGS para estimar $\beta$, de forma semelhante ao descrito no capítulo anterior, é dado por

$$
\beta^{(m+1)}=\beta^{(m)}-\alpha^{(m)} B^{(m)} U\left(\beta^{(m)}\right), \quad m=0,1, \ldots,
$$

em que $\alpha^{(m)}$ é um escalar determinado por algum procedimento de busca linear a partir de $\beta^{(m)}$ na direção $-B^{(m)} U\left(\beta^{(m)}\right)$ de forma que $f\left(y ; \beta^{(m)}\right)$ cresça nessa direção. $B^{(m)}$ são matrizes 
simétricas e positivas definidas, dadas por

$$
B^{(m+1)}=B^{(m)}-\frac{B^{(m)} s^{(m)}\left(s^{(m)}\right)^{\top} B^{(m)}}{\left(s^{(m)}\right)^{\top} B^{(m)} s^{(m)}}+\frac{y^{(m)}\left(y^{(m)}\right)^{\top}}{\left(y^{(m)}\right)^{\top} s^{(m)}}, \quad m=0,1, \ldots,
$$

em que $s^{(m)}=\beta^{(m+1)}-\beta^{(m)}$ e $y^{(m)}=U\left(\beta^{(m+1)}\right)-U\left(\beta^{(m)}\right)$. Mais detalhes sobre este método podem ser encontrados em Nocedal \& Wright (1999).

A partir da equação $U_{\sigma^{2}}\left(\beta, \sigma^{2}\right)=0$, obtemos o estimador de máxima verossimilhança de $\sigma^{2}$ dado por

$$
\hat{\sigma}^{2}=\frac{1}{n} \sum_{t=1}^{n} d\left(y_{t} ; \hat{\mu}_{t}\right)
$$

\subsection{Resíduos}

Nesta seção, apresentamos o resíduo padronizado ponderado 2 para análise do ajuste do modelo de regressão simplex, seguindo o mesmo princípio utilizado por Espinheira et al. (2008) e Ferrari \& Cribari-Neto (2004) para definição desse resíduo no modelo de regressão beta.

O método iterativo scoring de Fisher para estimação de $\beta$ é dado por

$$
\beta^{(m+1)}=\beta^{(m)}+\left(X^{\top} A^{(m)} X\right)^{-1} X^{\top} T^{(m)} u^{(m)}
$$

com $u, T$ e $A$ definidos em (3.6) e (3.11). Podemos reescrever o processo acima como regressão de mínimos quadrados ponderados:

$$
\beta^{(m+1)}=\left(X^{\top} A^{(m)} X\right)^{-1} X^{\top} W^{(m)} z^{(m)},
$$

onde $z^{(m)}=\eta^{(m)}+A^{-1(m)} T^{(m)} u^{(m)}$ e $\eta=\left(\eta_{1}, \ldots, \eta_{n}\right)^{\top}=X \beta$

Na convergência, temos

$$
\widehat{\beta}=\left(X^{\top} \widehat{A} X\right)^{-1} X^{\top} \widehat{A} z \text {, com } z=\widehat{\eta}+\widehat{A}^{-1} \widehat{T} \widehat{u} \text {. }
$$


A matriz $H$ é definida como

$$
H=\widehat{A}^{1 / 2} X\left(X^{\top} \widehat{A} X\right)^{-1} X^{\top} \widehat{A}^{1 / 2}
$$

Considerando $\sigma^{2}$ conhecido, definimos o resíduo padronizado ponderado 2 como

$$
r_{t}^{p p}=\frac{\widehat{u}_{t}}{\sqrt{q_{t}\left(1-h_{t t}\right)}},
$$

em que $\widehat{u}_{t}=-(1 / 2) d^{\prime}\left(y_{t} ; \widehat{\mu}_{t}\right)$, com $d^{\prime}\left(y_{t} ; \mu_{t}\right)$ definido em 3.5 , $h_{t t}$ é o $t$-ésimo elemento da diagonal de $H, q_{t}=\widehat{\operatorname{Var}}\left(u_{t}\right)=\sigma^{2}\left[3 \sigma^{2} /\left(\widehat{\mu}_{t}\left(1-\widehat{\mu}_{t}\right)\right)+1 /\left(\widehat{\mu}_{t}^{3}\left(1-\widehat{\mu}_{t}\right)^{3}\right)\right]$ e $\widehat{\mu}_{t}=g^{-1}\left(x_{t}^{\top} \widehat{\beta}\right)$. Na prática, como $\sigma^{2}$ é desconhecido, substituímos $\sigma^{2}$ pela estimativa de máxima verossimilhança $\widehat{\sigma}^{2}$. 


\section{Capítulo 4}

\section{Algumas medidas de diagnóstico}

\subsection{Introdução}

Uma etapa importante do ajuste de um modelo de regressão é a análise de diagnóstico, pois nos permite verificar possíveis afastamentos das suposições feitas para o modelo e nos auxilia na identificação de observações extremas com alguma interferência desproporcional nos resultados do ajuste.

Neste capítulo apresentamos algumas medidas de diagnóstico construídas a partir de um processo iterativo reponderado para estimar os parâmetros de regressão $\beta$, na modelagem através da regressão beta e da regressão simplex.

O método iterativo scoring de Fisher para estimar $\beta$ é dado por

$$
\beta^{(m+1)}=\beta^{(m)}+\left(X^{\top} P^{(m)} X\right)^{-1} X^{\top} T^{(m)} b^{(m)},
$$

em que $T=\operatorname{diag}\left\{1 / g^{\prime}\left(\mu_{1}\right), \ldots, 1 / g^{\prime}\left(\mu_{n}\right)\right\}, P=W$ e $b=y^{*}-\mu^{*}$, sendo $W, y^{*}$ e $\mu^{*}$ definidos na Seção 2.3.1 para o caso do modelo de regressão beta, ou $P=A$ e $b=u$, para o caso do modelo de regressão simplex, sendo $A$ e $u$ definidos na Seção 3.3.1 .

Podemos reescrever (4.1) como o seguinte processo iterativo de mínimos quadrados reponderados

$$
\beta^{(m+1)}=\left(X^{\top} P^{(m)} X\right)^{-1} X^{\top} P^{(m)} z^{(m)},
$$


em que $z^{(m)}=\eta^{(m)}+P^{-1(m)} T^{(m)} b^{(m)}$, com $\eta=\left(\eta_{1}, \ldots, \eta_{n}\right)^{\top}=X \beta$. Na convergência do processo (4.2), temos

$$
\widehat{\beta}=\left(X^{\top} \hat{P} X\right)^{-1} X^{\top} \hat{P} z,
$$

com $z=\hat{\eta}+\hat{P}^{-1} \hat{T} \hat{b}$, em que $\hat{\eta}, \hat{P}, \hat{T}$ e $\hat{b}$ são as quantidades $\eta, P, T$ e $b$ avaliadas no estimador de máxima verossimilhança. Notemos que $\widehat{\beta}$ em 4.3 pode ser visto como a estimativa de $\beta$ obtida pela regressão de uma variável dependente modificada $z$ com uma matriz de planejamento $X$ e uma matriz de pesos $\hat{P}$.

\subsection{Ponto Alavanca}

Na equação 4.3), $\hat{\beta}$ pode ser interpretado como a solução de mínimos quadrados da regressão normal linear de $\hat{P}^{1 / 2} z$ tendo como matriz de planejamento $\hat{P}^{1 / 2} X$. Dessa forma, o resíduo ordinário, definido como a diferença entre os valores observado e ajustado, é dado por

$$
r_{O}=(I-H) \hat{P}^{1 / 2} z
$$

sendo $I$ a matriz identidade e $H$ uma matriz diagonal dada por

$$
H=\hat{P}^{1 / 2} X\left(X^{\top} \hat{P} X\right)^{-1} X^{\top} \hat{P}^{1 / 2}
$$

Então, considerando que $\hat{P}^{1 / 2} z$ faz o papel do vetor resposta, podemos chamar $H$ de matriz de projeção ortogonal ou matriz chapéu, como na regressão normal linear em que $\hat{P}$ é a identidade. Isso nos leva a utilizar os elementos da diagonal principal de $H, h_{t t}$, para detectarmos a presença de pontos alavancas, assim como Paula (2004) fez para modelos lineares generalizados (MLGs) e Venezuela, Botter \& Sandoval (2007) fizeram para os MLGs com medidas repetidas.

Um ponto alavanca possui um perfil diferente dos demais em relação aos valores das variáveis explicativas. Na prática, construímos um gráfico de $h_{t t}$ versus o índice das observações $t, t=1, \ldots, n$. Um valor grande de $h_{t t}$ comparado com os valores das demais observações pode indicar que um ponto é alavanca.

Supondo que todos os pontos exerçam a mesma influência sobre os valores ajustados, pode-se esperar que cada valor de $h_{t t}$ esteja próximo de $\operatorname{tr}(H) / n=k / n$. Dessa forma, sugerese examinar os pontos para os quais $h_{t t} \geq 2 k / n$, conhecidos como pontos de alavanca ou de 
alto leverage.

Devemos notar que os valores de $h_{t t}$ dependem da matriz de pesos $\hat{P}$, logo observações com valores grandes de $h_{t t}$ nem sempre são pontos alavanca. Assim, os pontos indicados como alavanca precisam ser examinados com cautela.

\subsection{Ponto Aberrante}

Um ponto aberrante é aquele que apresenta perfil diferente das demais obervações em relação aos valores da variável resposta e, também, possui valor baixo na matriz de projeção $H$. Com isso, um ponto dificilmente é alavanca e aberrante.

Graficamente, para detectarmos pontos aberrantes, utilizamos o resíduo padronizado versus o índice das observações $t$. Na verificação do ajuste do modelo de regressão beta, utilizaremos o resíduo ponderado padronizado 2 definido na Seção 2.5.3. No caso do modelo de regressão simplex, será utilizado o resíduo definido na Seção 3.4 .

\subsection{Distância de Cook}

Um ponto influente é aquele que exerce um peso desproporcional nas estimativas dos parâmetros do modelo, possui um perfil diferente dos demais no que tange aos valores da variável resposta e apresenta valor alto na matriz de projeção $H$. Uma medida de influência de cada observação nas estimativas dos parâmetros é a distância de Cook (Cook (1977)) definida como

$$
D C_{t}=\frac{1}{k}\left(\widehat{\beta}-\widehat{\beta}_{(t)}\right)^{\top} X^{\top} \hat{P} X\left(\widehat{\beta}-\widehat{\beta}_{(t)}\right),
$$

em que $\widehat{\beta}_{(t)}$ é a estimativa do parâmetro sem a $t$-ésima observação. Essa medida mede o afastamento entre a estimativa do vetor paramétrico utilizando todas as observações $(\widehat{\beta})$ e sem a observação $y_{t}\left(\widehat{\beta}_{(t)}\right)$.

Uma aproximação para a distância de Cook, para evitar ajustar o modelo $n+1$ vezes, é dada por

$$
D C_{t}=\left(r_{t}^{p p}\right)^{2} \frac{h_{t t}}{k\left(1-h_{t t}\right)}
$$


em que $r_{t}^{p p}$ é o resíduo ponderado padronizado 2 definido na Seção 2.5 .3 no caso do modelo de regressão beta, ou na Seção 3.4 no caso do modelo de regressão simplex, e $k$ é a dimensão de $\beta$.

Usualmente, construímos um gráfico de $D C_{t}$ versus o índice $t$ para detectar pontos suspeitos de serem influentes. Um ponto influente é aquele que apresenta um valor grande na distância de Cook quando comparado com os demais valores. Nota-se que $D C_{t}$ será grande quando o $t$-ésimo ponto fornecer $r_{t}^{p p}$ grande ou quando $h_{t t}$ for próximo de um.

\subsection{Critério de Informação de Akaike (AIC)}

Uma medida utilizada para selecionar o modelo mais parcimonioso entre os ajustados, ou seja, que esteja bem ajustado e com um número reduzido de parâmetros, é o critério de informação de Akaike (AIC).

O critério de informação de Akaike é dado por

$$
A I C=-2 l(\hat{\beta})+2 k,
$$

sendo $l(\hat{\beta})$ o logaritmo da função de verossimilhança avaliado na estimativa de máxima verossimilhança de $\beta, \hat{\beta}$, sob o modelo ajustado e $k$ a dimensão de $\beta$.

O modelo selecionado será o que minimiza a medida AIC dentre o conjunto de modelos ajustados.

\subsection{Gráfico de Probabilidade Meio-Normal com Enve- lope Simulado}

O gráfico de probabilidade meio-normal com envelope simulado é uma ferramenta de diagnóstico muito útil para avaliarmos o ajuste do modelo.

Este gráfico é construído baseado nos resíduos padronizados. Detalhes sobre sua construção podem ser encontrados em Neter, Kutner, Naschtheim \& Wasserman (1996). O envelope 
simulado é a banda de confiança. A ocorrência de pontos próximos ou fora da banda de confiança indicam que o modelo não está apropriado.

Neste trabalho, utilizaremos os resíduos ponderados padronizados 2, definidos nas Seções 2.5.3 e 3.4 para a construção dos gráficos de probabilidade meio-normal com envelope simulado para os modelos de regressão beta e simplex, respectivamente. 


\section{Capítulo 5}

\section{Aplicações}

Neste capítulo aplicamos os modelos de regressão beta e simplex a dois conjuntos de dados, avaliando-os por meio das medidas de diagnótico apresentadas no Capítulo 3. Os conjuntos de dados encontram-se no Apêndice C. Alguns programas utilizados para as análises dos dados encontram-se no Apêndice D.

\subsection{Aplicação I: Dados de Gasolina}

Nesta aplicação, analisamos os dados de gasolina de Prater (1956). O conjunto de dados contém 32 observações. A variável dependente (y) é a proporção de petróleo convertida em gasolina depois do processo de destilação e fracionamento. As variáveis independentes são: tipo do petróleo e temperatura $\left({ }^{\circ} \mathrm{F}\right)$ em que toda a gasolina se evapora. Há dez diferentes tipos de petróleo que, sob diferentes condições de destilação, são obtidos pela combinação de outras variáveis: gravidade do petróleo (graus API), pressão do vapor do petróleo (Ibf/in²), temperatura em que $10 \%$ do petróleo é vaporizado.

Especificamos o modelo para a resposta média usando um intercepto $\left(x_{0}=1\right)$, nove variáveis dummy $\left(x_{1}, \ldots, x_{9}\right)$ para os primeiros nove níveis do tipo do petróleo e a temperatura $\left(x_{10}\right)$ em que toda gasolina é vaporizada. 


\section{Ajuste do Modelo de Regressão Beta}

Consideremos que as observações $y_{1}, \ldots, y_{32}$ são independentes e têm distribuição beta com média $\mu_{t},(t=1, \ldots, 32)$, e parâmetro de precisão $\phi$ desconhecido. O modelo para as médias é escrito da seguinte forma

$$
g\left(\mu_{t}\right)=\beta_{0}+\beta_{1} x_{t 1}+\ldots+\beta_{10} x_{t 10} .
$$

Ajustamos o modelo de regressão beta especificado em (5.1) utilizando a função de ligação logito. Os resultados da estimação são dados na Tabela 5.1. Observa-se que todas as variáveis são significantes para o modelo.

Tabela 5.1: Estimativas dos parâmetros do modelo de regressão beta ajustado para os dados de gasolina.

\begin{tabular}{cccc}
\hline & & Erro & \\
Parâmetro & Estimativa & Padrão & p-valor \\
\hline$\beta_{0}$ & $-6,1596$ & 0,1823 & 0,0000 \\
$\beta_{1}$ & 1,7277 & 0,1012 & 0,0000 \\
$\beta_{2}$ & 1,3226 & 0,1179 & 0,0000 \\
$\beta_{3}$ & 1,5723 & 0,1161 & 0,0000 \\
$\beta_{4}$ & 1,0597 & 0,1024 & 0,0000 \\
$\beta_{5}$ & 1,1338 & 0,1035 & 0,0000 \\
$\beta_{6}$ & 1,0402 & 0,1060 & 0,0000 \\
$\beta_{7}$ & 0,5437 & 0,1091 & 0,0000 \\
$\beta_{8}$ & 0,4959 & 0,1089 & 0,0000 \\
$\beta_{9}$ & 0,3858 & 0,1186 & 0,0011 \\
$\beta_{10}$ & 0,0110 & 0,0004 & 0,0000 \\
$\phi$ & 440,2783 & 110,0256 & \\
\hline
\end{tabular}

Em seguida, calculamos as medidas de diagnóstico para verificar se há algum ponto com comportamento atípico. A Figura 5.1 não indica pontos de alavanca. Apenas a observação 4 aparece como possível ponto aberrante e influente (Figuras $5.1 \mathrm{~b}-\mathrm{c}$ ).

O gráfico de probabilidade meio-normal com envelope simulado é apresentado na Figura 5.1 d. Observamos que todos os pontos se encontram dentro da banda de confiança. 


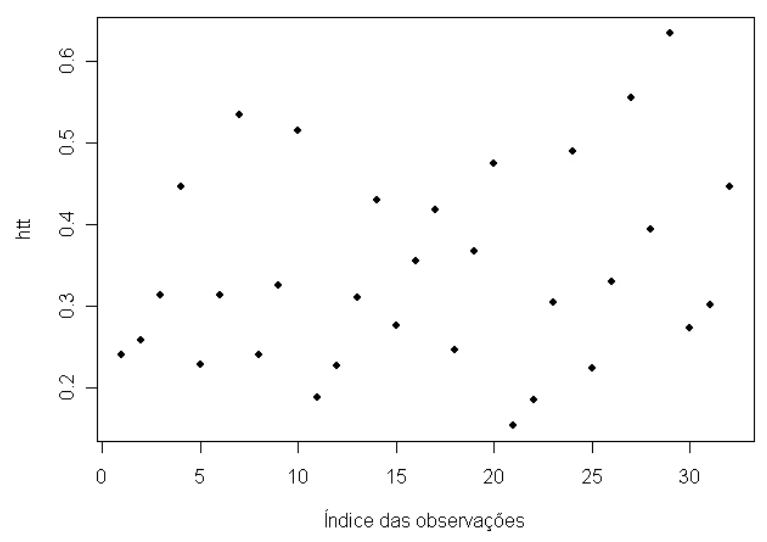

(a)

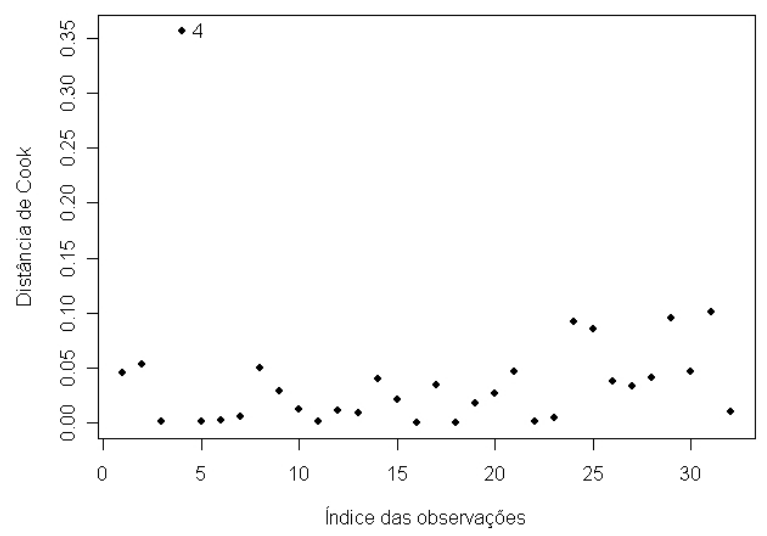

(c)

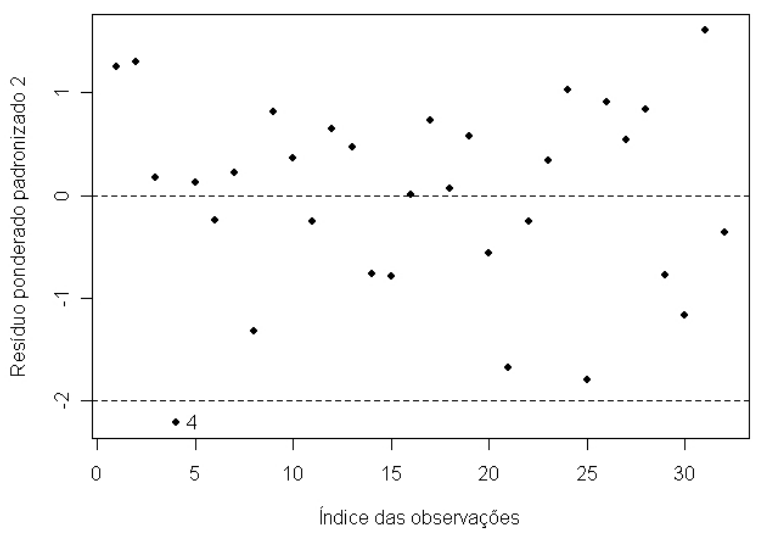

(b)

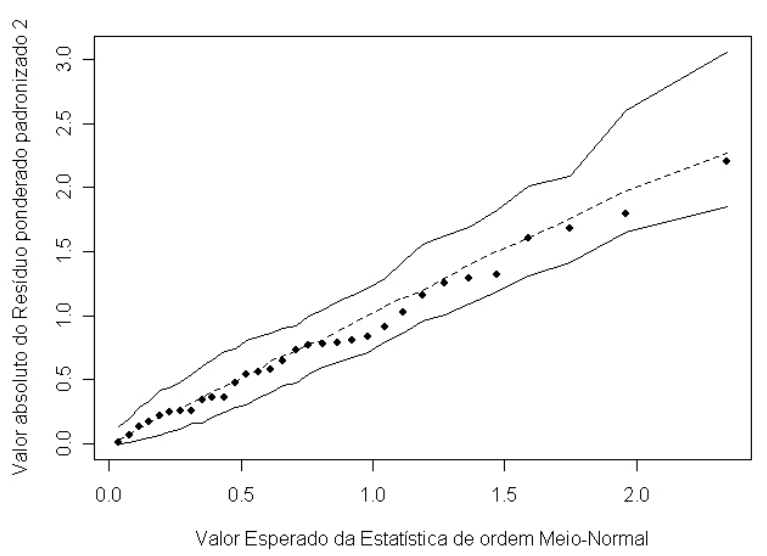

(d)

Figura 5.1: Gráficos de diagnóstico do modelo de regressão beta ajustado para os dados de gasolina.

Para avaliar a influência que as observações com características distintas das demais (destacadas como alavanca, aberrante e/ou influente) têm sobre as estimativas dos parâmetros de regressão e dispersão, vamos utilizar a seguinte medida

$$
\text { Variação Percentual }=\frac{\hat{\theta}_{-p o n t o(s)}-\hat{\theta}}{\hat{\theta}} \times 100 \% \text {, }
$$


em que $\hat{\theta}_{-p o n t o(s)}$ é a estimativa do parâmetro $\theta$ sem o(s) ponto(s) com característica(s) distinta(s) dos demais e $\hat{\theta}$ é a estimativa de $\theta$ com todos os pontos no modelo. Essa medida verifica, descritivamente, o quanto variam as estimativas dos parâmetros na ausência do(s) ponto(s) com comportamento(s) distintos dos demais.

Ajustamos novamente o modelo de regressão beta, excluindo a observação 4. A Tabela 5.2 apresenta as estimativas e as variações percentuais dos parâmetros desse ajuste. Como temos $\mathrm{n}=32$ observações, isto implica que cada unidade experimental deve influenciar em 3,13\% nas estimativas dos parâmetros. Podemos notar que não há grande alteração nas estimativas dos $\beta$ 's. A maior variação ocorre com o parâmetro de precisão $(\phi)$, cuja estimativa aumentou 31, 23\%. No geral, a eliminação da observação 4 não muda as conclusões inferenciais. Assim, o modelo de regressão beta ajustado com a observação 4 é adequado aos dados.

Tabela 5.2: Variação percentual das estimativas dos parâmetros do modelo de regressão beta ajustado para os dados de gasolina, sem a observação 4 .

\begin{tabular}{cccc}
\hline & Todas obs & \multicolumn{2}{c}{ Sem obs 4} \\
Parâmetro & Estimativa & Estimativa & Var.Percentual \\
\hline$\beta_{0}$ & $-6,1596$ & $-6,3565$ & $3,20 \%$ \\
$\beta_{1}$ & 1,7277 & 1,8869 & $9,21 \%$ \\
$\beta_{2}$ & 1,3226 & 1,3704 & $3,61 \%$ \\
$\beta_{3}$ & 1,5723 & 1,6251 & $3,36 \%$ \\
$\beta_{4}$ & 1,0597 & 1,0807 & $1,98 \%$ \\
$\beta_{5}$ & 1,1338 & 1,1516 & $1,57 \%$ \\
$\beta_{6}$ & 1,0402 & 1,0577 & $1,68 \%$ \\
$\beta_{7}$ & 0,5437 & 0,5652 & $3,95 \%$ \\
$\beta_{8}$ & 0,4959 & 0,5007 & $0,97 \%$ \\
$\beta_{9}$ & 0,3858 & 0,3852 & $-0,16 \%$ \\
$\beta_{10}$ & 0,0110 & 0,0115 & $4,55 \%$ \\
$\phi$ & 440,2783 & 577,7907 & $31,23 \%$ \\
\hline
\end{tabular}




\section{Ajuste do Modelo de Regressão Simplex}

Aplicamos agora uma nova modelagem para os dados de gasolina, considerando que as observações $y_{t}, t=1, \ldots, 32$ são independentes e têm distribuição simplex com média $\mu_{t} \mathrm{e}$ parâmetro de dispersão $\sigma^{2}$ desconhecido. O modelo para as médias é dado por

$$
g\left(\mu_{t}\right)=\beta_{0}+\beta_{1} x_{t 1}+\ldots+\beta_{10} x_{t 10}
$$

A Tabela 5.3 mostra os resultados do ajuste do modelo de regressão simplex dado em (5.2) para os dados de gasolina, utilizando a função de ligação logito. Obtemos as mesmas conclusões do ajuste do modelo de regressão beta, ou seja, que todas as variáveis são significantes para o modelo.

Tabela 5.3: Estimativas dos parâmetros do modelo de regressão simplex ajustado para os dados de gasolina.

\begin{tabular}{cccc}
\hline & \multicolumn{3}{c}{ Erro } \\
Parâmetro & Estimativa & Padrão & p-valor \\
\hline$\beta_{0}$ & $-6,6544$ & 0,2156 & 0,0000 \\
$\beta_{1}$ & 1,9669 & 0,1413 & 0,0000 \\
$\beta_{2}$ & 1,4764 & 0,1433 & 0,0000 \\
$\beta_{3}$ & 1,6443 & 0,1465 & 0,0000 \\
$\beta_{4}$ & 1,1605 & 0,1308 & 0,0000 \\
$\beta_{5}$ & 1,1420 & 0,1458 & 0,0000 \\
$\beta_{6}$ & 1,1267 & 0,1407 & 0,0000 \\
$\beta_{7}$ & 0,4396 & 0,1214 & 0,0003 \\
$\beta_{8}$ & 0,3785 & 0,1274 & 0,0030 \\
$\beta_{9}$ & 0,4404 & 0,1553 & 0,0046 \\
$\beta_{10}$ & 0,0123 & 0,0005 & 0,0000 \\
$\sigma^{2}$ & 0,2253 & 0,1187 & \\
\hline
\end{tabular}

Fazendo a análise de diagnóstico, pela Figura 5.2 a não observamos pontos de alavanca. Podemos verificar que os pontos 1, 4, 21, 25 e 31 se destacam como possíveis pontos aberrantes (Figura 5.2b). Os pontos 1, 21 e 25 também são considerados possíveis pontos influentes (Figura 5.2 c). Na Figura 5.2d, nota-se que alguns pontos estão fora da banda de confiança, indicando que o modelo não está bem ajustado. 


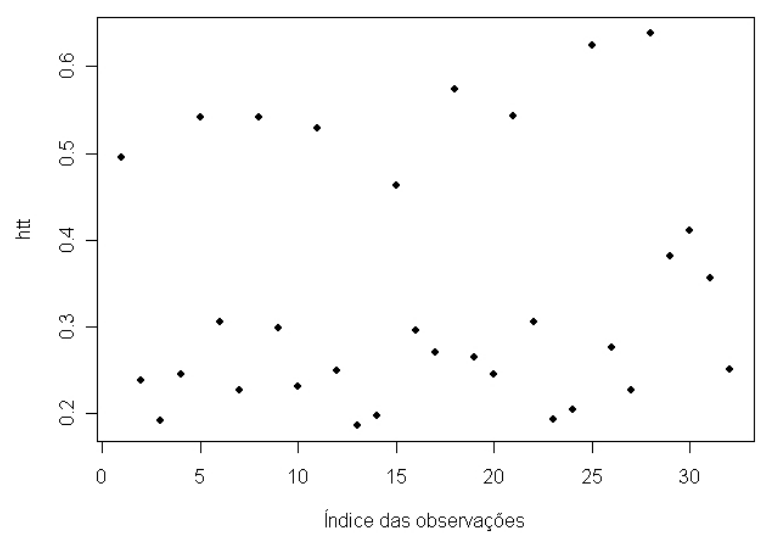

(a)

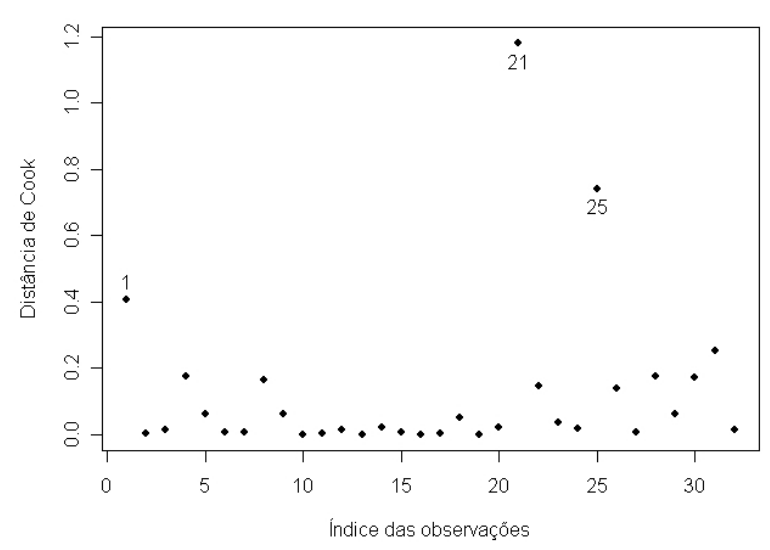

(c)

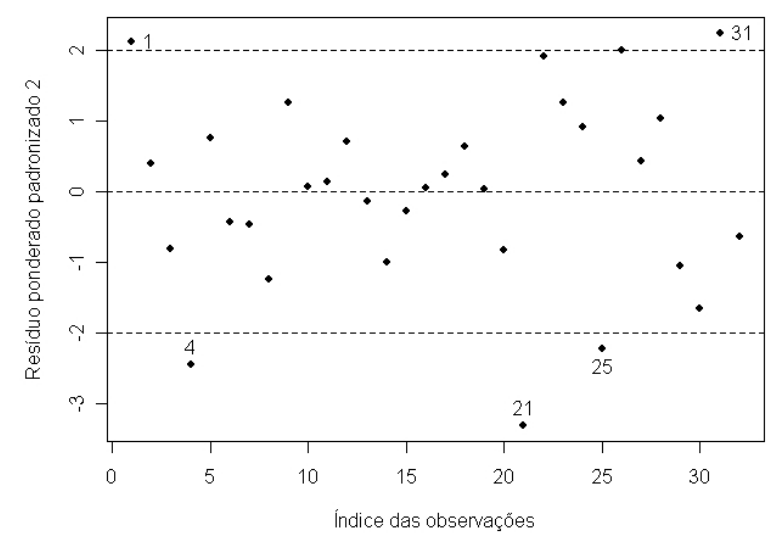

(b)

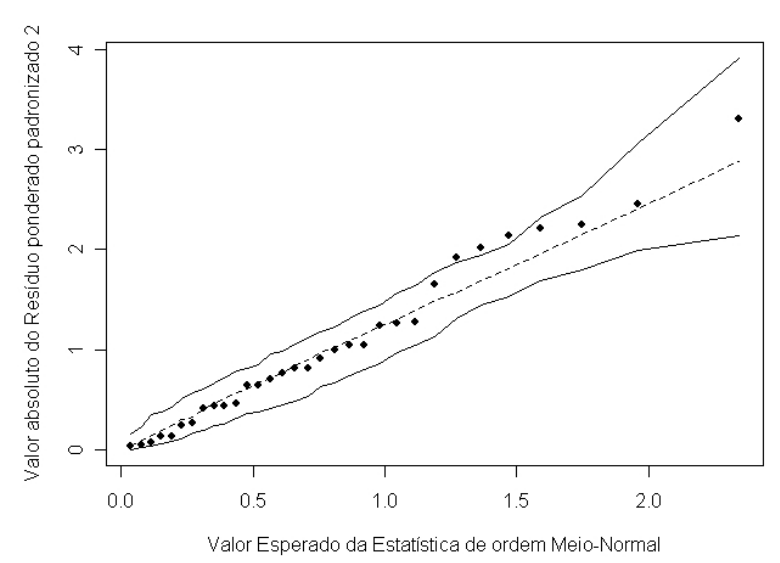

(d)

Figura 5.2: Gráficos de diagnóstico do modelo de regressão simplex ajustado para os dados de gasolina.

Vamos analisar a influência das observações 1, 4, 21, 25 e 31 nas estimativas dos parâmetros, com o ajuste do modelo de regressão simplex excluindo tais pontos, separadamente. As Tabelas 5.4 e 5.5 mostram as estimativas dos parâmetros e as respectivas variações percentuais desses ajustes. As estimativas dos parâmetros não se alteram muito sem a observação 1. Notamos que a maior variação sem a observação 4 ocorre na estimativa do parâmetro de dispersão $\sigma^{2}(-14,67 \%)$, mas a exclusão da observação 21 causa maior variação $(-33,78 \%)$ na estimativa desse parâmetro. As variações percentuais nas estimativas de $\beta_{7}$ são maiores retirando-se os pontos 31 e 21. A estimativa de $\beta_{8}$ muda bastante excluindo-se o ponto 
25 (aumenta 52,12\%), seguido pela exclusão do 31 (aumenta 43,92\%). A eliminação da observação 31 causa as maiores variações nas estimativas dos demais parâmetros.

Tabela 5.4: Variação percentual nas estimativas dos parâmetros do modelo de regressão simplex ajustado para os dados de gasolina, sem as observações 1 e 4.

\begin{tabular}{cccccc}
\hline & Todas obs & \multicolumn{2}{c}{ Sem obs 1 } & \multicolumn{2}{c}{ Sem obs 4 } \\
Parâmetro & Estimativa & Estimativa & Var.Percentual & Estimativa & Var.Percentual \\
\hline$\beta_{0}$ & $-6,6544$ & $-6,8097$ & $2,33 \%$ & $-6,8234$ & $2,54 \%$ \\
$\beta_{1}$ & 1,9669 & 1,8460 & $-6,15 \%$ & 2,1160 & $7,58 \%$ \\
$\beta_{2}$ & 1,4764 & 1,5295 & $3,60 \%$ & 1,5343 & $3,92 \%$ \\
$\beta_{3}$ & 1,6443 & 1,6953 & $3,10 \%$ & 1,6998 & $3,38 \%$ \\
$\beta_{4}$ & 1,1605 & 1,1984 & $3,27 \%$ & 1,2018 & $3,56 \%$ \\
$\beta_{5}$ & 1,1420 & 1,1569 & $1,3 \%$ & 1,1582 & $1,42 \%$ \\
$\beta_{6}$ & 1,1267 & 1,1556 & $2,57 \%$ & 1,1581 & $2,79 \%$ \\
$\beta_{7}$ & 0,4396 & 0,4779 & $8,71 \%$ & 0,4813 & $9,49 \%$ \\
$\beta_{8}$ & 0,3785 & 0,3960 & $4,62 \%$ & 0,3976 & $5,05 \%$ \\
$\beta_{9}$ & 0,4404 & 0,4453 & $1,11 \%$ & 0,4458 & $1,23 \%$ \\
$\beta_{10}$ & 0,0123 & 0,0127 & $3,25 \%$ & 0,0127 & $3,25 \%$ \\
$\sigma^{2}$ & 0,2253 & 0,2030 & $-9,90 \%$ & 0,1918 & $-14,87 \%$ \\
\hline
\end{tabular}

Tabela 5.5: Variação percentual nas estimativas dos parâmetros do modelo de regressão simplex ajustado para os dados de gasolina, sem as observações 21,25 e 31.

\begin{tabular}{cccccccc}
\hline & Todas obs & \multicolumn{2}{c}{ Sem obs 21 } & \multicolumn{2}{c}{ Sem obs 25 } & \multicolumn{2}{c}{ Sem obs 31 } \\
Parâmetro & Estimativa & Estimativa & Var.Perc. & Estimativa & Var.Perc. & Estimativa & Var.Perc. \\
\hline$\beta_{0}$ & $-6,6544$ & $-6,4046$ & $-3,75 \%$ & $-6,5142$ & $-2,11 \%$ & $-6,8311$ & $2,66 \%$ \\
$\beta_{1}$ & 1,9669 & 1,8948 & $-3,67 \%$ & 1,9264 & $-2,06 \%$ & 2,1345 & $8,52 \%$ \\
$\beta_{2}$ & 1,4764 & 1,3921 & $-5,71 \%$ & 1,4289 & $-3,22 \%$ & 1,6447 & $11,40 \%$ \\
$\beta_{3}$ & 1,6443 & 1,5634 & $-4,92 \%$ & 1,5987 & $-2,77 \%$ & 1,8124 & $10,22 \%$ \\
$\beta_{4}$ & 1,1605 & 1,1011 & $-5,12 \%$ & 1,1270 & $-2,89 \%$ & 1,3275 & $14,39 \%$ \\
$\beta_{5}$ & 1,1420 & 1,1185 & $-2,06 \%$ & 1,1287 & $-1,16 \%$ & 1,3071 & $14,46 \%$ \\
$\beta_{6}$ & 1,1267 & 1,0816 & $-4,00 \%$ & 1,1012 & $-2,26 \%$ & 1,2930 & $14,76 \%$ \\
$\beta_{7}$ & 0,4396 & 0,5640 & $28,30 \%$ & 0,4060 & $-7,64 \%$ & 0,6066 & $37,99 \%$ \\
$\beta_{8}$ & 0,3785 & 0,3524 & $-6,90 \%$ & 0,5748 & $51,86 \%$ & 0,5438 & $43,67 \%$ \\
$\beta_{9}$ & 0,4404 & 0,4327 & $-1,75 \%$ & 0,4361 & $-0,98 \%$ & 0,6047 & $37,31 \%$ \\
$\beta_{10}$ & 0,0123 & 0,0116 & $-5,69 \%$ & 0,0119 & $-3,25 \%$ & 0,0123 & $0,00 \%$ \\
$\sigma^{2}$ & 0,2253 & 0,1492 & $-33,78 \%$ & 0,1975 & $-12,34 \%$ & 0,1981 & $-12,07 \%$ \\
\hline
\end{tabular}

Foram feitos outros ajustes do modelo de regressão simplex, sem os pontos 1, 4, 21, 25 
e 31, combinados de diversas formas, porém as análises de diagnósticos ainda não foram satisfatórias para indicar a adequação de algum dos modelos. Por fim, ajustamos o modelo de regressão simplex retirando os pontos $(1,4,21,25$ e 31). Os resultados desse ajuste são apresentados na Tabela 5.6 .

Tabela 5.6: Variação percentual nas estimativas dos parâmetros do modelo de regressão simplex ajustado para os dados de gasolina, sem as observações $(1,4,21,25$ e 31$)$.

\begin{tabular}{cccc}
\hline & Todas obs & \multicolumn{2}{c}{ Sem obs $(1,4,21,25$, e 31) } \\
Parâmetro & Estimativa & Estimativa & Var.Percentual \\
\hline$\beta_{0}$ & $-6,6544$ & $-6,5880$ & $-1,00 \%$ \\
$\beta_{1}$ & 1,9669 & 2,0498 & $4,21 \%$ \\
$\beta_{2}$ & 1,4764 & 1,5605 & $5,70 \%$ \\
$\beta_{3}$ & 1,6443 & 1,7315 & $5,30 \%$ \\
$\beta_{4}$ & 1,1605 & 1,2673 & $9,20 \%$ \\
$\beta_{5}$ & 1,1420 & 1,2814 & $12,21 \%$ \\
$\beta_{6}$ & 1,1267 & 1,2465 & $10,63 \%$ \\
$\beta_{7}$ & 0,4396 & 0,7284 & $65,70 \%$ \\
$\beta_{8}$ & 0,3785 & 0,7374 & $94,82 \%$ \\
$\beta_{9}$ & 0,4404 & 0,5943 & $34,95 \%$ \\
$\beta_{10}$ & 0,0123 & 0,0117 & $-4,88 \%$ \\
$\sigma^{2}$ & 0,2253 & 0,0493 & $-78,12 \%$ \\
\hline
\end{tabular}

Aplicamos novamente as técnicas de diagnóstico para detectar outros possíveis pontos de alavanca, aberrantes e/ou influentes. A Figura 5.3 a não apresenta pontos de alavanca. Os pontos 8 e 9 aparecem como possíveis pontos aberrantes na Figura 5.3b. Na Figura 5.3c, observamos que a distância de Cook dos pontos 8 e 28 são maiores que as demais observações, indicando serem possíveis pontos influentes.

Como os pontos 8, 9 e 28 se destacaram na análise de diagnóstico do modelo de regressão simplex ajustado sem as observações $(1,4,21,25$ e 31), vamos verificar se tais pontos têm alguma influência nas estimativas dos parâmetros da regressão. Para isso, ajustamos novamente o modelo de regressão simplex, retirando também esses três pontos.

A Tabela 5.7 apresenta as estimativas dos parâmetros do modelo ajustado sem as observações $(1,4,8,9,21,25,28$ e 31) e as respectivas variações percentuais dessas estimativas em relação às estimativas do modelo ajustado sem as observações (1, 4, 21, 25 e 31). Podemos 


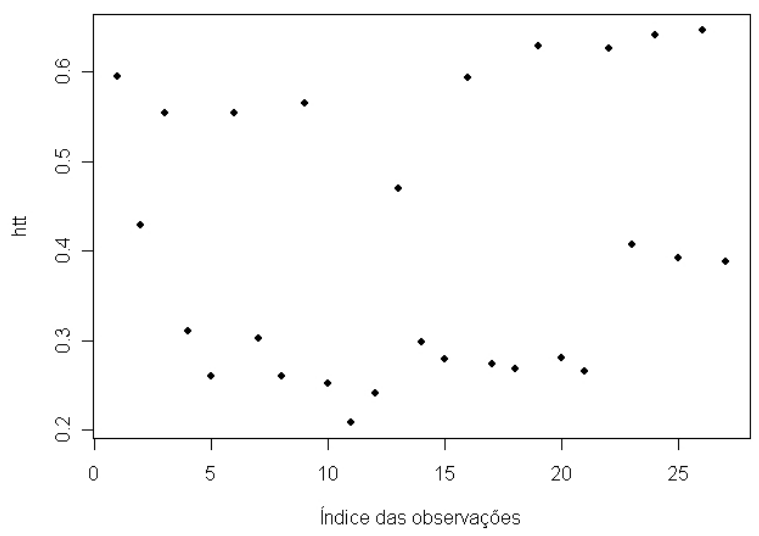

(a)

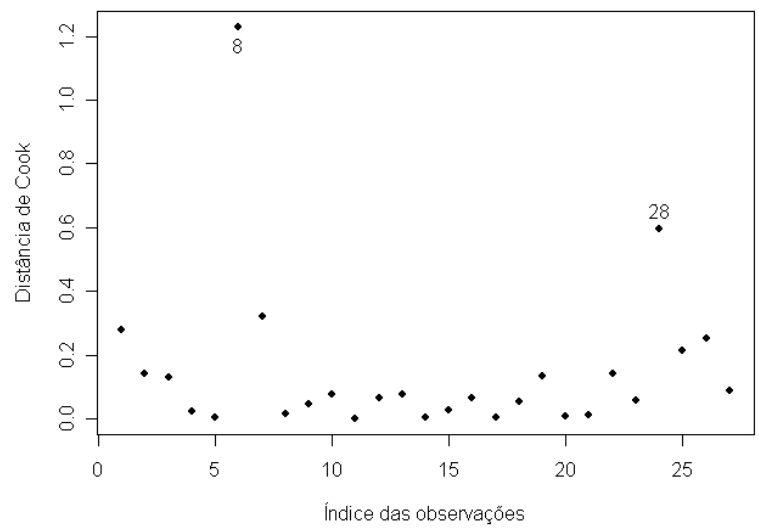

(c)

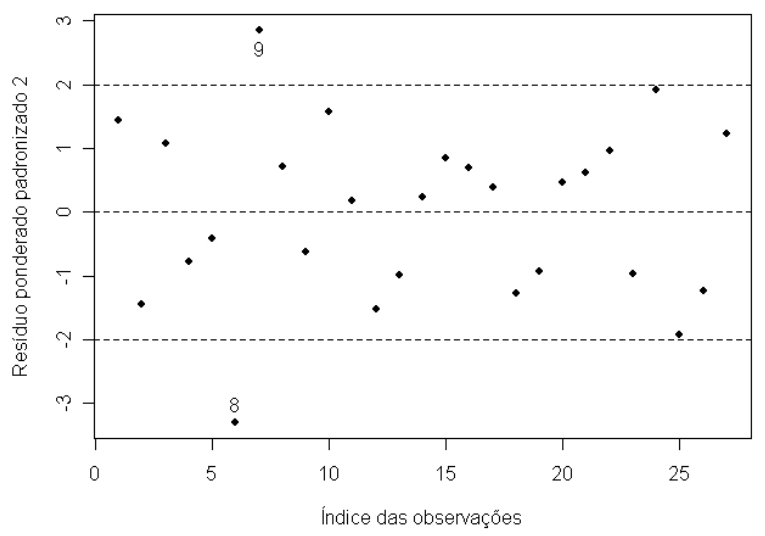

(b)

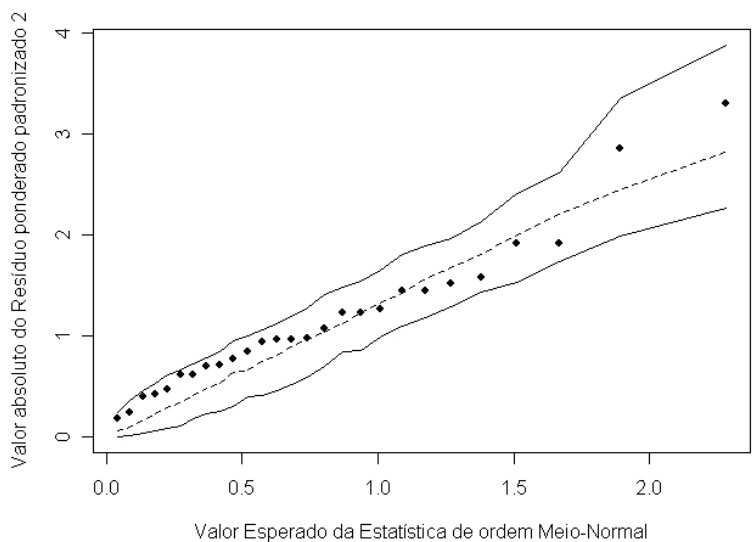

(d)

Figura 5.3: Gráficos de diagnóstico do modelo de regressão simplex ajustado para os dados de gasolina, sem as observações $(1,4,21,25$ e 31).

observar que as estimativas não se alteram muito, exceto para os parâmetros $\beta_{9}$ e $\sigma^{2}$. No entanto, excluídas as observações $(1,4,21,25$ e 31), a eliminação das observações 8,9 e 28 não muda as conclusões inferenciais.

A Figura 5.3d mostra o gráfico de probabilidade meio-normal. Observamos que os pontos estão dentro da banda de confiança, logo o modelo de regressão simplex ajustado sem as observações $(1,4,21,25$ e 31$)$ é adequado aos dados. 
Tabela 5.7: Variação percentual nas estimativas dos parâmetros do modelo de regressão simplex ajustado para os dados de gasolina, sem as observações $(1,4,8,9,21,25,28$ e 31).

\begin{tabular}{cccc}
\hline & Sem as observações & \multicolumn{2}{c}{ Sem as observações } \\
Parâmetro & Estimativa & Estimativa & Var.Percentual \\
\hline$\beta_{0}$ & $-6,5880$ & $-6,5240$ & $-0,97 \%$ \\
$\beta_{1}$ & 2,0498 & 2,0352 & $-0,71 \%$ \\
$\beta_{2}$ & 1,5605 & 1,5385 & $-1,41 \%$ \\
$\beta_{3}$ & 1,7315 & 1,7843 & $3,05 \%$ \\
$\beta_{4}$ & 1,2673 & 1,2517 & $-1,23 \%$ \\
$\beta_{5}$ & 1,2814 & 1,2747 & $-0,52 \%$ \\
$\beta_{6}$ & 1,2465 & 1,2345 & $-0,96 \%$ \\
$\beta_{7}$ & 0,7284 & 0,7176 & $-1,48 \%$ \\
$\beta_{8}$ & 0,7374 & 0,7378 & $0,05 \%$ \\
$\beta_{9}$ & 0,5943 & 0,4493 & $-24,40 \%$ \\
$\beta_{10}$ & 0,0117 & 0,0115 & $-1,71 \%$ \\
$\sigma^{2}$ & 0,0493 & 0,0253 & $-48,68 \%$ \\
\hline
\end{tabular}

Pelo critério de informação de Akaike, obtemos $A I C=-147,60$ para o modelo de regressão beta ajustado com todas as observações e $A I C=-140,89$ para o modelo de regressão simplex ajustado sem as observações (1, 4, 21, 25 e 31). Logo, podemos concluir que o modelo de regressão beta é o mais adequado para explicar a proporção de petróleo convertida em gasolina, pois apresenta o menor valor do $A I C$.

\subsection{Aplicação II: Dados de Oxidação de Amônia}

Nesta aplicação, utilizamos os dados de oxidação de amônia, analisados originalmente por Brownlee (1965). Este estudo consiste em analisar a perda na conversão da amônia em ácido nítrico, em 21 dias de processos de produção de ácido nítrico em uma planta industrial. O ácido nítrico é utilizado na produção de fertilizantes, corantes, medicamentos, etc. O processo ocorre da seguinte forma: o gás amônia reage com o oxigêno do ar formando o óxido nítrico, que reage novamente com o oxigênio do ar produzindo o dióxido de nitrogênio. Este, por sua vez, reage com a água formando o ácido nítrico e também o óxido nítrico. Então, o óxido 
nítrico produzido é absorvido e reutilizado no processo. Há liberação de muito calor em todas essas reações químicas, sendo necessário o resfriamento do processo através da água.

A variável dependente $(y)$ é a perda na conversão de amônia em ácido nítrico, que corresponde à proporção de amônia não convertida em ácido nítrico. O objetivo é modelar a proporção de amônia não convertida em ácido nítrico em função das covariáveis corrente de ar $\left(x_{1}\right)$, temperatura da água $\left(x_{2}\right)$ utilizada no resfriamento do processo e a concentração de ácido nítrico $\left(x_{3}\right)$.

\section{Ajuste do Modelo de Regressão Beta}

Para ajustar o modelo de regressão beta a esses dados, assumiremos que $y_{1}, \ldots, y_{21}$ são independentes e seguem uma distribuição beta com média $\mu_{t}, t=1, \ldots, 21$, e parâmetro de precisão $\phi$ desconhecido. Inicialmente, modelamos a média como

$$
g\left(\mu_{t}\right)=\beta_{0}+\beta_{1} x_{t 1}+\beta_{2} x_{t 2}+\beta_{3} x_{t 3}
$$

sendo $\beta_{0}$ correspondente ao intercepto e $g\left(\mu_{t}\right)$ a função de ligação logito.

Os resultados do ajuste do modelo de regressão beta especificado em (5.3) são dados na Tabela 5.8 .

Tabela 5.8: Estimativas dos parâmetros do modelo de regressão beta ajustado para os dados de oxidação de amônia.

\begin{tabular}{cccc}
\hline & \multicolumn{3}{c}{ Erro } \\
Parâmetro & Estimativa & Padrão & p-valor \\
\hline$\beta_{0}$ & $-7,7297$ & 0,6795 & 0,0000 \\
$\beta_{1}$ & 0,0294 & 0,0063 & 0,0000 \\
$\beta_{2}$ & 0,0741 & 0,0189 & 0,0001 \\
$\beta_{3}$ & 0,0029 & 0,0086 & 0,7390 \\
$\phi$ & 2361,3550 & 730,3160 & \\
\hline
\end{tabular}

Aplicando as técnicas de diagnóstico, construímos os gráficos apresentados nas Figuras 5.4 a-d. A observação 2 aparece como possível ponto de alavanca e a observação 4 como possível ponto aberrante. A distância de Cook das observações 2, 4 e 21 são maiores que as demais observações, destacando-as como possíveis pontos influentes. 
A Figura 5.4d apresenta o gráfico de probabilidade meio-normal. Notamos que os pontos encontram-se dentro da banda de confiança.

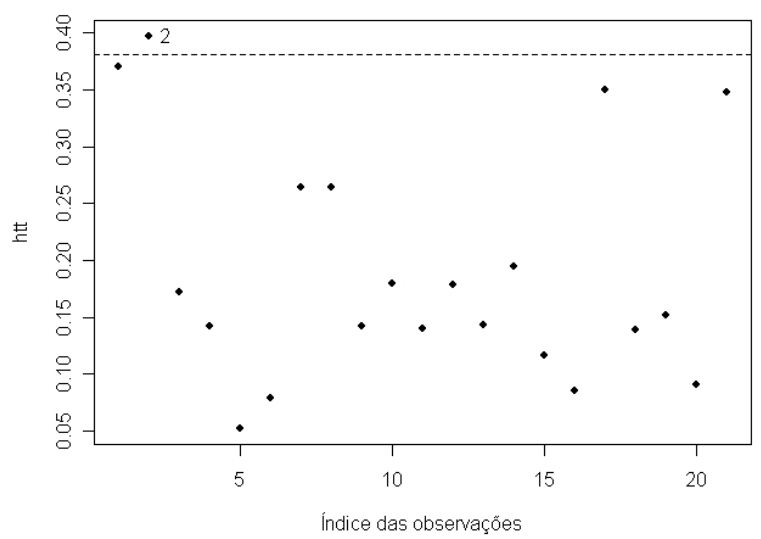

(a)

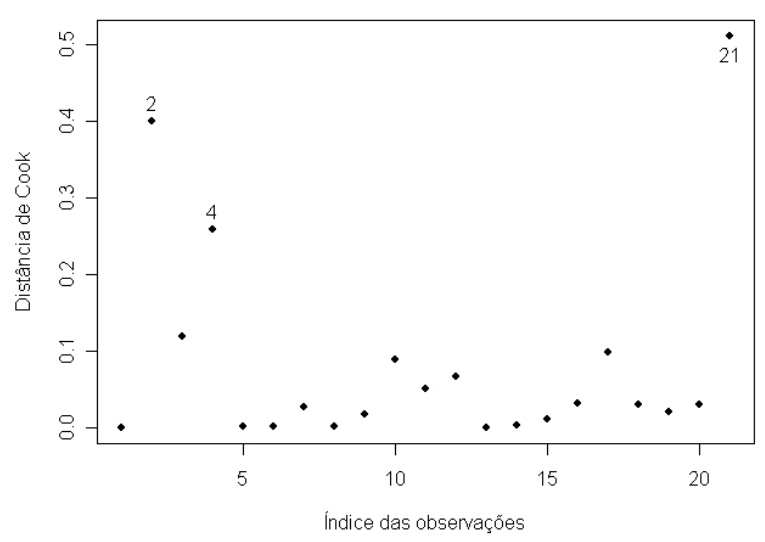

(c)

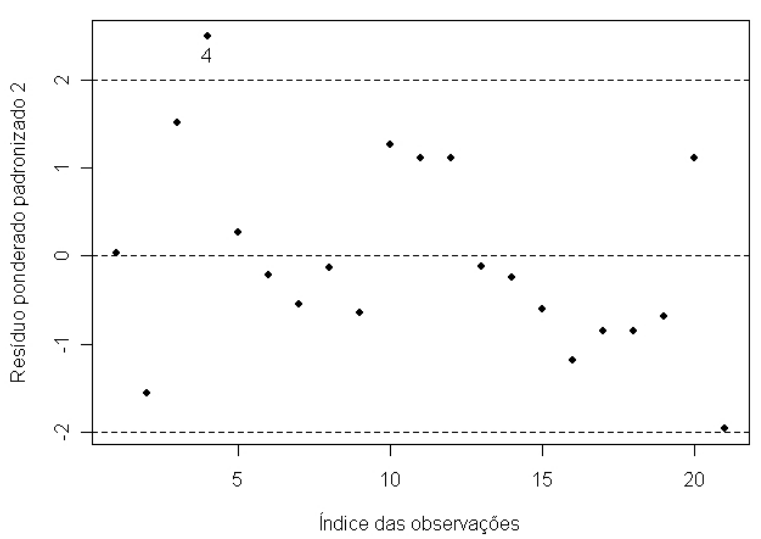

(b)

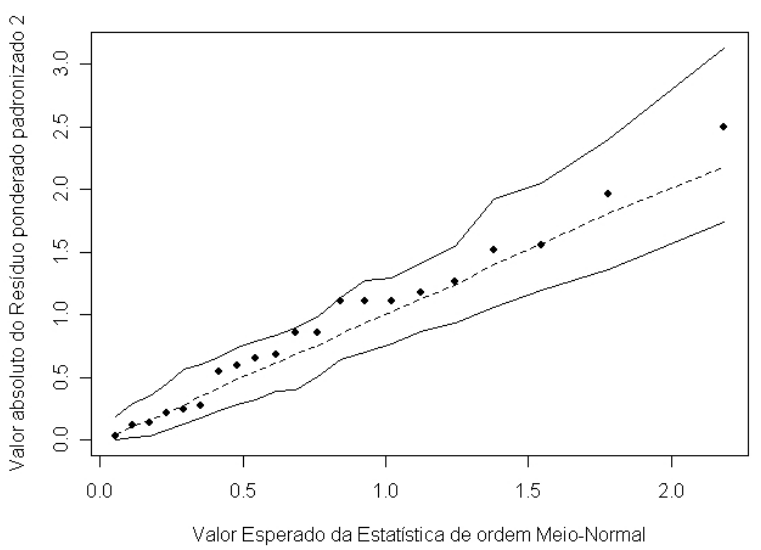

(d)

Figura 5.4: Gráficos de diagnóstico do modelo de regressão beta ajustado para os dados de oxidação de amônia.

Para avaliar se os pontos 2, 4 e 21 exercem alguma influência nas estimativas dos parâmetros, tentamos ajustar novamente o modelo de regressão beta com a exclusão de cada um desses pontos, separadamente. Porém, não há convergência do processo de estimação dos parâmetros. Ajustamos, então, o modelo excluindo esses três pontos. A Tabela 5.9 apresenta as estimativas e as variações percentuais dos parâmetros desse ajuste. Notamos que a exclusão das observações (2, 4 e 21) altera bastante as estimativas dos parâmetros. 
Tabela 5.9: Variação percentual das estimativas dos parâmetros do modelo de regressão beta ajustado para os dados de oxidação de amônia, sem as observações $(2,4$ e 21).

\begin{tabular}{|c|c|c|c|c|}
\hline \multicolumn{4}{|c|}{ Erro } & Variação \\
\hline Parâmetro & Estimativa & Padrão & p-valor & Percentual \\
\hline$\beta_{0}$ & $-7,9636$ & 0,4234 & 0,0000 & $3,03 \%$ \\
\hline$\beta_{1}$ & 0,0465 & 0,0047 & 0,0000 & $58,16 \%$ \\
\hline$\beta_{2}$ & 0,0301 & 0,0140 & 0,0310 & $-59,38 \%$ \\
\hline$\beta_{3}$ & 0,0044 & 0,0055 & 0,4297 & $51,72 \%$ \\
\hline$\phi$ & 6637,8620 & 2214,4630 & & $181,1 \%$ \\
\hline
\end{tabular}

Realizamos nova análise de diagnóstico para verificar se ainda há alguma observação atípica. Pelas Figuras 5.5a-c, apenas a observação 1 aparece como um possível ponto de alavanca, aberrante e influente. Na Figura 5.5d, notamos que alguns pontos estão sobre ou próximos da banda de confiança.

Ajustamos novamente o modelo de regressão beta retirando também a observação 1, para verificar se essa observação exerce influência nas estimativas dos parâmentos do modelo ajustado sem as observações (2, 4 e 21). A Tabela 5.10 mostra as estimativas desse ajuste. As variações percentuais dessas estimativas em relação às estimativas obtidas com a exclusão das observações (2, 4 e 21) são dadas na Tabela 5.11. Observamos que, retiradas as observações (2, 4 e 21), a exclusão da observação 1 causa alterações nas estimativas.

Tabela 5.10: Estimativas dos parâmetros do modelo de regressão beta ajustado para os dados de oxidação de amônia, sem as observações (1, 2, 4 e 21).

\begin{tabular}{cccc}
\hline & \multicolumn{3}{c}{ Erro } \\
Parâmetro & Estimativa & Padrão & p-valor \\
\hline$\beta_{0}$ & $-8,0139$ & 0,3692 & 0,0000 \\
$\beta_{1}$ & 0,0529 & 0,0047 & 0,0000 \\
$\beta_{2}$ & 0,0326 & 0,0122 & 0,0076 \\
$\beta_{3}$ & 0,0000 & 0,0051 & 0,9926 \\
$\phi$ & 8734,8850 & 2998,0410 & \\
\hline
\end{tabular}




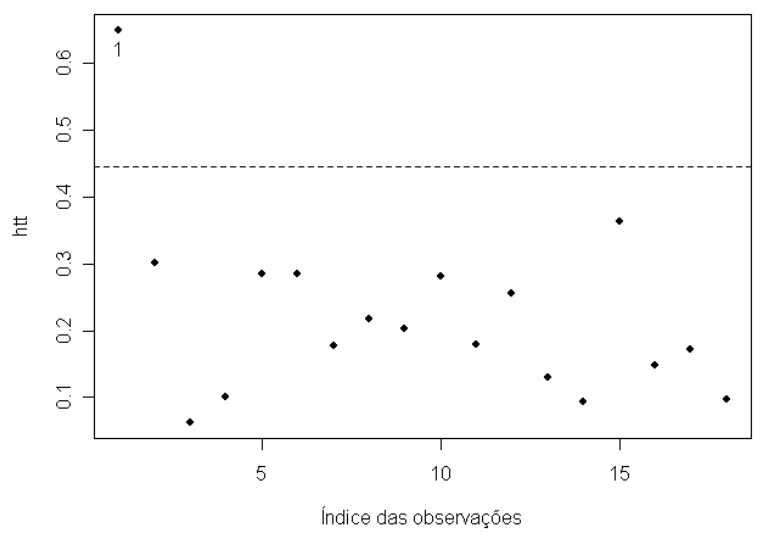

(a)

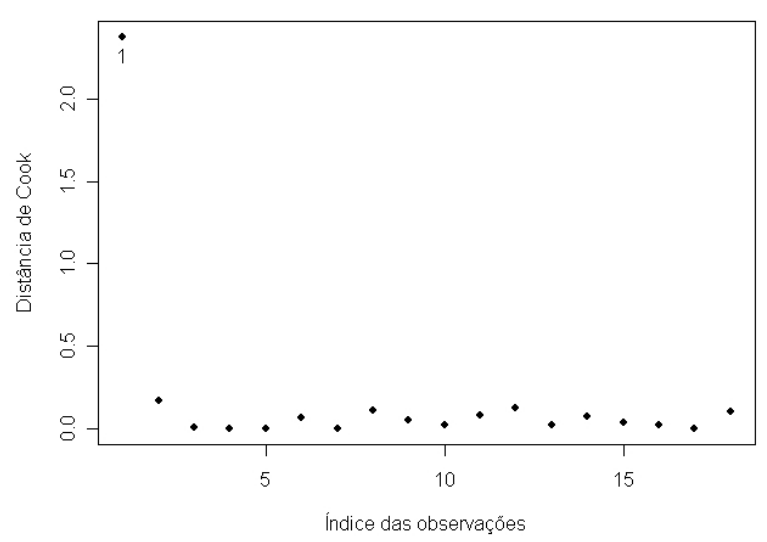

(c)

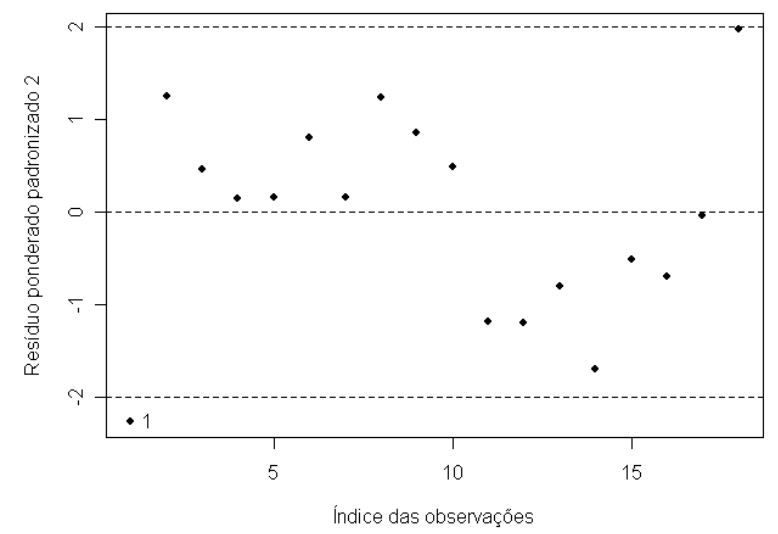

(b)

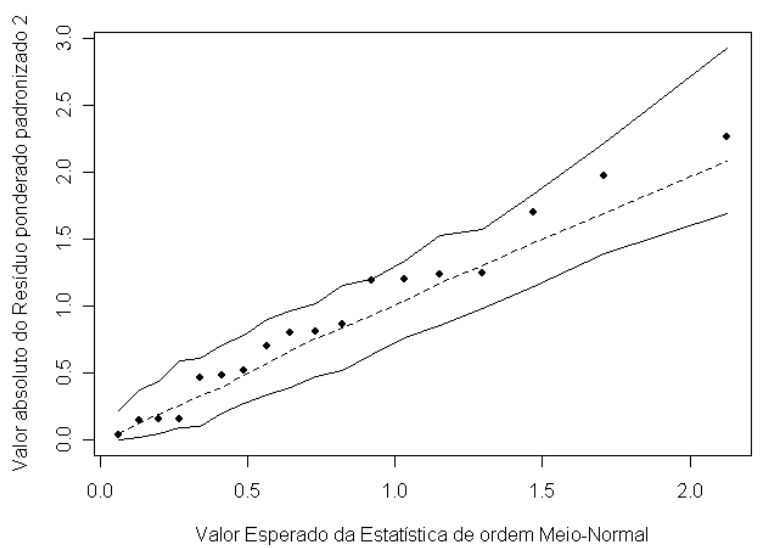

(d)

Figura 5.5: Gráficos de diagnóstico do modelo de regressão beta ajustado para os dados de oxidação de amônia, sem as observações $(2,4$ e 21$)$.

Nosso próximo passo é calcular as medidas de diagnóstico para o modelo de regressão beta ajustado sem as observações (1, 2, 4 e 21). A observação 3 aparece como um possível ponto de alavanca e influente nas Figuras5.6a e 5.6c. A Figura 5.6b destaca a observação 20 como possível ponto aberrante. Na Figura 5.6 d, observamos que todos os pontos estão dentro da banda de confiança.

Em seguida, excluídas as observações (1, 2, 4 e 21), analisamos a variação das estimativas dos parâmetros quando retiramos também as observações 3 e 20. Para isso, ajustamos o 
Tabela 5.11: Variação percentual das estimativas dos parâmetros do modelo de regressão beta ajustado para os dados de oxidação de amônia, sem as observações (1, 2, 4 e 21).

\begin{tabular}{cccc}
\hline & Sem obs (2, 4 e 21) & \multicolumn{2}{c}{ Sem obs $(1,2,4$ e 21) } \\
Parâmetro & Estimativa & Estimativa & Var.Percentual \\
\hline$\beta_{0}$ & $-7,9636$ & $-8,0139$ & $0,63 \%$ \\
$\beta_{1}$ & 0,0465 & 0,0529 & $13,76 \%$ \\
$\beta_{2}$ & 0,0301 & 0,0326 & $8,31 \%$ \\
$\beta_{3}$ & 0,0044 & 0,0000 & $-100,00 \%$ \\
$\phi$ & 6637,8620 & 8734,8850 & $31,59 \%$ \\
\hline
\end{tabular}

modelo de regressão beta excluindo essas observações. A Tabela 5.12 apresenta os resultados dessa análise. Notamos que não há muitas alterações nas estimativas. Apenas a exclusão da observação 3 altera a estimativa de $\beta_{1}$ e a exclusão da observação 20 varia a estimativa de $\phi$. No geral, a eliminação dessas duas observações não mudam as conclusões inferenciais do modelo ajustado sem as observações (1, 2, 4 e 21). Note que a variável concentração de ácido nítrico é não significativa no modelo (Tabela 5.10).

Portanto, o modelo de regressão beta sem as observações (1, 2, 4 e 21) é adequado aos dados de oxidação de amônia e a variável concentração de ácido nítrico não é significativa para explicar a perda na conversão de amônia em ácido nítrico.

Tabela 5.12: Variação percentual das estimativas dos parâmetros do modelo de regressão beta ajustado para os dados de oxidação de amônia, sem as observações 1, 2, 3, 4, 20 e 21.

\begin{tabular}{|c|c|c|c|c|c|}
\hline \multirow[b]{2}{*}{ Parâmetro } & \multirow{2}{*}{$\begin{array}{c}\text { Sem as observações } \\
(1,2,4 \text { e } 21) \\
\text { Estimativa }\end{array}$} & \multicolumn{2}{|c|}{$\begin{array}{c}\text { Sem as observações } \\
(1,2,3,4 \text { e } 21)\end{array}$} & \multicolumn{2}{|c|}{$\begin{array}{c}\text { Sem as observações } \\
(1,2,4,20 \text { e } 21)\end{array}$} \\
\hline & & Estimativa & Var.Perc. & Estimativa & Var.Perc. \\
\hline$\beta_{0}$ & $-8,0139$ & $-8,1917$ & $2,22 \%$ & $-8,2215$ & $2,59 \%$ \\
\hline$\beta_{1}$ & 0,0529 & 0,0613 & $15,88 \%$ & 0,0534 & $0,95 \%$ \\
\hline$\beta_{2}$ & 0,0326 & 0,0307 & $-5,83 \%$ & 0,0316 & $-3,07 \%$ \\
\hline$\beta_{3}$ & 0,0000 & $-0,0030$ & - & 0,0022 & - \\
\hline$\phi$ & 8734,8850 & 8926,0490 & $2,19 \%$ & 11863,0000 & $35,81 \%$ \\
\hline
\end{tabular}




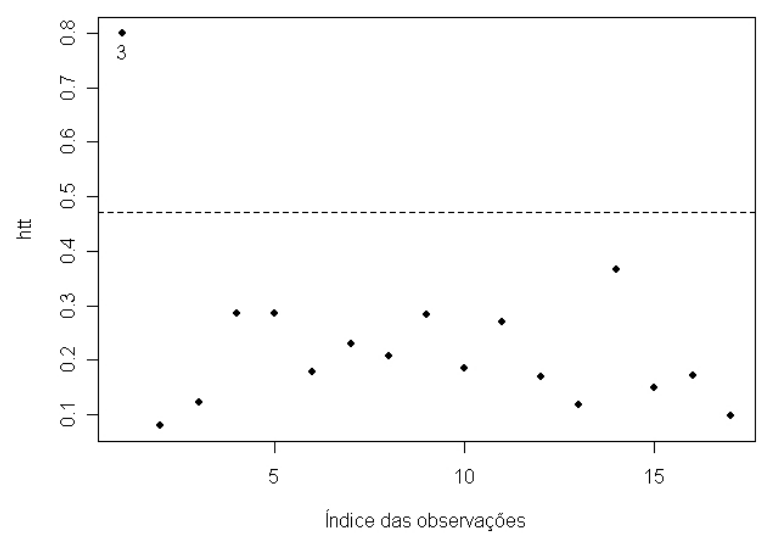

(a)

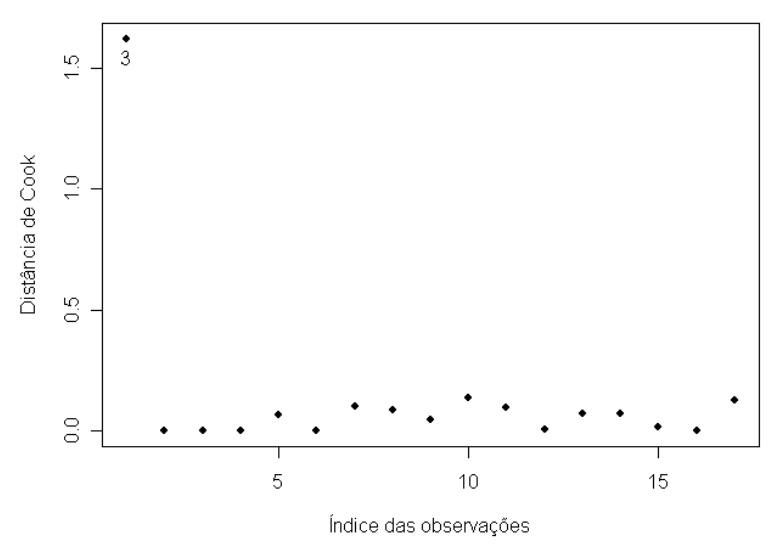

(c)

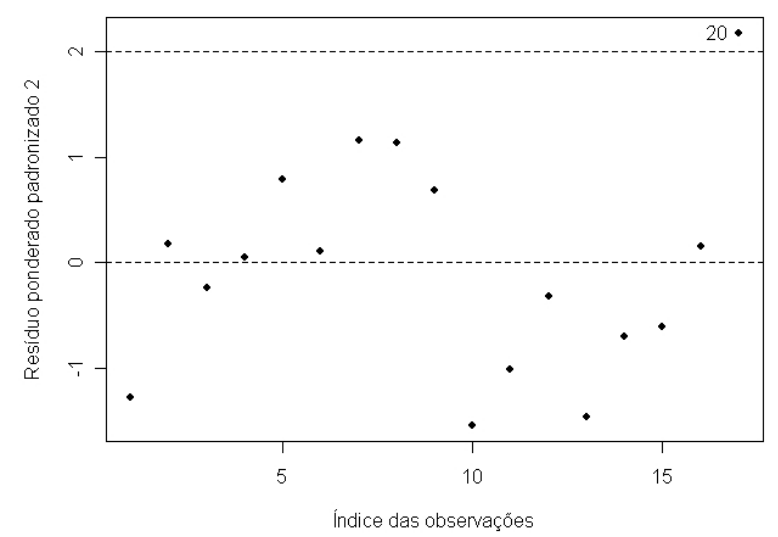

(b)

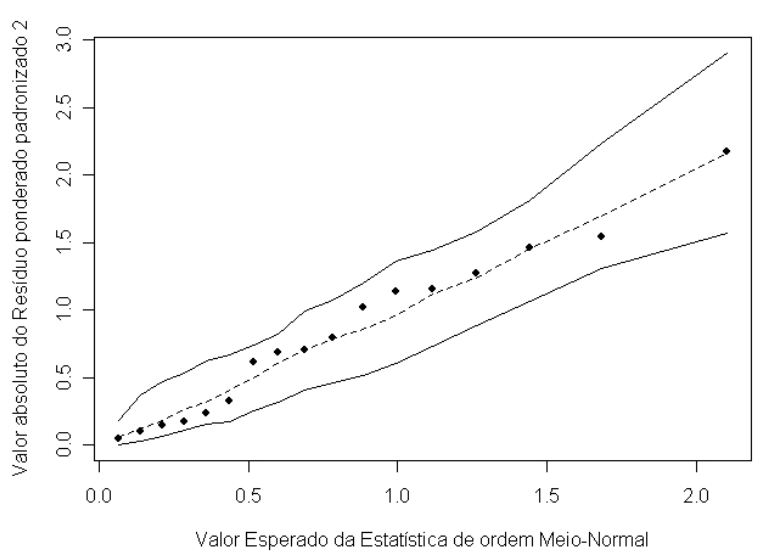

(d)

Figura 5.6: Gráficos de diagnóstico do modelo de regressão beta ajustado para os dados de oxidação de amônia, sem as observações (1, 2, 4 e 21).

\section{Ajuste do Modelo de Regressão Simplex}

Consideremos que $y_{t}, t=1, \ldots, y_{21}$ são independentes e seguem uma distribuição simplex com média $\mu_{t}$ e parâmetro de dispersão $\sigma^{2}$ desconhecido. Utilizando a função de ligação logito, a média é modelada como

$$
g\left(\mu_{t}\right)=\beta_{0}+\beta_{1} x_{t 1}+\beta_{2} x_{t 2}+\beta_{3} x_{t 3},
$$


em que $\beta_{0}$ corresponde ao intercepto.

A Tabela 5.13 mostra as estimativas do ajuste do modelo de regressão simplex especificado em (5.4) aplicado aos dados de oxidação de amônia.

Tabela 5.13: Estimativas dos parâmetros do modelo de regressão simplex ajustado para os dados de oxidação de amônia.

\begin{tabular}{cccc}
\hline & \multicolumn{3}{c}{ Erro } \\
Parâmetro & Estimativa & Padrão & p-valor \\
\hline$\beta_{0}$ & $-8,0837$ & 0,5130 & 0,0000 \\
$\beta_{1}$ & 0,0462 & 0,0071 & 0,0000 \\
$\beta_{2}$ & 0,0521 & 0,0179 & 0,0036 \\
$\beta_{3}$ & 0,0006 & 0,0068 & 0,9234 \\
$\sigma^{2}$ & 1,6783 & 0,3998 & \\
\hline
\end{tabular}

Aplicando as técnicas de diagnóstico, observamos que o ponto 17 aparece como um possível ponto de alavanca (Figura 5.7 a). A Figura 5.7b mostra os pontos 4 e 21 como pontos suspeitos de aberrantes. Os pontos 4, 17 e 21 também se destacam na Figura 5.7 como possíveis pontos influentes. No gráfico de probabilidade meio-normal apresentada na Figura 5.7d, verificamos que há alguns pontos fora ou em cima da banda de confiança, indicando que o modelo não está bem ajustado.

Analisamos agora se os pontos 4, 17 e 21 causam alguma influência nas estimativas dos parâmetros. Ajustamos novamente o modelo de regressão simplex, excluindo-se esses pontos, separadamente. A Tabela 5.14 mostra as estimativas desses ajustes e as respectivas variações percentuais. A exclusão da observação 17 modifica bastante apenas a estimativa de $\beta_{3}$. Notamos que as estimativas dos demais parâmetros se alteram bastante com a eliminação das observações 4 ou 21.

Em seguida, retiramos os pontos $(4,17$ e 21) e ajustamos o modelo. A Tabela 5.15 mostra que a exclusão desses três pontos alteram muito as estimativas dos parâmetros. 


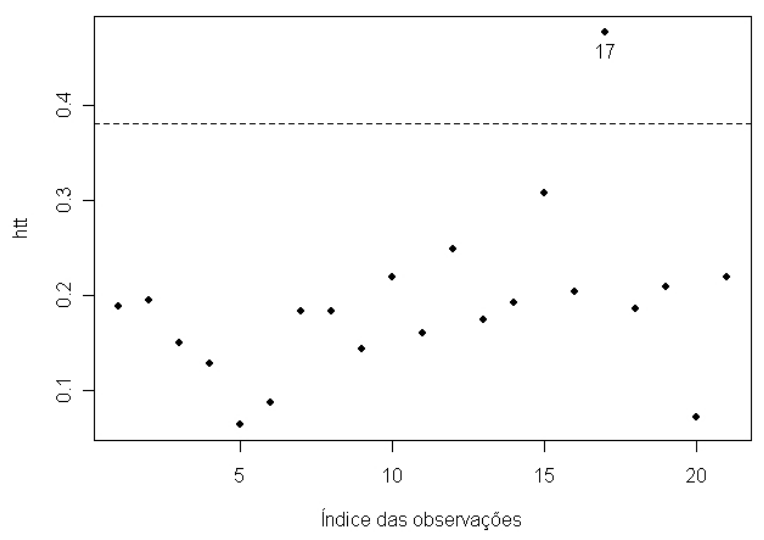

(a)

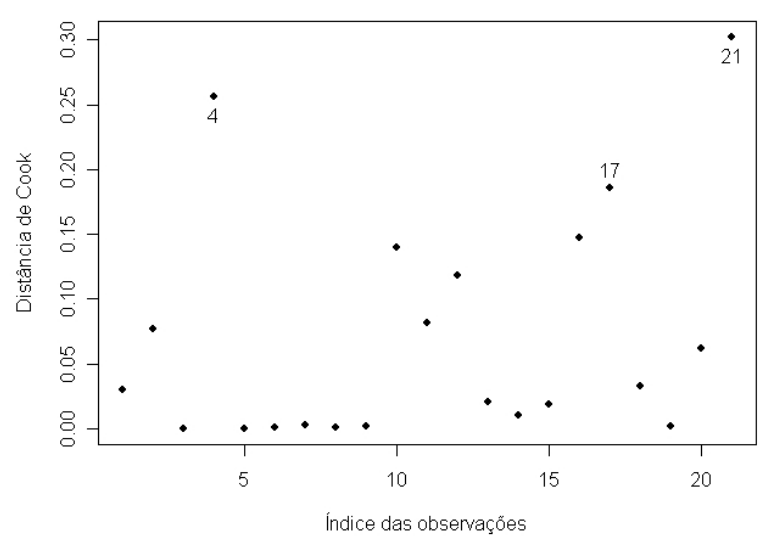

(c)

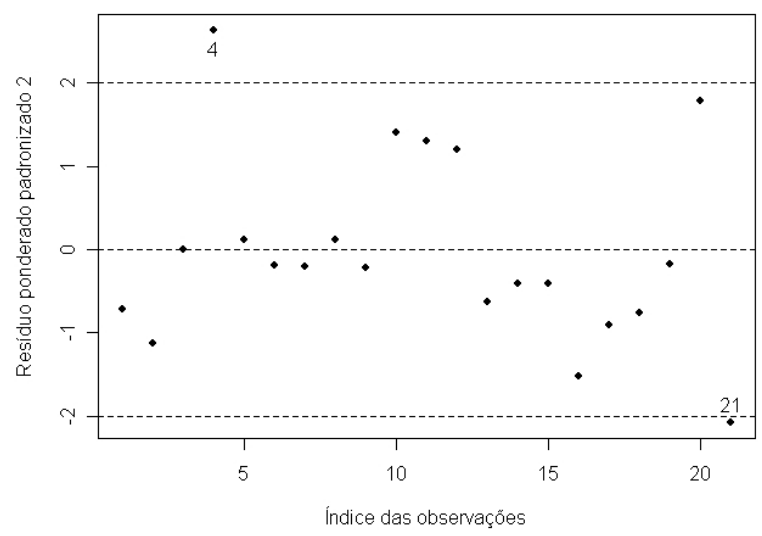

(b)

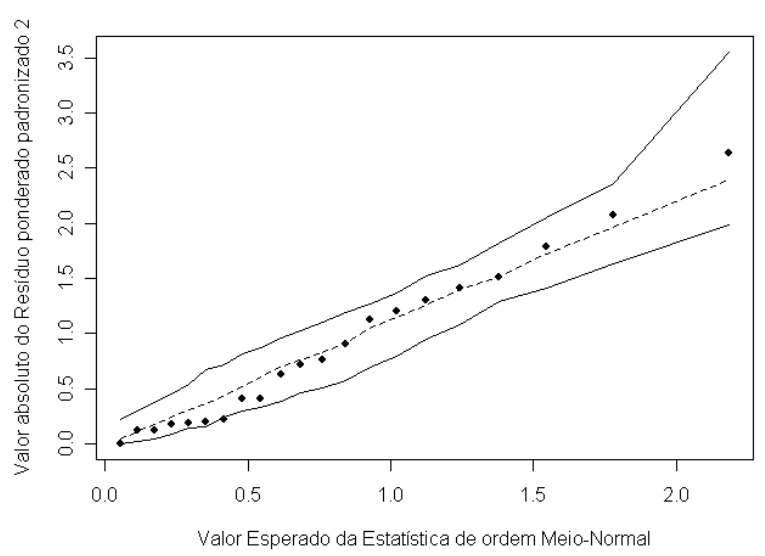

(d)

Figura 5.7: Gráficos de diagnóstico do modelo de regressão simplex ajustado para os dados de oxidação de amônia.

Os gráficos de diagnóstico para o ajuste do modelo sem os pontos (4, 17 e 21) estão apresentados na Figura 5.8. Não há indicativos de pontos suspeitos de alavanca. O ponto 20 aparece como possível ponto aberrante, e o ponto 16 como possível ponto influente. No gráfico de probabilidade meio normal, os pontos estão dentro da banda de confiança. 
Tabela 5.14: Variação percentual das estimativas dos parâmetros do modelo de regressão simplex ajustado para os dados de oxidação de amônia, sem as observações 4, 17 e 21 .

\begin{tabular}{cccccccc}
\hline & Todas obs & \multicolumn{2}{c}{ Sem obs 4} & \multicolumn{2}{c}{ Sem obs 17 } & \multicolumn{2}{c}{ Sem obs 21 } \\
Parâmetro & Estim. & Estim. & Var.Perc. & Estim. & Var.Perc. & Estim. & Var.Perc. \\
\hline$\beta_{0}$ & $-8,0837$ & $-7,9029$ & $-2,24 \%$ & $-7,6870$ & $-4,91 \%$ & $-8,2356$ & $1,88 \%$ \\
$\beta_{1}$ & 0,0462 & 0,0472 & $2,16 \%$ & 0,0458 & $-0,87 \%$ & 0,0535 & $15,8 \%$ \\
$\beta_{2}$ & 0,0521 & 0,0388 & $-25,53 \%$ & 0,0539 & $3,45 \%$ & 0,0409 & $-21,5 \%$ \\
$\beta_{3}$ & 0,0006 & 0,0007 & $16,67 \%$ & $-0,0041$ & $-783,33 \%$ & 0,0003 & $-50,0 \%$ \\
$\sigma^{2}$ & 1,6783 & 1,3291 & $-20,81 \%$ & 1,6905 & $0,73 \%$ & 1,2532 & $-25,33 \%$ \\
\hline
\end{tabular}

Tabela 5.15: Variação percentual das estimativas dos parâmetros do modelo de regressão simplex ajustado para os dados de oxidação de amônia, sem as observações (4, 17 e 21).

\begin{tabular}{cccc}
\hline & Todas obs & \multicolumn{2}{c}{ Sem obs $(4,17$ e 21$)$} \\
Parâmetro & Estimativa & Estimativa & Var.Percentual \\
\hline$\beta_{0}$ & $-8,0837$ & $-7,7995$ & $-3,52 \%$ \\
$\beta_{1}$ & 0,0462 & 0,0539 & $16,67 \%$ \\
$\beta_{2}$ & 0,0521 & 0,0289 & $-44,53 \%$ \\
$\beta_{3}$ & 0,0006 & $-0,0024$ & $-500,00 \%$ \\
$\sigma^{2}$ & 1,6783 & 0,8928 & $-46,80 \%$ \\
\hline
\end{tabular}

O próximo passo é ajustar o modelo de regressão simplex, excluindo também as observações 16 e 20. Calculando as variações das estimativas do modelo ajustado sem os pontos (4, 16, 17, 20 e 21) em relação às estimativas obtidas sem os pontos (4, 17 e 21), verificamos que ainda há grandes alterações nas estimativas de $\beta_{3}$ e $\sigma^{2}$ (Tabela 5.16).

Tabela 5.16: Variação percentual das estimativas dos parâmetros do modelo de regressão simplex ajustado para os dados de oxidação de amônia, sem as observações (4, 16, 17, 20 e 21).

\begin{tabular}{cccc}
\hline & Sem obs (4, 17 e 21) & \multicolumn{2}{c}{ Sem obs (4, 16, 17, 20 e 21) } \\
Parâmetro & Estimativa & Estimativa & Var.Percentual \\
\hline$\beta_{0}$ & $-7,7995$ & $-8,0448$ & $3,15 \%$ \\
$\beta_{1}$ & 0,0539 & 0,0502 & $-6,86 \%$ \\
$\beta_{2}$ & 0,0289 & 0,0263 & $-9,00 \%$ \\
$\beta_{3}$ & $-0,0024$ & 0,0035 & $-245,83 \%$ \\
$\sigma^{2}$ & 0,8928 & 0,4937 & $-44,7 \%$ \\
\hline
\end{tabular}




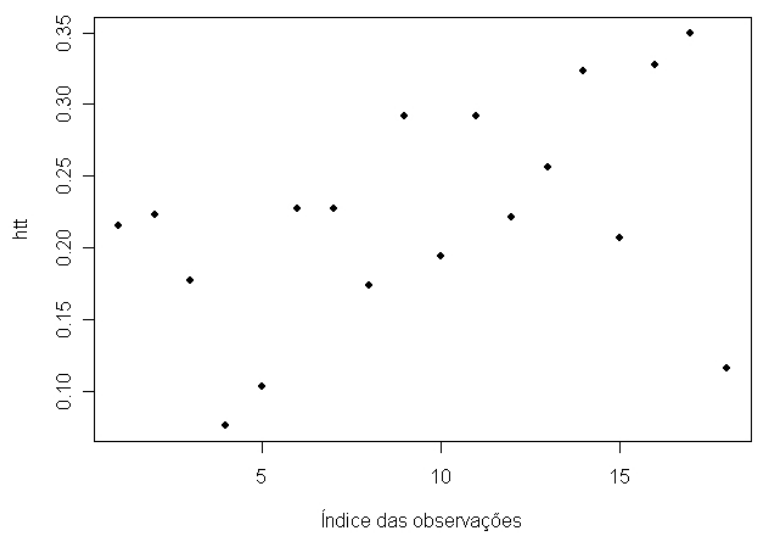

(a)

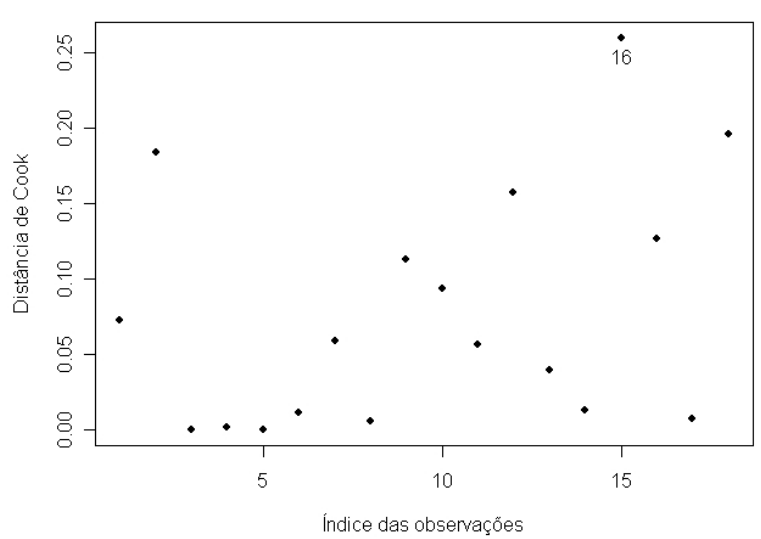

(c)

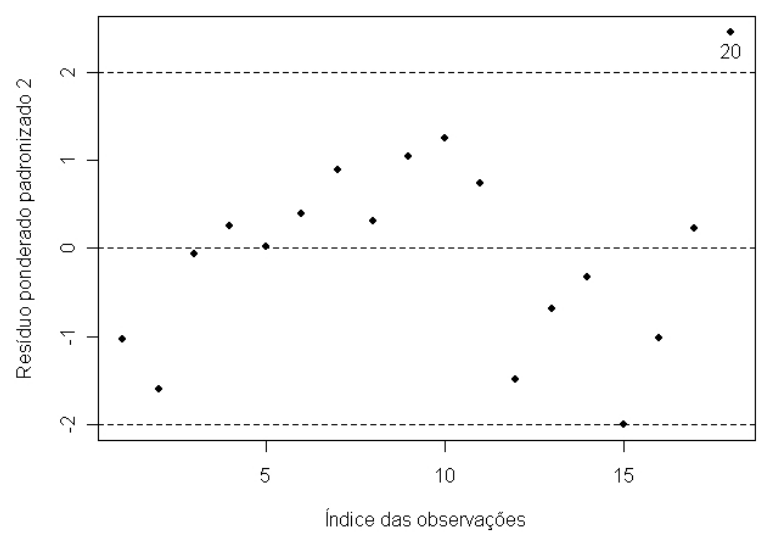

(b)

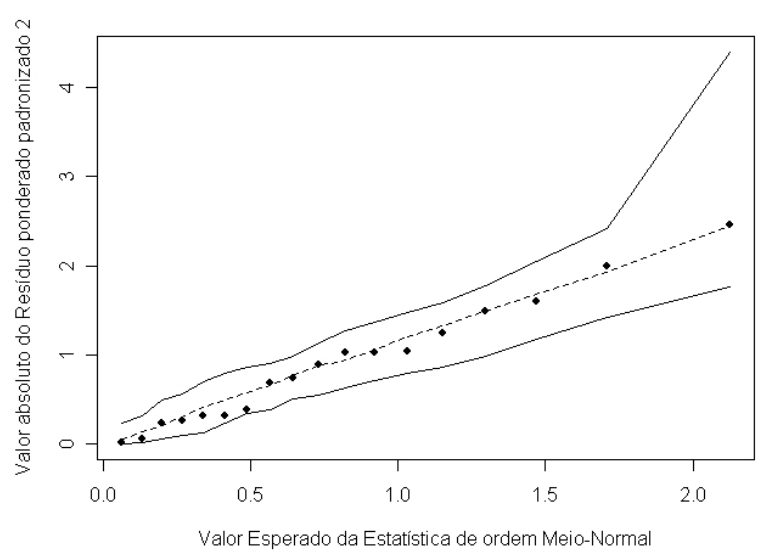

(d)

Figura 5.8: Gráficos de diagnóstico do modelo de regressão simplex ajustado para os dados de oxidação de amônia, sem as observações (4, 17 e 21).

Construímos novamente os gráficos de diagnóstico do modelo ajustado sem as observações (4, 16, 17, 20 e 21) para verificar se há indícios de algum outro ponto alavanca, aberrante e/ou influente (Figura 5.9 a-c). Não aparece nenhum ponto suspeito de alavanca. Apenas a observação 10 se destaca como possível ponto aberrante e influente. Na Figura $5.9 \mathrm{~d}$, os pontos se encontram dentro da banda de confiança.

Ajustando o modelo sem as observações (4, 10, 16, 17, 20 e 21), obtemos as estimativas e variações percentuais dadas na Tabela 5.17. Notamos que a exclusão da observação 10, 


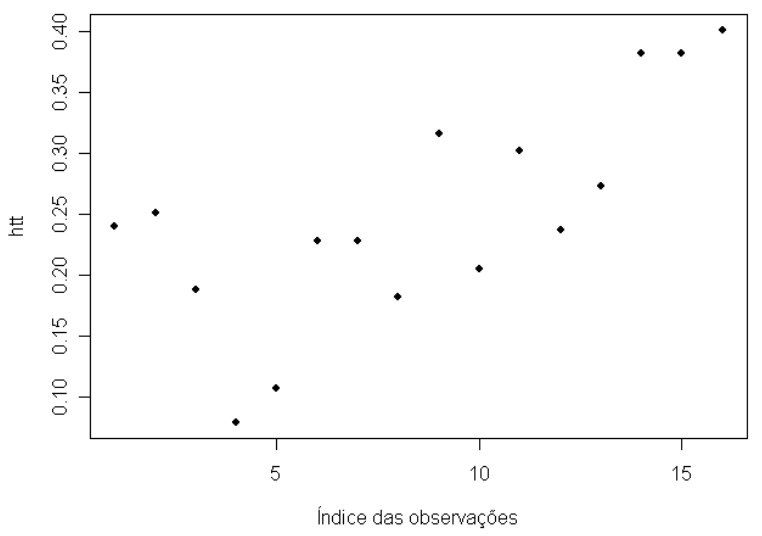

(a)

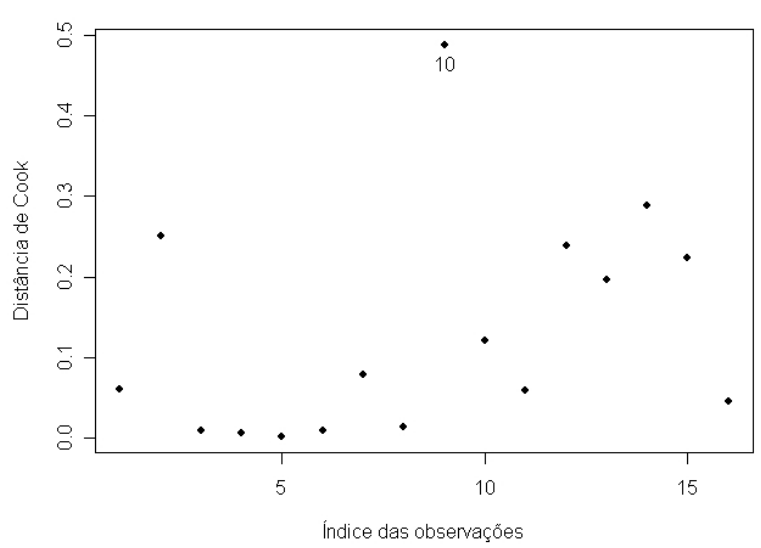

(c)

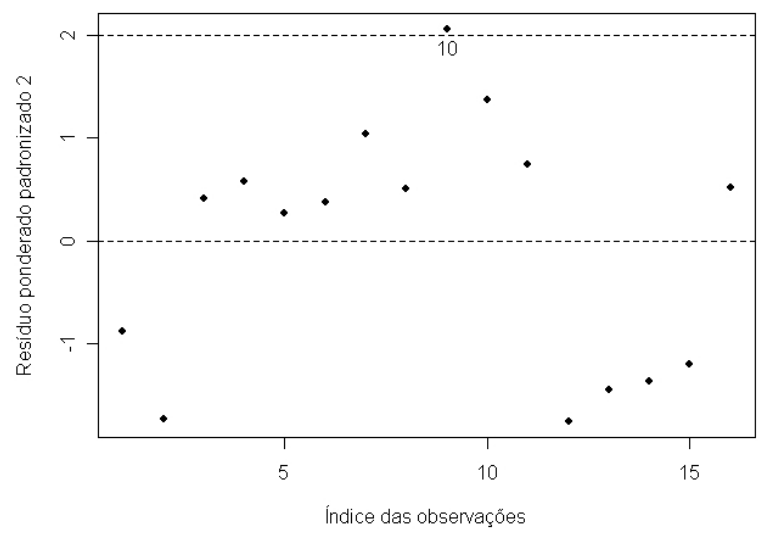

(b)

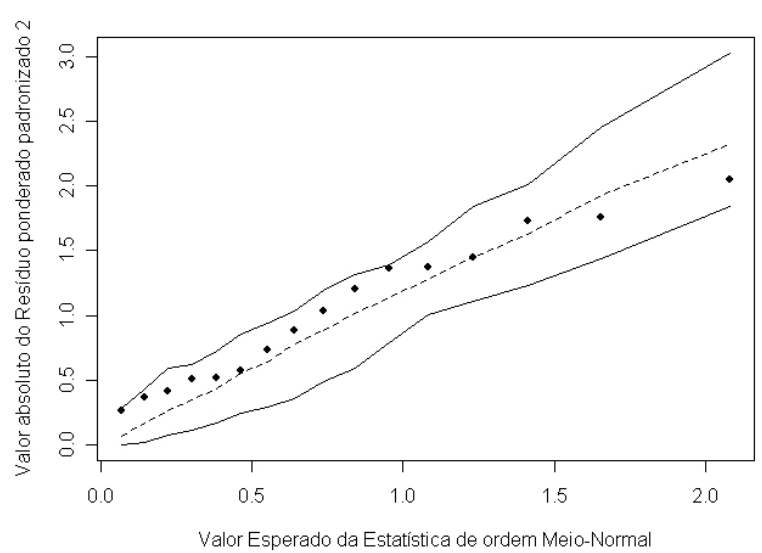

(d)

Figura 5.9: Gráficos de diagnóstico do modelo de regressão simplex ajustado para os dados de oxidação de amônia, sem as observações $(4,16,17,20$ e 21).

retiradas as observações $(4,16,17,20$ e 21), altera as estimativas dos parâmetros.

Fazendo nova análise de diagnóstico, apresentadas na Figura 5.10 , a observação 15 aparece como possível ponto influente. Não se destacam pontos de alavanca ou aberrante. No gráfico de probabilidade meio-normal, notamos que os pontos se encontram dentro da banda de confiança. Por fim, excluindo-se também a observação 15, verificamos que as estimativas se alteram pouco, mas as conclusões inferenciais são as mesmas. 
Tabela 5.17: Variação percentual das estimativas dos parâmetros do modelo de regressão simplex ajustado para os dados de oxidação de amônia, sem as observações (4, 10, 16, 17, 20 e 21).

\begin{tabular}{cccc}
\hline & Sem obs(4, 16, 17, 20 e 21) & \multicolumn{2}{c}{ Sem obs $(4,10,16,17,20$ e 21) } \\
Parâmetro & Estimativa & Estimativa & Var.Percentual \\
\hline$\beta_{0}$ & $-8,0448$ & $-8,4191$ & $4,65 \%$ \\
$\beta_{1}$ & 0,0502 & 0,0462 & $-7,97 \%$ \\
$\beta_{2}$ & 0,0263 & 0,0341 & $29,66 \%$ \\
$\beta_{3}$ & 0,0035 & 0,0085 & $142,86 \%$ \\
$\sigma^{2}$ & 0,4937 & 0,3962 & $-19,75 \%$ \\
\hline
\end{tabular}

Assim, o modelo de regressão simplex se ajusta aos dados de oxidação de amônia, retirando-se as observações $(4,10,16,17,20$ e 21). Em todos os ajustes do modelo de regressão simplex apresentados aqui, a variável concentração de ácido nítrico é não significativa.

Utilizando o critério de informação de Akaike, obtemos $A I C=-174,26$ para o modelo de regressão beta ajustado sem as observações (1, 2, 4 e 21) e $A I C=-149,53$ para o modelo de regressão simplex ajustado sem as observações (4, 10, 16, 17, 20 e 21). O modelo de regressão beta apresenta o menor valor do $A I C$, o que indicaria que seria o modelo mais adequado aos dados. No entanto, deve-se notar que foi necessário excluir muitos pontos para obtenção desses ajustes, sendo que a amostra de dados é muito pequena. 


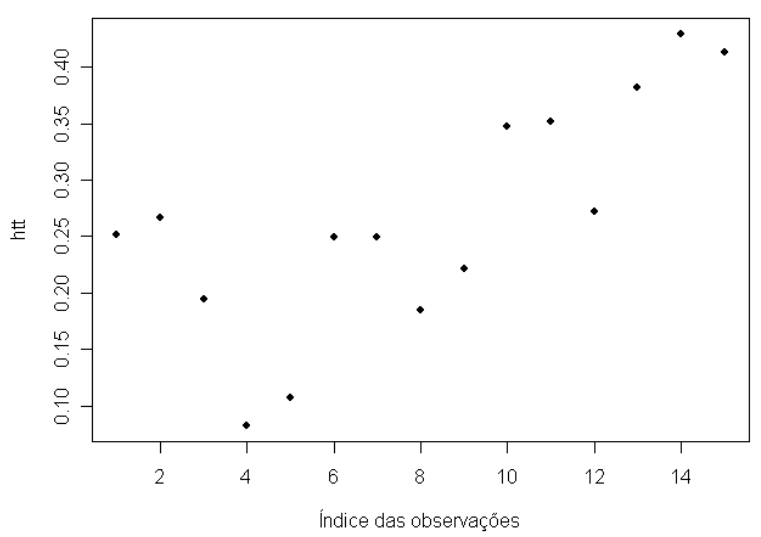

(a)

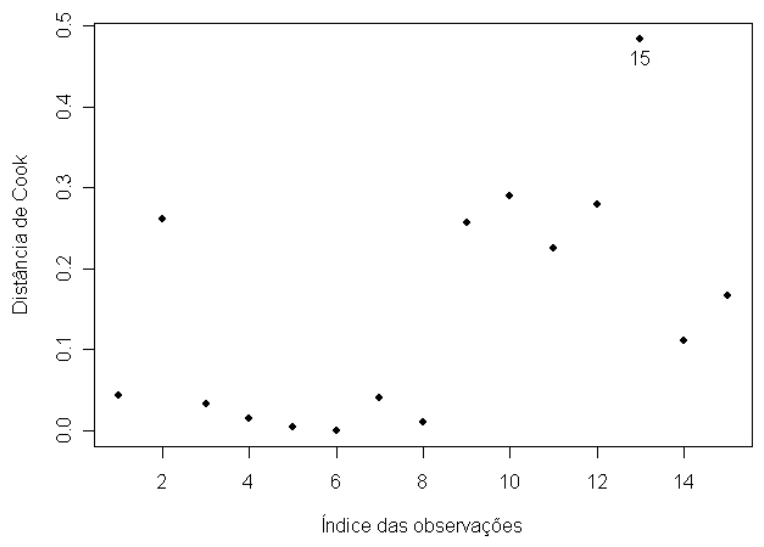

(c)

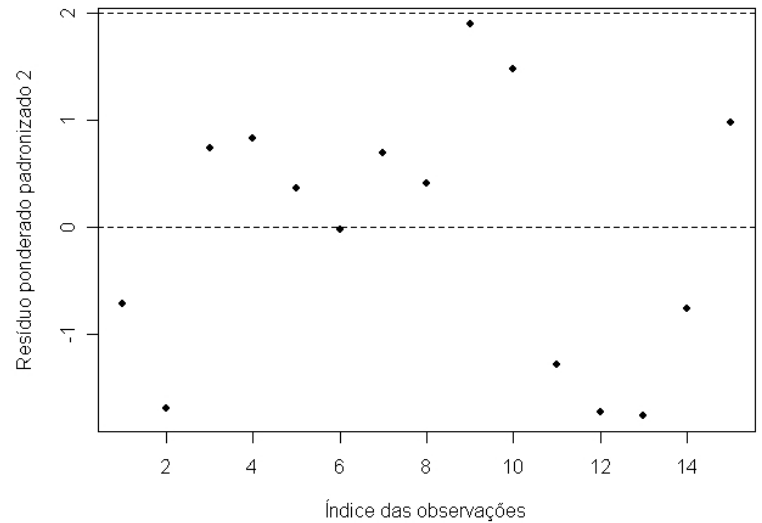

(b)

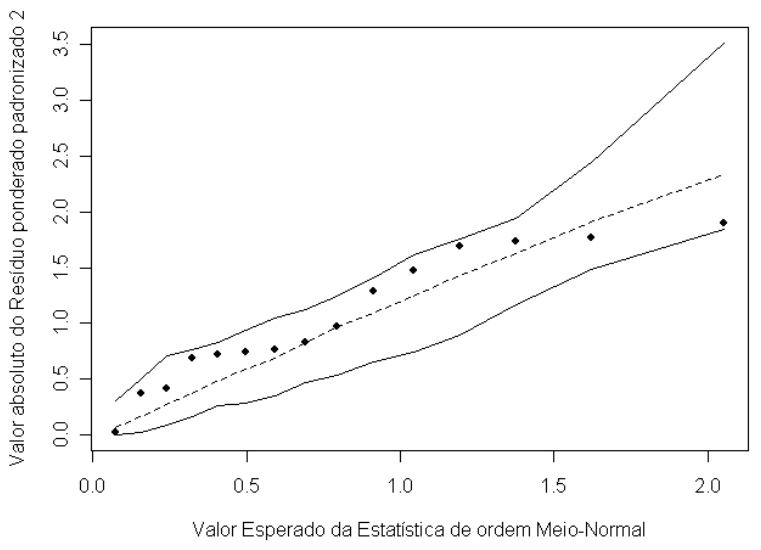

(d)

Figura 5.10: Gráficos de diagnóstico do modelo de regressão simplex ajustado para os dados de oxidação de amônia, sem as observações (4, 10, 16, 17, 20 e 21). 


\section{Capítulo 6}

\section{Conclusões}

Nesta dissertação, apresentamos o modelo de regressão beta proposto por Ferrari \& Cribari-Neto (2004). Algumas funções e características desse modelo também são apresentadas para servirem de base para o desenvolvimento do modelo de regressão baseado na distribuição simplex (Barndorff-Nielsen \& Jørgensen (1991)), como a função escore e a matriz informação de Fisher. Definimos os resíduos ponderados padronizados 2 para o modelo de regressão simplex, seguindo o mesmo princípio de construção dos resíduos propostos por Espinheira et al. (2008). Apresentamos também uma generalização de algumas medidas de diagnóstico, que permitem identificar possíveis pontos de alavanca, aberrantes e/ou influentes a partir da matriz de projeção e do resíduo padronizado. Essas medidas podem ser aplicadas tanto no caso do modelo de regressão beta como no modelo de regressão simplex.

Desenvolvemos uma rotina computacional em $\mathrm{R}$ para ajuste do modelo de regressão simplex, baseada em algumas funções do pacote betareg. Implementamos também um programa para análise de diagnóstico baseado no resíduo ponderado padronizado 2 para os dois modelos. Os gráficos de probabilidade meio-normal com envelope simulado também foram construídos com esse resíduo.

Apresentamos duas aplicações a dados reais para comparar os modelos de regressão beta e simplex. Nessas aplicações, avaliamos os ajustes dos modelos por meio das medidas de diagnóstico. Mostramos, na Aplicação I, que o modelo de regressão beta é o mais adequado para os dados de gasolina. O modelo de regressão simplex também foi ajustado a esses dados, porém, nesse caso, foi necessário excluir alguns pontos. 
Na Aplicação II, o modelo de regressão beta parece ter melhor ajuste que o simplex. No entanto, devido ao pequeno tamanho da amostra e ao número grande de observações excluídas, há uma indicação de que ambos os ajustes não foram satisfatórios. 


\section{Apêndice A}

\section{Função Escore e Matriz de Informação de Fisher na Regressão Beta}

Neste apêndice apresentamos os cálculos para a obtenção da função escore e da matriz de informação de Fisher para os parâmetros $(\beta, \phi)$ da distribuição beta.

O logaritmo natural da função de verossimilhança baseada em uma amostra de $n$ observações independentes é dado por

$$
\ell(\beta, \phi)=\sum_{t=1}^{n} \ell_{t}\left(\mu_{t}, \phi\right),
$$

em que

$$
\begin{aligned}
\ell_{t}\left(\mu_{t}, \phi\right) & =\log \Gamma(\phi)-\log \Gamma\left(\mu_{t} \phi\right)-\log \Gamma\left(\left(1-\mu_{t}\right) \phi\right)+\left(\mu_{t} \phi-1\right) \log y_{t} \\
& +\left\{\left(1-\mu_{t}\right) \phi-1\right\} \log \left(1-y_{t}\right)
\end{aligned}
$$

$\operatorname{com} \mu_{t}=g^{-1}\left(\eta_{t}\right)$ e $g\left(\mu_{t}\right)=\sum_{i=1}^{k} x_{t i} \beta_{i}=\eta_{t}$.

A função escore é obtida derivando-se o logaritmo da função de verossimilhança em relação aos parâmetros desconhecidos. 
Derivando-se $\ell(\beta, \phi)$ em relação a $\beta_{i}$ para $i=1, \ldots, k$, encontramos

$$
\frac{\partial \ell(\beta, \phi)}{\partial \beta_{i}}=\sum_{t=1}^{n} \frac{\partial \ell_{t}\left(\mu_{t}, \phi\right)}{\partial \mu_{t}} \frac{\mathrm{d} \mu_{t}}{\mathrm{~d} \eta_{t}} \frac{\partial \eta_{t}}{\partial \beta_{i}} .
$$

Sendo

$$
\begin{gathered}
\frac{\partial \ell_{t}\left(\mu_{t}, \phi\right)}{\partial \mu_{t}}=\phi\left[\log \frac{y_{t}}{1-y_{t}}-\left\{\psi\left(\mu_{t} \phi\right)-\psi\left(\left(1-\mu_{t}\right) \phi\right)\right\}\right], \\
\frac{\mathrm{d} \mu_{t}}{\mathrm{~d} \eta_{t}}=\frac{1}{g^{\prime}\left(\mu_{t}\right)} \text { e } \frac{\partial \eta_{t}}{\partial \beta_{i}}=x_{t i},
\end{gathered}
$$

a expressão A.2 pode ser escrita como

$$
\frac{\partial \ell(\beta, \phi)}{\partial \beta_{i}}=\phi \sum_{t=1}^{n}\left(y_{t}^{*}-\mu_{t}^{*}\right) \frac{1}{g^{\prime}\left(\mu_{t}\right)} x_{t i},
$$

onde

$$
y_{t}^{*}=\log \left\{\frac{y_{t}}{\left(1-y_{t}\right)}\right\} \text { e } \mu_{t}^{*}=\psi\left(\mu_{t} \phi\right)-\psi\left(\left(1-\mu_{t}\right) \phi\right),
$$

em que $\psi(\cdot)$ é a função digama, definida como

$$
\psi(a)=\frac{\mathrm{d} \log \Gamma(a)}{\mathrm{d} a}=\frac{\Gamma^{\prime}(a)}{\Gamma(a)}, \lambda>0 .
$$

Note que, sob condições de regularidade (ver Sen \& Singer (1993)), o valor esperado de $\partial \ell_{t}\left(\mu_{t}, \phi\right) / \partial \mu_{t}$ é igual a zero. Assim,

$$
\mathrm{E}\left[\frac{\partial \ell_{t}\left(\mu_{t}, \phi\right)}{\partial \mu_{t}}\right]=0 \Leftrightarrow \mathrm{E}\left[\phi\left(y_{t}^{*}-\mu_{t}^{*}\right)\right]=0 \Leftrightarrow \mathrm{E}\left[y_{t}^{*}\right]=\mu_{t}^{*},
$$

o que significa que o valor esperado de $y_{t}^{*}$ é igual a $\mu_{t}^{*}$.

A forma matricial da função escore para $\beta, U_{\beta}(\beta, \phi)$, que é um vetor coluna de dimensão $k$, é

$$
U_{\beta}(\beta, \phi)=\phi X^{\top} T\left(y^{*}-\mu^{*}\right),
$$

em que $X$ é uma matriz $n \times k$, cuja $t$-ésima linha é $x_{t}^{\top}, T=\operatorname{diag}\left\{1 / g^{\prime}\left(\mu_{1}\right), \ldots, 1 / g^{\prime}\left(\mu_{n}\right)\right\}, y^{*}=$ $\left(y_{1}^{*}, \ldots, y_{n}^{*}\right)^{\top}$ e $\mu^{*}=\left(\mu_{1}^{*}, \ldots, \mu_{n}^{*}\right)^{\top}$. 
Analogamente, derivando-se o logaritmo da função de verossimilhança em relação a $\phi$, temos

$$
\frac{\partial(\beta, \phi)}{\partial \phi}=\sum_{t=1}^{n} \frac{\partial \ell_{t}\left(\mu_{t}, \phi\right)}{\partial \phi}
$$

onde

$$
\begin{aligned}
\frac{\partial \ell_{t}\left(\mu_{t}, \phi\right)}{\partial \phi}= & \mu_{t}\left[\log \frac{y_{t}}{1-y_{t}}-\left\{\psi\left(\mu_{t} \phi\right)-\psi\left(\left(1-\mu_{t}\right) \phi\right)\right\}\right]+\log \left(1-y_{t}\right) \\
& -\psi\left(\left(1-\mu_{t}\right) \phi\right)+\psi(\phi) .
\end{aligned}
$$

Logo, a função escore para o parâmetro de precisão $\phi$ é dada por

$$
U_{\phi}(\beta, \phi)=\sum_{t=1}^{n}\left\{\mu_{t}\left(y_{t}^{*}-\mu_{t}^{*}\right)+\log \left(1-y_{t}\right)-\psi\left(\left(1-\mu_{t}\right) \phi\right)+\psi(\phi)\right\},
$$

sendo $U_{\phi}(\beta, \phi)$ um escalar.

O próximo passo é calcular a matriz de informação de Fisher. Da equação A.2 , a segunda derivada de $\ell(\beta, \phi)$ com respeito a $\beta_{i}$ e $\beta_{j}$ é dada por

$$
\begin{aligned}
\frac{\partial^{2} \ell(\beta, \phi)}{\partial \beta_{i} \partial \beta_{j}} & =\sum_{t=1}^{n} \frac{\partial}{\partial \mu_{t}}\left(\frac{\partial \ell_{t}\left(\mu_{t}, \phi\right)}{\partial \mu_{t}} \frac{\mathrm{d} \mu_{t}}{\mathrm{~d} \eta_{t}}\right) \frac{\mathrm{d} \mu_{t}}{\mathrm{~d} \eta_{t}} \frac{\partial \eta_{t}}{\partial \beta_{j}} x_{t i} \\
& =\sum_{t=1}^{n}\left\{\frac{\partial^{2} \ell_{t}\left(\mu_{t}, \phi\right)}{\partial \mu_{t}^{2}} \frac{\mathrm{d} \mu_{t}}{\mathrm{~d} \eta_{t}}+\frac{\partial \ell_{t}\left(\mu_{t}, \phi\right)}{\partial \mu_{t}} \frac{\partial}{\partial \mu_{t}}\left(\frac{\mathrm{d} \mu_{t}}{\mathrm{~d} \eta_{t}}\right)\right\} \frac{\mathrm{d} \mu_{t}}{\mathrm{~d} \eta_{t}} x_{t i} x_{t j} .
\end{aligned}
$$

Como $\mathrm{E}\left(\partial \ell_{t}\left(\mu_{t}, \phi\right) / \partial \mu_{t}\right)=0$, segue que

$$
\mathrm{E}\left(\frac{\partial^{2} \ell(\beta, \phi)}{\partial \beta_{i} \partial \beta_{j}}\right)=\sum_{t=1}^{n} \mathrm{E}\left(\frac{\partial^{2} \ell_{t}\left(\mu_{t}, \phi\right)}{\partial \mu_{t}^{2}}\right)\left(\frac{\mathrm{d} \mu_{t}}{\mathrm{~d} \eta_{t}}\right)^{2} x_{t i} x_{t j},
$$

Agora, da equação A.3 temos

$$
\frac{\partial^{2} \ell_{t}\left(\mu_{t}, \phi\right)}{\partial \mu_{t}^{2}}=-\phi^{2}\left\{\psi^{\prime}\left(\mu_{t} \phi\right)+\psi^{\prime}\left(\left(1-\mu_{t}\right) \phi\right)\right\},
$$


em que $\psi^{\prime}(\cdot)$ é a função trigama definida como

$$
\psi^{\prime}(a)=\frac{\mathrm{d} \psi(a)}{\mathrm{d} a}=\frac{\mathrm{d}^{2} \log \Gamma(a)}{\mathrm{d} a^{2}}=\frac{\Gamma^{\prime \prime}(a) \Gamma(a)-\left[\Gamma^{\prime}(a)\right]^{2}}{[\Gamma(a)]^{2}}, \quad a>0 .
$$

Assim

$$
\mathrm{E}\left(\frac{\partial^{2} \ell(\beta, \phi)}{\partial \beta_{i} \partial \beta_{j}}\right)=-\phi \sum_{t=1}^{n} w_{t} x_{t i} x_{t j}, \quad \operatorname{com} w_{t}=\phi\left\{\psi^{\prime}\left(\mu_{t} \phi\right)+\psi^{\prime}\left(\left(1-\mu_{t}\right) \phi\right)\right\} \frac{1}{\left\{g^{\prime}\left(\mu_{t}\right)\right\}^{2}} .
$$

Em notação matricial,

$$
\mathrm{E}\left(\frac{\partial^{2} \ell(\beta, \phi)}{\partial \beta \partial \beta^{\top}}\right)=-\phi X^{\top} W X, \operatorname{com} W=\operatorname{diag}\left\{w_{1}, \ldots, w_{n}\right\}
$$

De (A.4), a derivada de segunda ordem de $\ell(\beta, \phi)$ em relação a $\beta_{i}$ e $\phi$ pode ser escrita como

$$
\frac{\partial^{2} \ell(\beta, \phi)}{\partial \beta_{i} \partial \phi}=\sum_{t=1}^{n}\left[\left(y_{t}^{*}-\mu_{t}^{*}\right)-\phi \frac{\partial \mu_{t}^{*}}{\partial \phi}\right] \frac{1}{g^{\prime}\left(\mu_{t}\right)} x_{t i} .
$$

Como

$$
\mathrm{E}\left(y_{t}^{*}\right)=\mu_{t}^{*} \quad \text { e } \frac{\partial \mu_{t}^{*}}{\partial \phi}=\psi^{\prime}\left(\mu_{t} \phi\right) \mu_{t}-\psi^{\prime}\left(\left(1-\mu_{t}\right) \phi\right)\left(1-\mu_{t}\right),
$$

segue que

$$
\mathrm{E}\left(\frac{\partial^{2} \ell(\beta, \phi)}{\partial \beta_{i} \partial \phi}\right)=-\sum_{t=1}^{n} c_{t} \frac{1}{g^{\prime}\left(\mu_{t}\right)} x_{t i}, \quad \operatorname{com} c_{t}=\phi\left\{\psi^{\prime}\left(\mu_{t} \phi\right) \mu_{t}-\psi^{\prime}\left(\left(1-\mu_{t}\right) \phi\right)\left(1-\mu_{t}\right)\right\} .
$$

A forma matricial é dada por,

$$
\mathrm{E}\left(\frac{\partial^{2} \ell(\beta, \phi)}{\partial \beta \partial \phi}\right)=-X^{\top} T c, \quad \operatorname{com} c=\left(c_{1}, \ldots, c_{n}\right)^{\top} \text { e } T=\operatorname{diag}\left\{1 / g^{\prime}\left(\mu_{1}\right), \ldots, 1 / g^{\prime}\left(\mu_{n}\right)\right\} .
$$

Por último, diferenciando a expressão A.6 em relação a $\phi$,

$$
\frac{\partial^{2} \ell(\beta, \phi)}{\partial \phi^{2}}=\sum_{t=1}^{n} \frac{\partial^{2} \ell\left(\mu_{t}, \phi\right)}{\partial \phi^{2}}=-\sum_{t=1}^{n}\left[\psi^{\prime}\left(\mu_{t} \phi\right) \mu_{t}^{2}+\psi^{\prime}\left(\left(1-\mu_{t}\right) \phi\right)\left(1-\mu_{t}\right)^{2}-\psi^{\prime}(\phi)\right] .
$$


Definindo $D=\operatorname{diag}\left(d_{1}, \ldots, d_{n}\right)$, com $d_{t}=\psi^{\prime}\left(\mu_{t} \phi\right) \mu_{t}^{2}+\psi^{\prime}\left(\left(1-\mu_{t}\right) \phi\right)\left(1-\mu_{t}\right)^{2}-\psi^{\prime}(\phi)$, obtemos

$$
\mathrm{E}\left(\frac{\partial^{2} \ell(\beta, \phi)}{\partial \phi^{2}}\right)=-\sum_{t=1}^{n} d_{t} .
$$

Matricialmente, podemos escrever (A.9) como

$$
\mathrm{E}\left(\frac{\partial^{2} \ell(\beta, \phi)}{\partial \phi^{2}}\right)=-\operatorname{tr}(D),
$$

em que $\operatorname{tr}(D)$ é o traço da matriz $D$.

Finalmente, a matriz de informação de Fisher para $(\beta, \phi)$ é dada por

$$
K=K(\beta, \phi)=\left(\begin{array}{cc}
K_{\beta \beta} & K_{\beta \phi} \\
K_{\phi \beta} & K_{\phi \phi}
\end{array}\right),
$$

onde $K_{\beta \beta}=\phi X^{\top} W X, K_{\beta \phi}=K_{\phi \beta}=X^{\top} T c$ e $K_{\phi \phi}=\operatorname{tr}(D)$. Note que os parâmetros $\beta$ e $\phi$ não são ortogonais, ao contrário do que acontece na classe de modelos lineares generalizados (McCullagh \& Nelder (1989)). 


\section{Apêndice B}

\section{Propriedades da Distribuição Simplex}

Neste apêndice apresentamos algumas propriedades da distribuição simplex (ver Song \& $\operatorname{Tan}(2000)$ ).

Consideremos $y \sim S^{-}\left(\mu, \sigma^{2}\right), 0<\mu<1$ e $\sigma^{2}>0$, cuja função densidade é dada por

$$
p\left(y ; \mu, \sigma^{2}\right)=\left[2 \pi \sigma^{2}\{y(1-y)\}^{3}\right]^{-1 / 2} \exp \left\{-\frac{1}{2 \sigma^{2}} d(y ; \mu)\right\}, y \in(0,1),
$$

onde

$$
d(y ; \mu)=\frac{(y-\mu)^{2}}{y(1-y) \mu^{2}(1-\mu)^{2}} .
$$

\section{Propriedades:}

(1) Definindo $u=-(1 / 2) d^{\prime}(y ; \mu)$, com $d^{\prime}(y ; \mu)=\partial d(y ; \mu) / \partial \mu$, temos

$$
\begin{aligned}
u & =-\frac{1}{2} d^{\prime}(y ; \mu)=-\frac{1}{2} \frac{\partial d(y ; \mu)}{\partial \mu}=\frac{(y-\mu)\left(y-2 \mu y+\mu^{2}\right)}{y(1-y) \mu^{3}(1-\mu)^{3}} \\
& =\frac{(y-\mu)}{\mu(1-\mu)}\left\{d(y ; \mu)+\frac{1}{\mu^{2}(1-\mu)^{2}}\right\} .
\end{aligned}
$$


(2) $\mathrm{E}\left[(y-\mu) d^{\prime}(y ; \mu)\right]=-2 \sigma^{2}$.

Temos que

$$
\mathrm{E}(y-\mu)=0=\int_{0}^{1}(y-\mu) p\left(y ; \mu, \sigma^{2}\right) d y .
$$

Diferenciando ambos os lados em relação a $\mu$, obtém-se

$$
0=-1-\frac{1}{2 \sigma^{2}} \mathrm{E}\left[(y-\mu) d^{\prime}(y ; \mu)\right]
$$

e assim,

$$
\mathrm{E}\left[(y-\mu) d^{\prime}(y ; \mu)\right]=-2 \sigma^{2} .
$$

(3) $\mathrm{E}[d(y ; \mu)]=\sigma^{2}$ e $\mathrm{E}[(y-\mu) d(y ; \mu)]=0$.

(4) $\frac{1}{2} \mathrm{E}\left[d^{\prime \prime}(y ; \mu)\right]=\frac{3 \sigma^{2}}{\mu(1-\mu)}+\frac{1}{\mu^{3}(1-\mu)^{3}}, \quad \operatorname{com} d^{\prime \prime}(y ; \mu)=\frac{\partial^{2} d(y ; \mu)}{\partial \mu^{2}}$.

Diferenciando $d^{\prime}(y ; \mu)$ com respeito a $\mu$, obtemos

$$
\begin{aligned}
\frac{1}{2} d^{\prime \prime}(y ; \mu)= & \frac{1}{\mu(1-\mu)}+\frac{(1-2 \mu)}{\mu^{2}(1-\mu)^{2}}(y-\mu) d(y ; \mu) \\
& +\frac{1}{\mu^{3}(1-\mu)^{3}}+\frac{1-2 \mu}{\mu^{4}(1-\mu)^{4}}(y-\mu) \\
& -\frac{1}{\mu(1-\mu)}(y-\mu) d^{\prime}(y ; \mu)-\frac{2(2 \mu-1)}{\mu^{4}(1-\mu)^{4}}(y-\mu) .
\end{aligned}
$$

Então,

$$
\begin{aligned}
\frac{1}{2} \mathrm{E}\left[d^{\prime \prime}(y ; \mu)\right]= & \frac{1}{\mu(1-\mu)}\left\{\mathrm{E} d(y ; \mu)-\mathrm{E}(y-\mu) d^{\prime}(y ; \mu)\right\} \\
& +\frac{1-2 \mu}{\mu^{2}(1-\mu)^{2}} \mathrm{E}(y-\mu) d(y ; \mu)+\frac{1}{\mu^{3}(1-\mu)^{3}} \\
= & \frac{3 \sigma^{2}}{\mu(1-\mu)}+\frac{1}{\mu^{3}(1-\mu)^{3}} .
\end{aligned}
$$


(5) $\mathrm{E}(u)=0$.

Utilizando as propriedades (B.1) e (B.3), segue que

$$
\begin{aligned}
\mathrm{E}(u) & =\mathrm{E}\left[\frac{(y-\mu)}{\mu(1-\mu)}\left\{d(y ; \mu)+\frac{1}{\mu^{2}(1-\mu)^{2}}\right\}\right] \\
& =\frac{1}{\mu(1-\mu)}\left\{\mathrm{E}[(y-\mu) d(y ; \mu)]+\frac{1}{\mu^{2}(1-\mu)^{2}} \mathrm{E}(y-\mu)\right\}=0 .
\end{aligned}
$$




\section{Apêndice $\mathrm{C}$}

\section{Conjunto de Dados}

Neste apêndice, apresentamos os conjuntos de dados referentes às aplicações do Capítulo 5. 
Tabela C.1: Dados de gasolina referentes à Aplicação I.

\begin{tabular}{cccccccccccc}
\hline \multicolumn{1}{c}{ Proporção de } & \multicolumn{1}{c}{ Tipo de petróleo } \\
\hline 1 & petróleo & 1 & 2 & 3 & 4 & 5 & 6 & 7 & 8 & 9 & Temperatura \\
2 & 0,122 & 1 & 0 & 0 & 0 & 0 & 0 & 0 & 0 & 0 & 205 \\
3 & 0,223 & 1 & 0 & 0 & 0 & 0 & 0 & 0 & 0 & 0 & 275 \\
4 & 0,347 & 1 & 0 & 0 & 0 & 0 & 0 & 0 & 0 & 0 & 345 \\
5 & 0,457 & 1 & 0 & 0 & 0 & 0 & 0 & 0 & 0 & 0 & 407 \\
6 & 0,080 & 0 & 1 & 0 & 0 & 0 & 0 & 0 & 0 & 0 & 218 \\
7 & 0,131 & 0 & 1 & 0 & 0 & 0 & 0 & 0 & 0 & 0 & 273 \\
8 & 0,266 & 0 & 1 & 0 & 0 & 0 & 0 & 0 & 0 & 0 & 347 \\
9 & 0,074 & 0 & 0 & 1 & 0 & 0 & 0 & 0 & 0 & 0 & 212 \\
10 & 0,182 & 0 & 0 & 1 & 0 & 0 & 0 & 0 & 0 & 0 & 272 \\
11 & 0,304 & 0 & 0 & 1 & 0 & 0 & 0 & 0 & 0 & 0 & 340 \\
12 & 0,069 & 0 & 0 & 0 & 1 & 0 & 0 & 0 & 0 & 0 & 235 \\
13 & 0,152 & 0 & 0 & 0 & 1 & 0 & 0 & 0 & 0 & 0 & 300 \\
14 & 0,336 & 0 & 0 & 0 & 1 & 0 & 0 & 0 & 0 & 0 & 365 \\
15 & 0,144 & 0 & 0 & 0 & 1 & 0 & 0 & 0 & 0 & 0 & 410 \\
16 & 0,268 & 0 & 0 & 0 & 0 & 1 & 0 & 0 & 0 & 0 & 367 \\
17 & 0,349 & 0 & 0 & 0 & 0 & 1 & 0 & 0 & 0 & 0 & 395 \\
18 & 0,100 & 0 & 0 & 0 & 0 & 0 & 1 & 0 & 0 & 0 & 267 \\
19 & 0,248 & 0 & 0 & 0 & 0 & 0 & 1 & 0 & 0 & 0 & 360 \\
20 & 0,317 & 0 & 0 & 0 & 0 & 0 & 1 & 0 & 0 & 0 & 402 \\
21 & 0,028 & 0 & 0 & 0 & 0 & 0 & 0 & 1 & 0 & 0 & 235 \\
22 & 0,064 & 0 & 0 & 0 & 0 & 0 & 0 & 1 & 0 & 0 & 275 \\
23 & 0,161 & 0 & 0 & 0 & 0 & 0 & 0 & 1 & 0 & 0 & 358 \\
24 & 0,278 & 0 & 0 & 0 & 0 & 0 & 0 & 1 & 0 & 0 & 416 \\
25 & 0,050 & 0 & 0 & 0 & 0 & 0 & 0 & 0 & 1 & 0 & 285 \\
26 & 0,176 & 0 & 0 & 0 & 0 & 0 & 0 & 0 & 1 & 0 & 365 \\
27 & 0,321 & 0 & 0 & 0 & 0 & 0 & 0 & 0 & 1 & 0 & 444 \\
28 & 0,140 & 0 & 0 & 0 & 0 & 0 & 0 & 0 & 0 & 1 & 351 \\
29 & 0,232 & 0 & 0 & 0 & 0 & 0 & 0 & 0 & 0 & 1 & 424 \\
30 & 0,085 & 0 & 0 & 0 & 0 & 0 & 0 & 0 & 0 & 0 & 365 \\
31 & 0,147 & 0 & 0 & 0 & 0 & 0 & 0 & 0 & 0 & 0 & 379 \\
32 & 0,180 & 0 & 0 & 0 & 0 & 0 & 0 & 0 & 0 & 0 & 428 \\
\hline & & & & & & & & & &
\end{tabular}


Tabela C.2: Dados de oxidação de amônia referentes à Aplicação II.

\begin{tabular}{ccccc}
\hline Obs & $\begin{array}{c}\text { Perda na conversão } \\
\text { de amônia }\end{array}$ & $\begin{array}{c}\text { Corrente } \\
\text { de ar }\end{array}$ & $\begin{array}{c}\text { Temperatura } \\
\text { da água }\end{array}$ & $\begin{array}{c}\text { Concentração } \\
\text { de ácido }\end{array}$ \\
\hline 1 & 0,042 & 80 & 27 & 89 \\
2 & 0,037 & 80 & 27 & 88 \\
3 & 0,037 & 75 & 25 & 90 \\
4 & 0,028 & 62 & 24 & 87 \\
5 & 0,018 & 62 & 22 & 87 \\
6 & 0,018 & 62 & 23 & 87 \\
7 & 0,019 & 62 & 24 & 93 \\
8 & 0,02 & 62 & 24 & 93 \\
9 & 0,015 & 58 & 23 & 87 \\
10 & 0,014 & 58 & 18 & 80 \\
11 & 0,014 & 58 & 18 & 89 \\
12 & 0,013 & 58 & 17 & 88 \\
13 & 0,011 & 58 & 18 & 82 \\
14 & 0,012 & 58 & 19 & 93 \\
15 & 0,008 & 50 & 18 & 89 \\
16 & 0,007 & 50 & 18 & 86 \\
17 & 0,008 & 50 & 19 & 72 \\
18 & 0,008 & 50 & 19 & 79 \\
19 & 0,009 & 50 & 20 & 80 \\
20 & 0,015 & 56 & 20 & 82 \\
21 & 0,015 & 70 & 20 & 91 \\
\hline
\end{tabular}




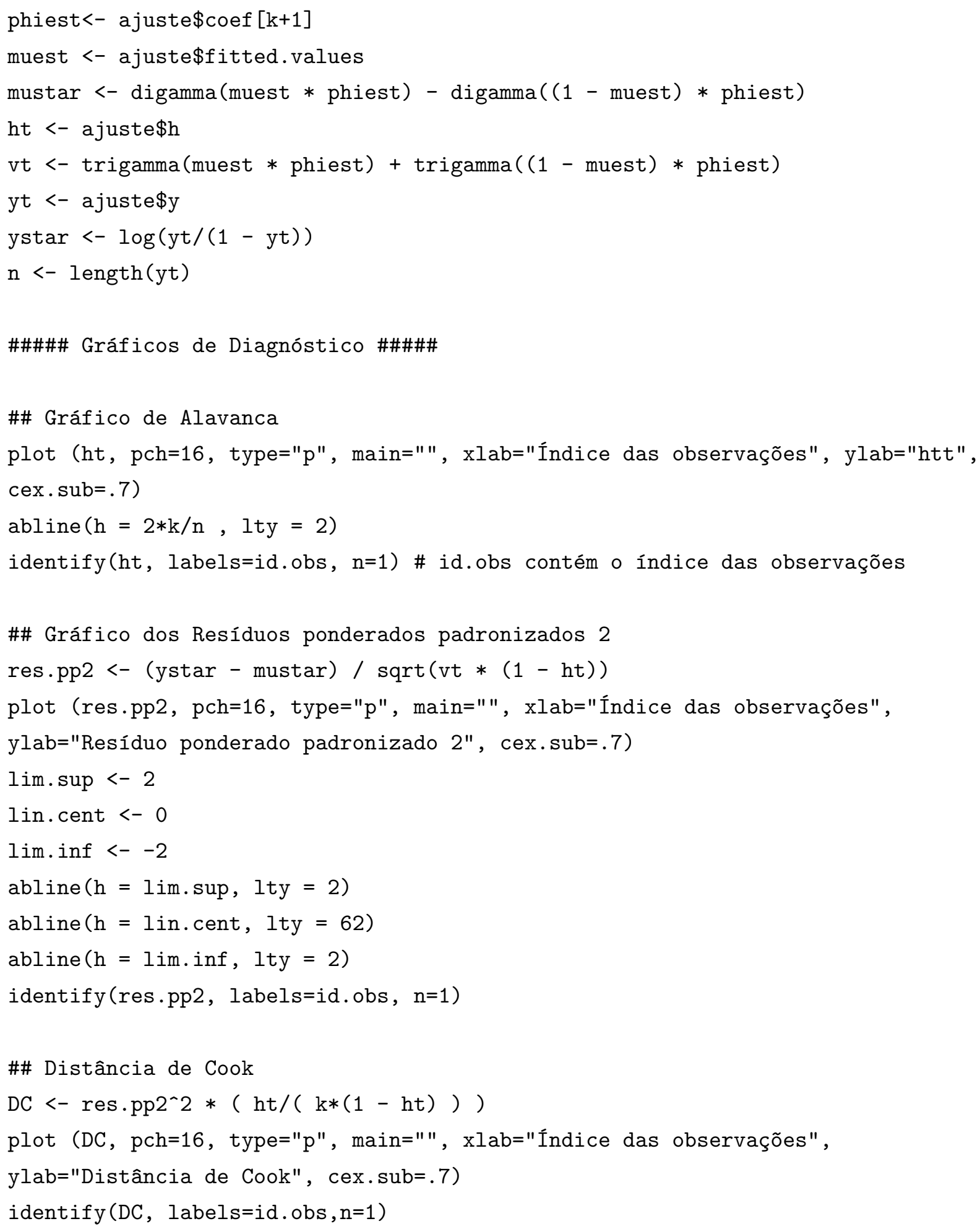


\#\# Gráfico de probabilidade meio-normal com envelope simulado

source ("C: \\envelbeta.rpp2.txt")

\#o arquivo "envelbeta.rpp2.txt" contém a função "envelbeta.rpp2"

envelbeta.rpp2(ajuste, res.pp2)

\#\# AIC

lveros <- sum(lgamma(phiest) - lgamma(muest*phiest) - lgamma((1-muest)*phiest)

$+($ muest*phiest -1$) * \log (y t)+((1-$ muest $) *$ phiest -1$) * \log (1-y t))$

AIC. beta $<-\quad-2 *$ lveros $+2 * \mathrm{k}$

AIC. beta

\section{Programa para a construção do gráfico de probabilidade meio- normal com envelope simulado para o modelo de regressão beta}

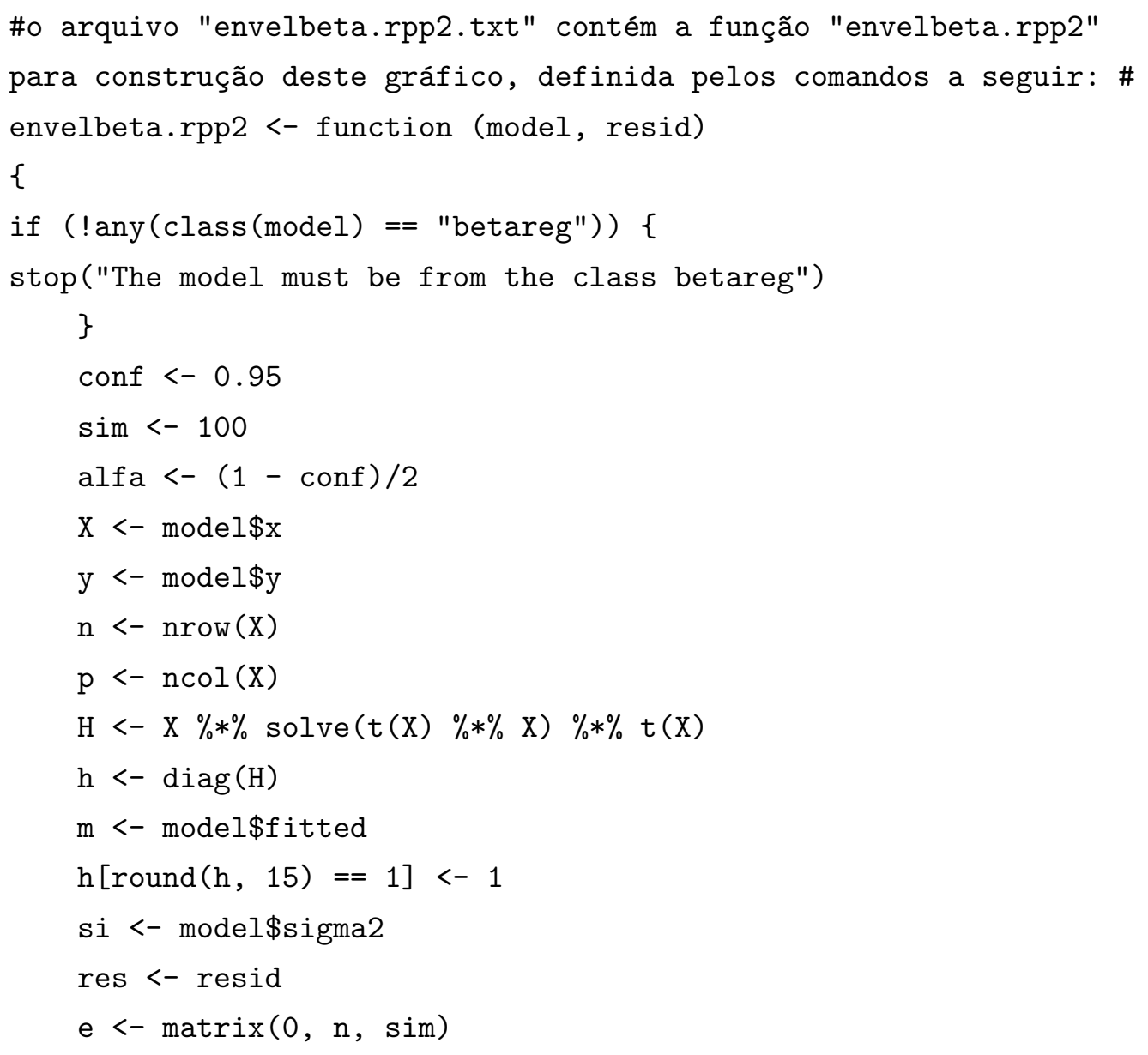




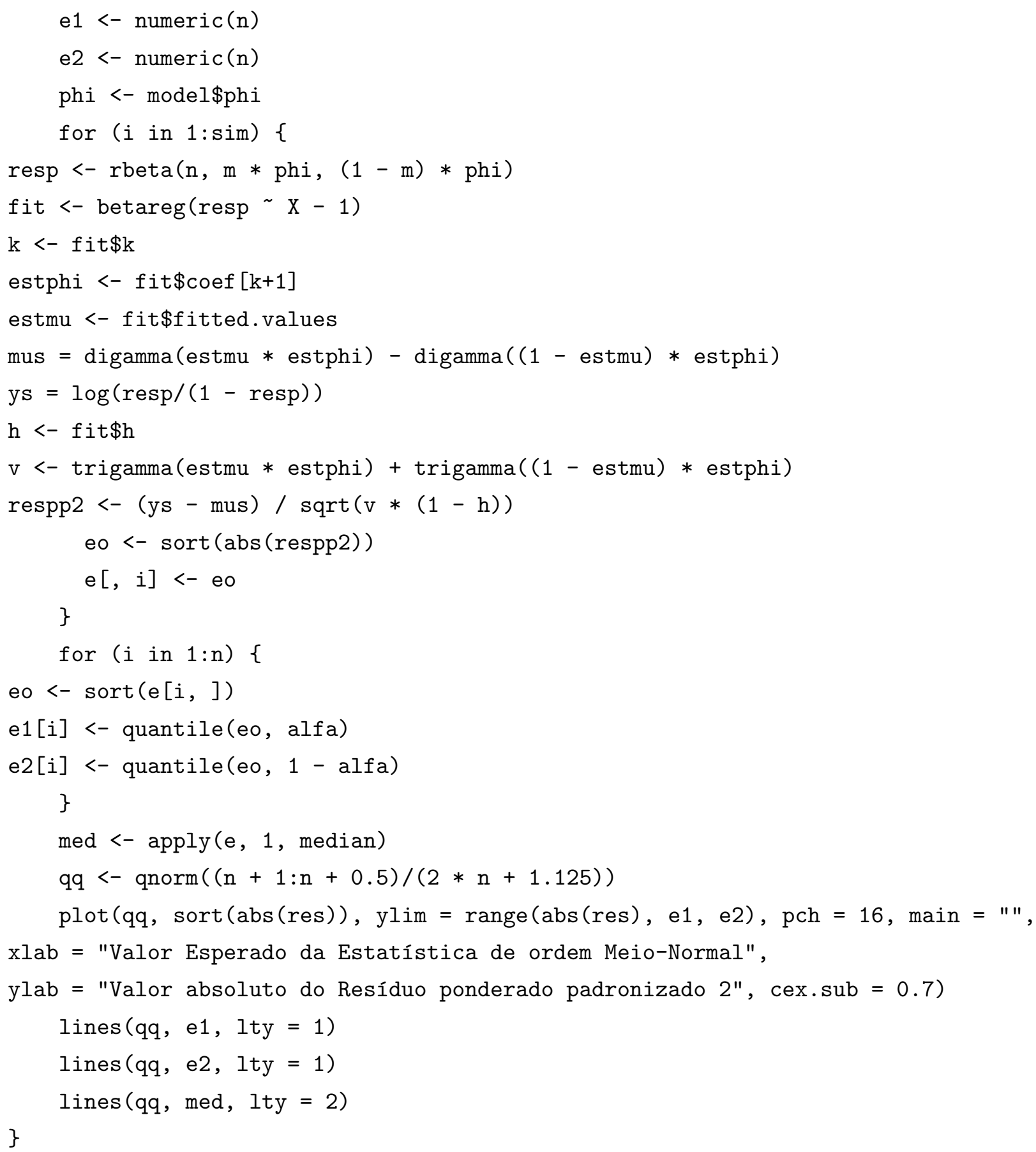




\section{D.2 Programas para ajuste do Modelo de Regressão Simplex}

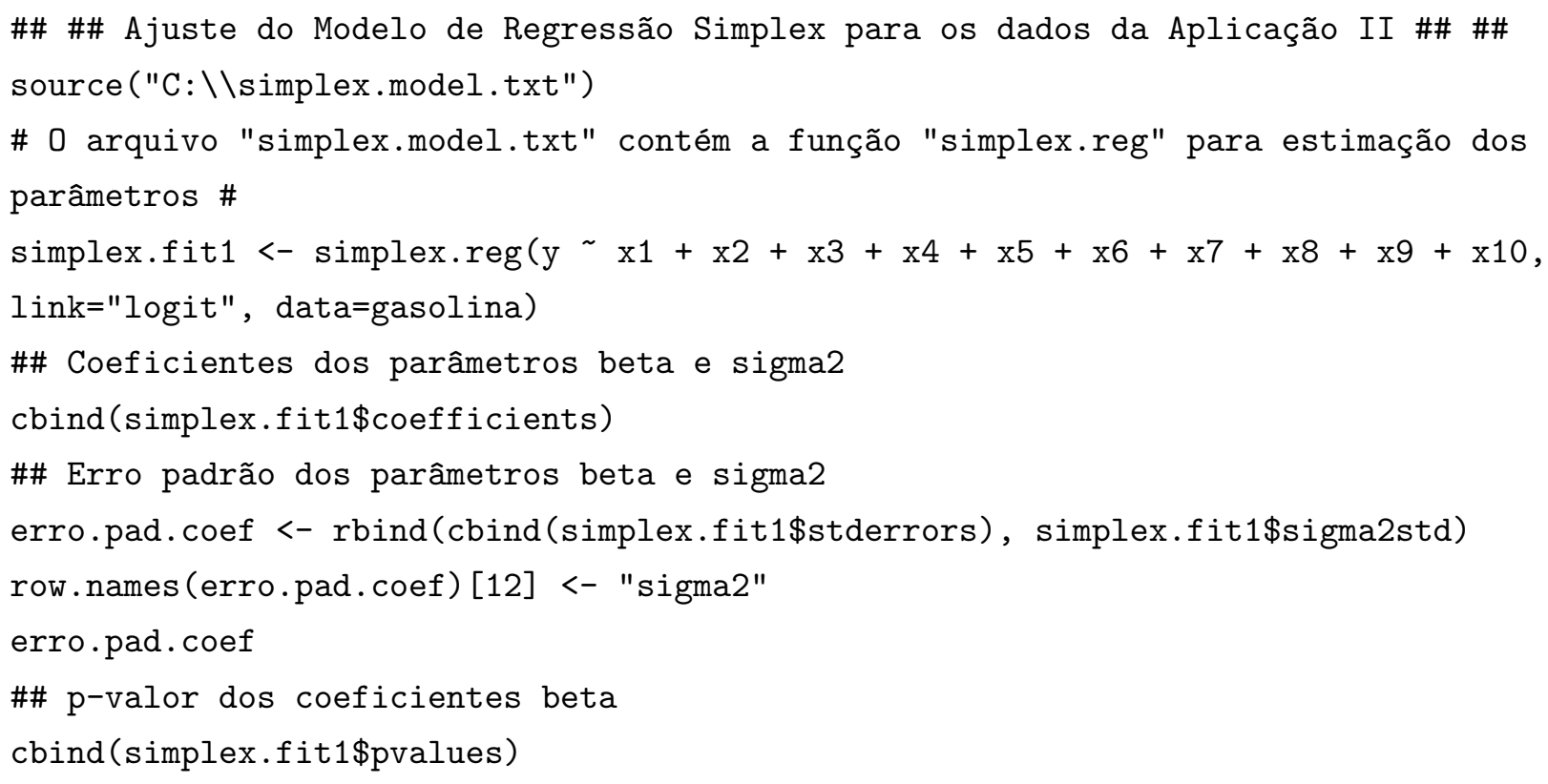

\section{Programa para estimação dos parâmetros do modelo de regressão simplex}

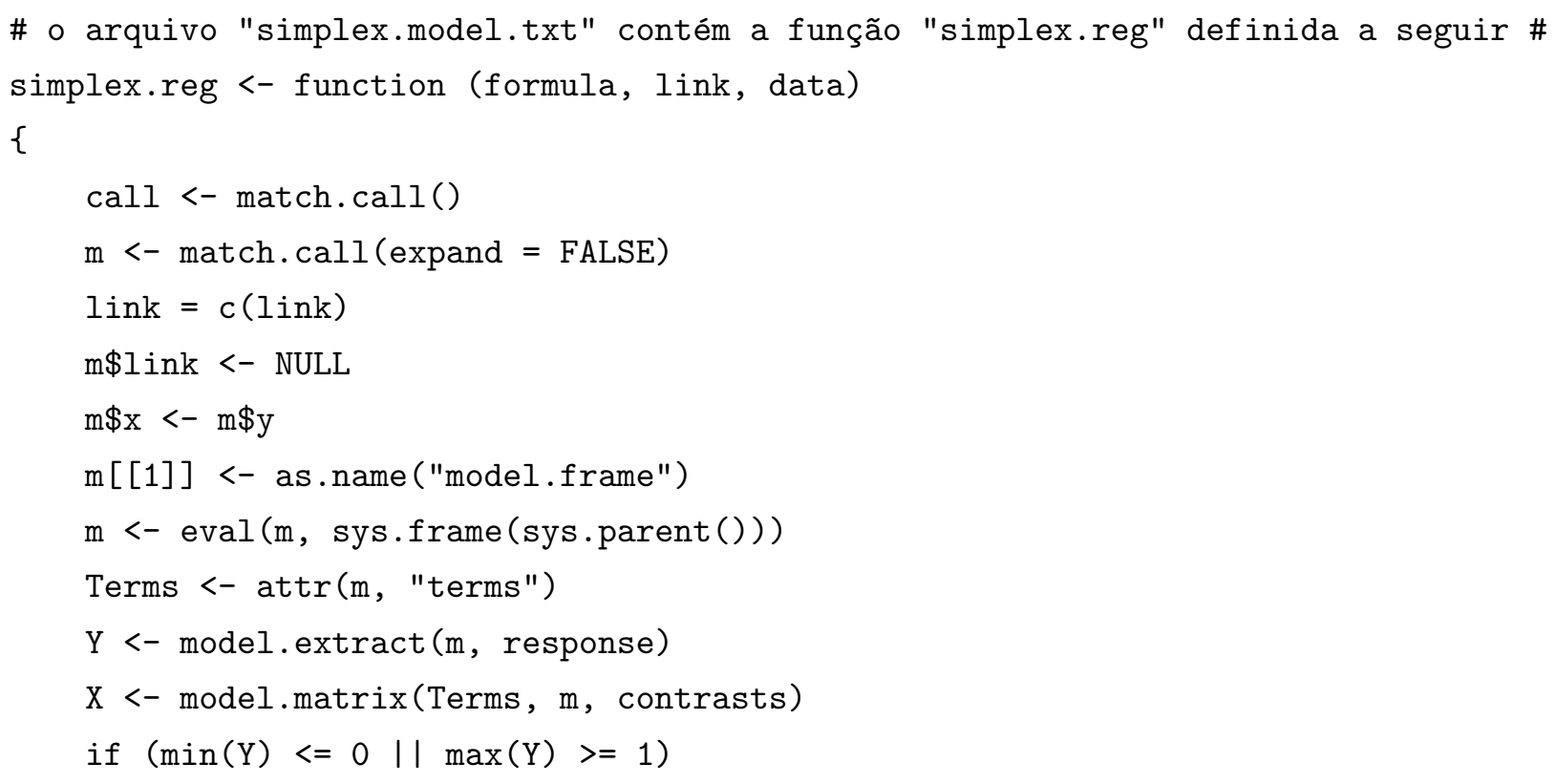




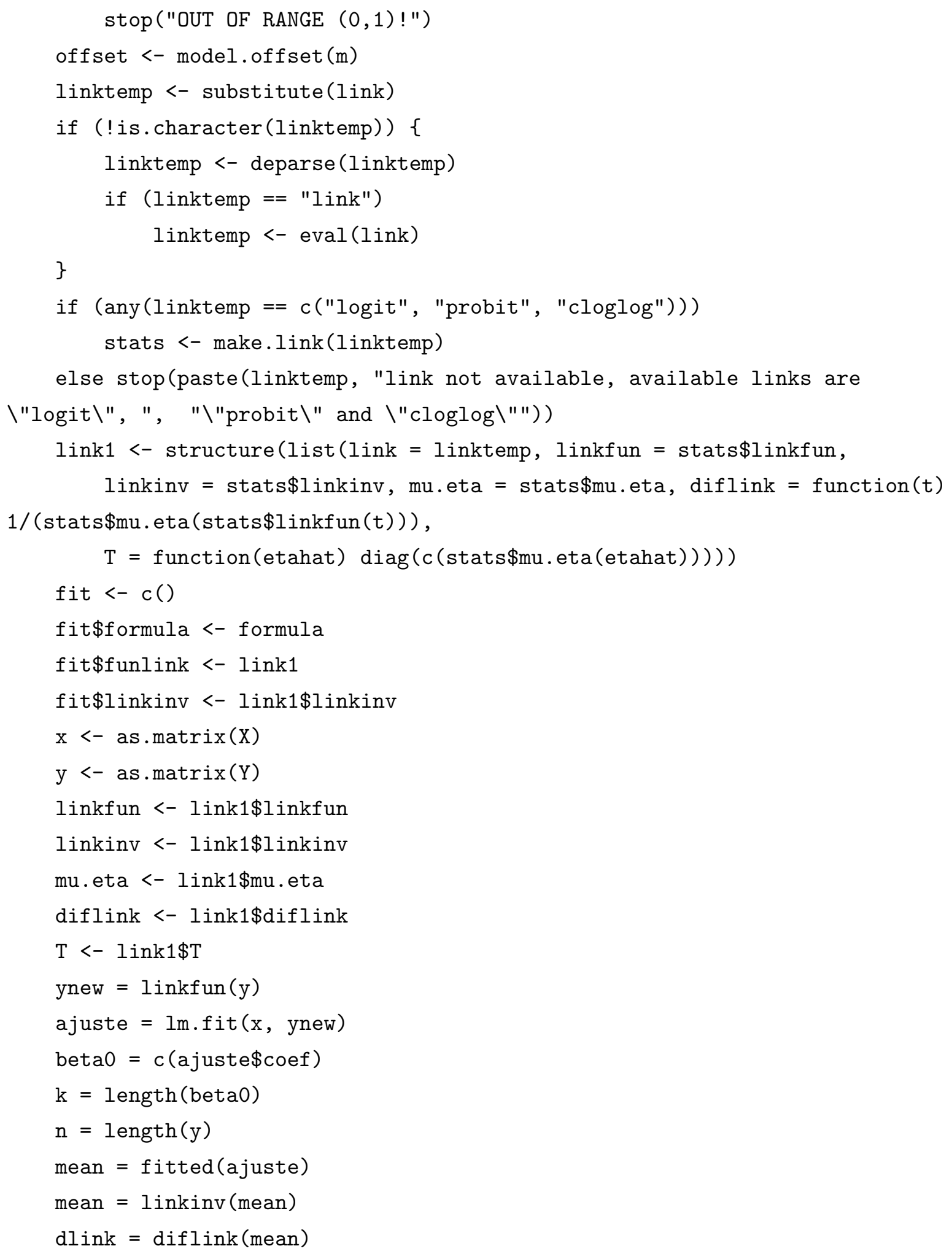




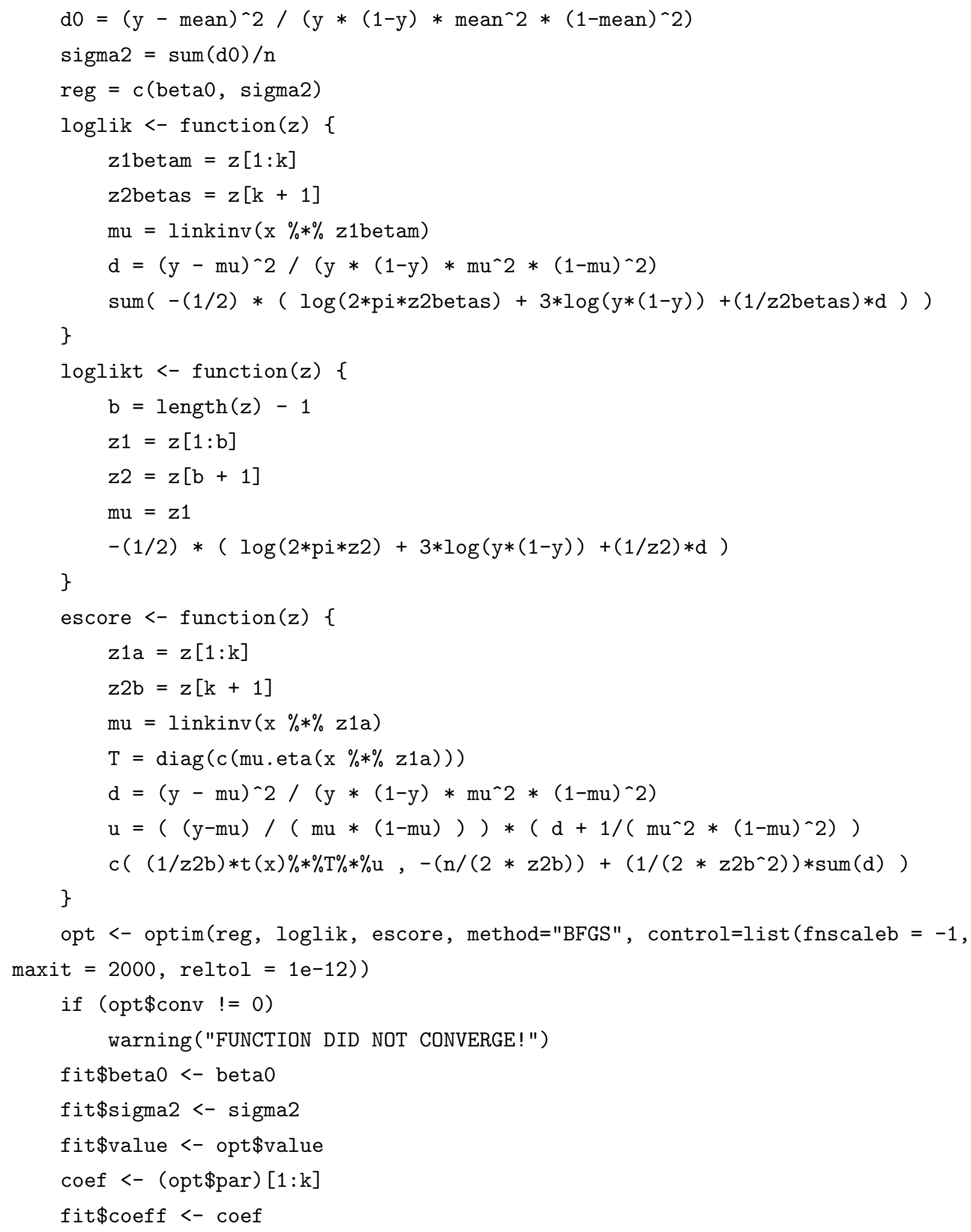




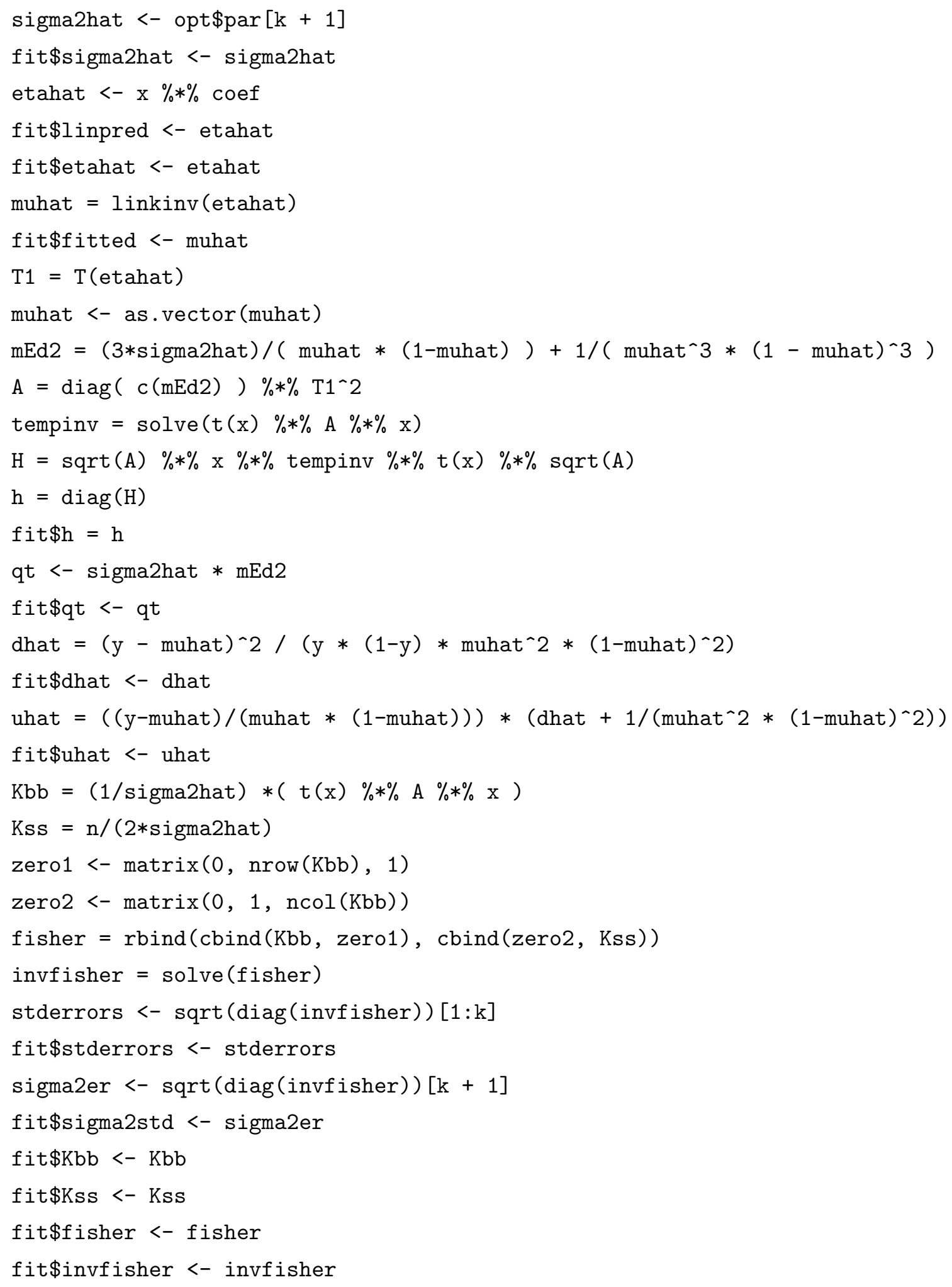




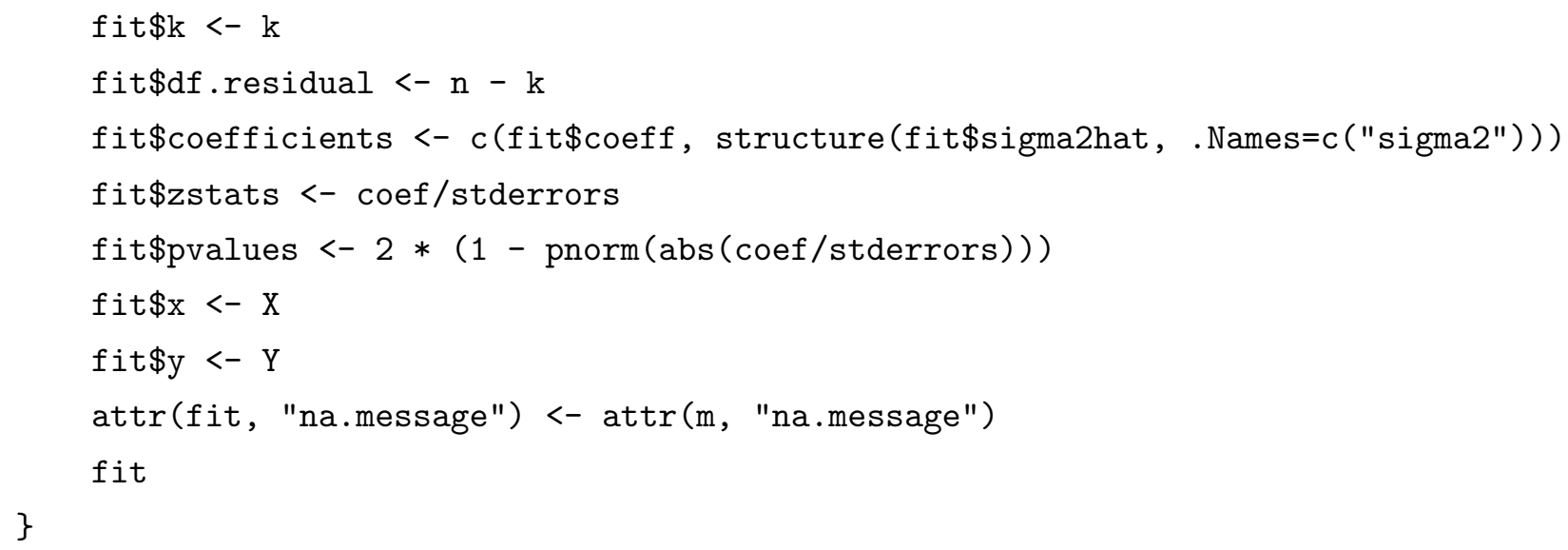

Programa para análise de diagnóstico para o modelo de regressão simplex

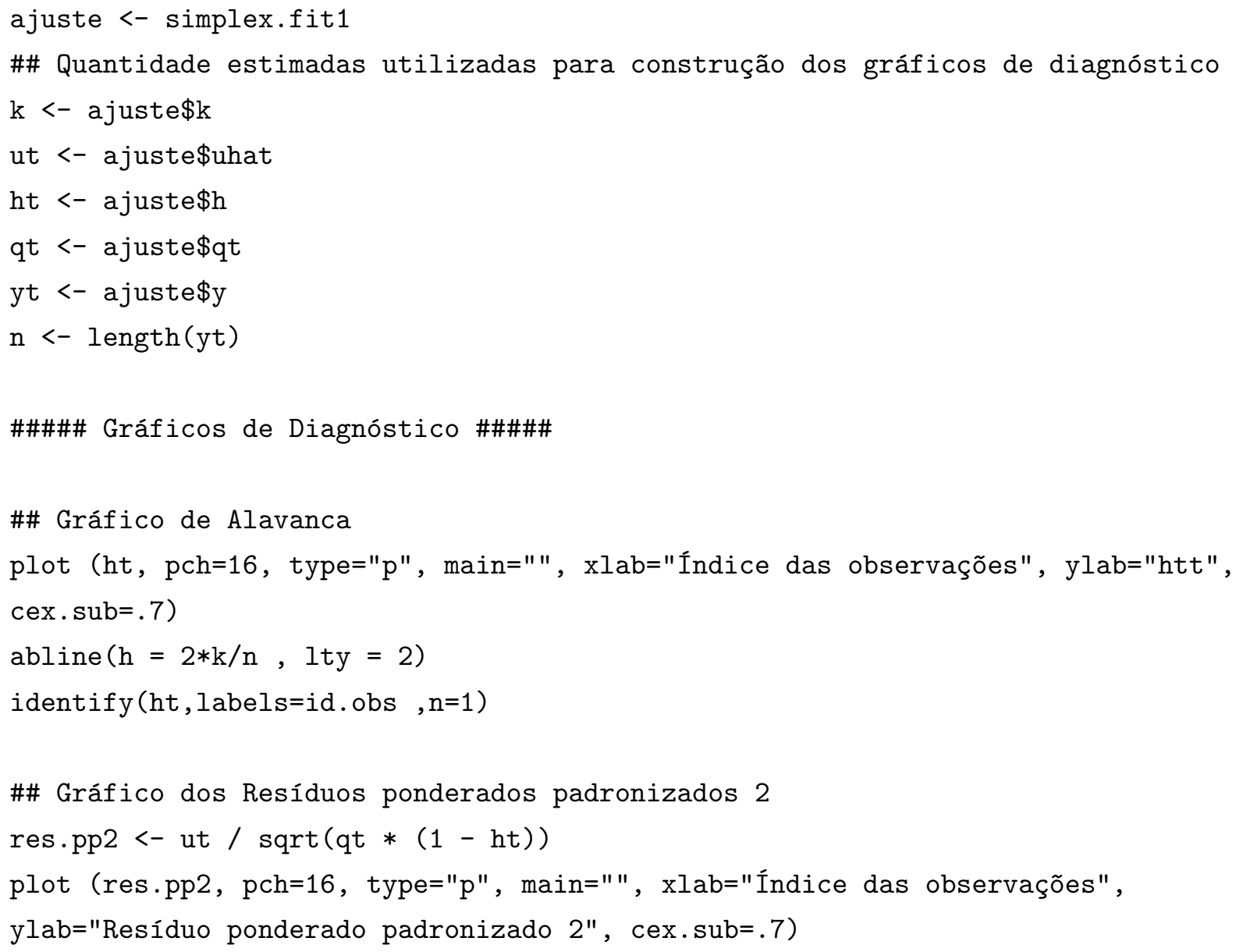




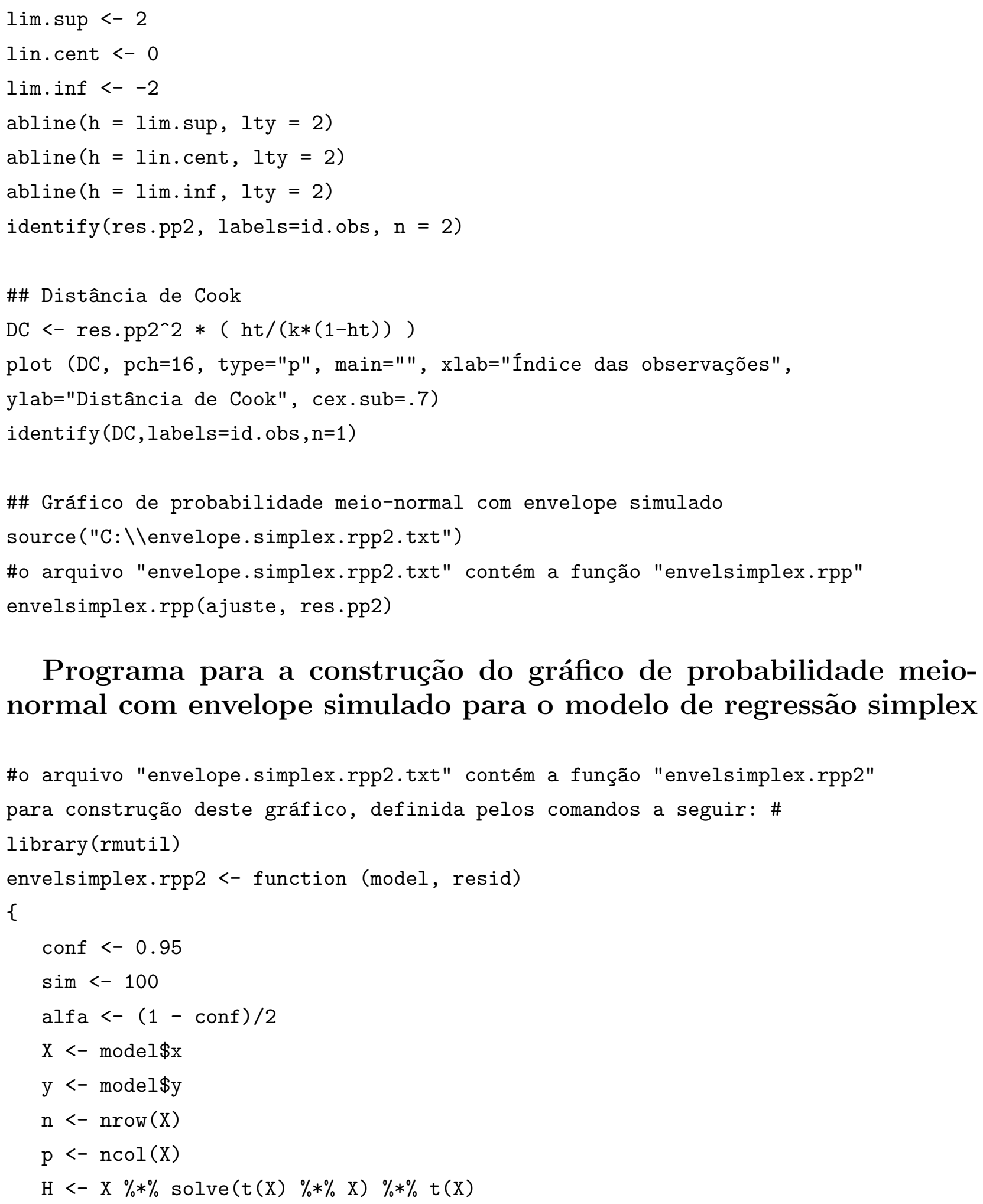

Programa para a construção do gráfico de probabilidade meionormal com envelope simulado para o modelo de regressão simplex 


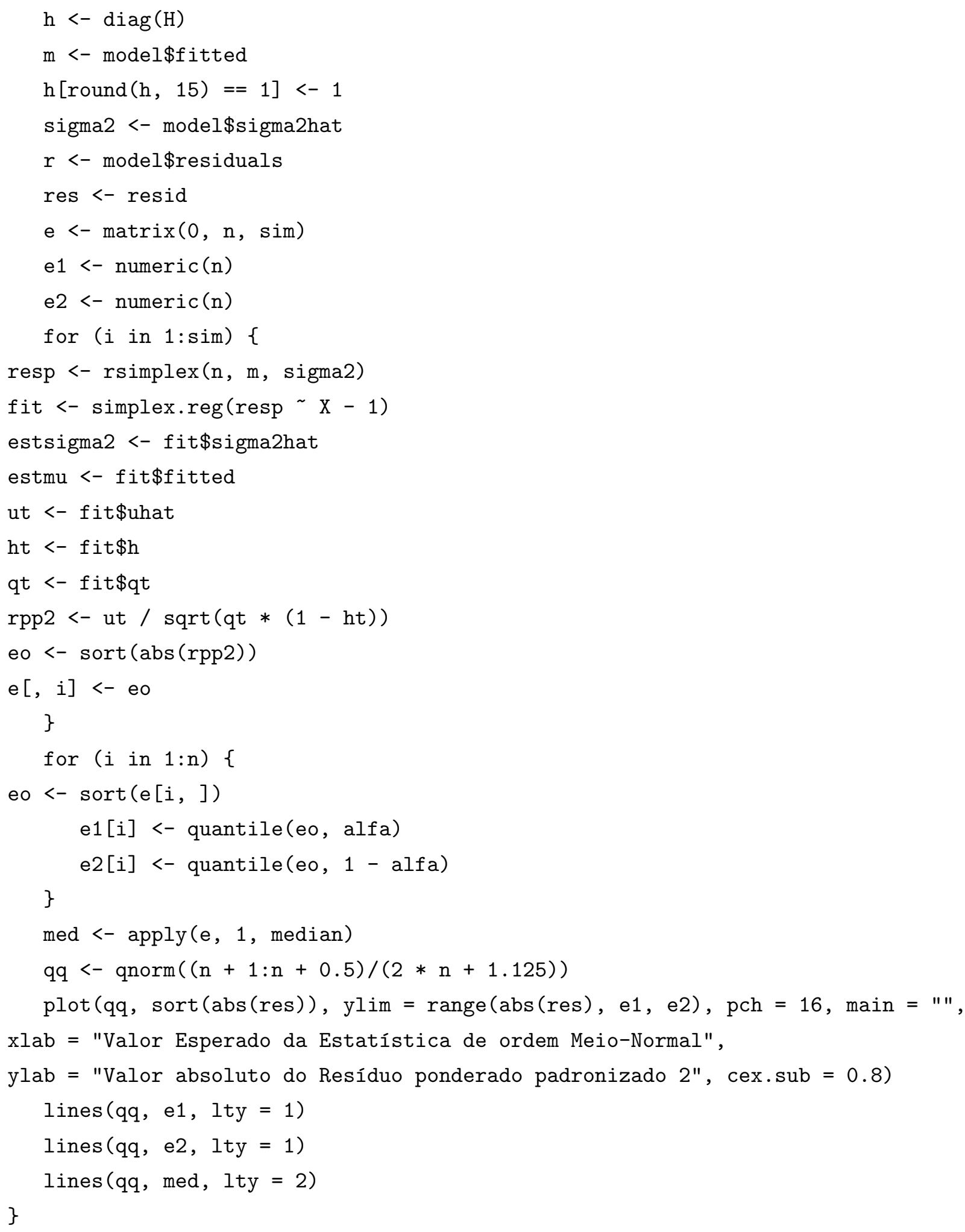




\section{Referências Bibliográficas}

Andrade, A. C. G. (2007), Efeitos de especificação incorreta da função de ligação no modelo de regressão beta, Dissertação de Mestrado, IME - USP, São Paulo.

Barndorff-Nielsen, O. E. \& Jørgensen, B. (1991), 'Some parametric models on the simplex', Journal of Multivariate Analysis 39, 106-116.

Brownlee, K. A. (1965), Statistical Theory and Methodology in Science and Engineering, John Wiley and Sons, London. 2.ed.

Cook, R. D. (1977), 'Detection of influential observations in linear regressions', Technometrics 19, $15-18$.

Cox, C. (1996), 'Nonlinear quasi-likelihood models: applications to continuous proportions', Computational Statistics \& Data Analysis 21, 449-61.

Espinheira, P. L., Ferrari, S. L. P. \& Cribari-Neto, F. (2008), 'On beta regression residuals', Journal of Applied Statistics. A aparecer.

Ferrari, S. L. P. \& Cribari-Neto, F. (2004), 'Beta regression for modelling rates and proportions', Journal of Applied Statistics 31, 799-815.

Jørgensen, B. (1997), The theory of dispersion models, Chapman and Hall, London.

Kieschnick, R. \& McCullough, B. D. (2003), 'Regression analysis of variates observed on (0, 1): percentages, proportions and fraction', Statistical Modelling 3, 193-213.

Lindsey, J. (2007), 'rmutil: Utilities for Nonlinear Regression and Repeated Measurements Models', popgen.unimaas.nl/ jlindsey/rcode.html. R package version 1.0.

McCullagh, P. \& Nelder, J. A. (1989), Generalized Linear Models, Chapman and Hall, London. 2.ed.

Neter, J., Kutner, M. H., Naschtheim, C. J. \& Wasserman, W. (1996), Appplied Linear Statistical Models, McGraw Hill, Chicago. 4.ed. 
Nocedal, J. \& Wright, S. J. (1999), Numerical optimization, Spriger-Verlag, New York.

Oliveira, M. S. (2004), Um modelo de regressão beta: teoria e aplicações, Dissertação de Mestrado, IME - USP, São Paulo.

Papke, L. \& Wooldridge, J. (1996), 'Econometric methods for frational response variables with an application to 401(k) plan participation rates', Journal of Applied Econometrics 11, 619-32.

Paula, G. A. (2004), 'Modelos de regressão com apoio computacional, Notas de aulas', http:www//ime.usp.br/ giapaula.

Prater, N. H. (1956), 'Estimate gasoline yields from crude', Petroleum Refiner 35, 236-238.

R Development Core Team (2007), 'R: A language and environment for statistical computing', http://www.R-project.org. ISBN 3-900051-07-0.

Rao, C. R. (1973), Linear Statistical Inference and Its Applications, Wiley, New York. 2.ed.

Sen, P. \& Singer, J. M. (1993), Large Sample Methods in Statistics: An Introduction With Applications, Chapmann and Hall, New York.

Simas, A. B. (2006), Beta regression for modelling rates and proportions. R package version 1.2 .

Song, P. X.-K. \& Tan, M. (2000), 'Marginal models for longitudinal continuous proportional data', Biometrics 56, 496-502.

Venezuela, M. K., Botter, D. A. \& Sandoval, M. C. (2007), 'Diagnostics techniques in generalized estimating equations', Journal of Statistical Computation and Simulation 77, 879888. 